Pacific

Journal of

Mathematics

THE DECOMPOSITION OF GLOBAL CONFORMAL INVARIANTS: SOME TECHNICAL PROOFS II

SPYROS ALEXAKIS 


\title{
THE DECOMPOSITION OF GLOBAL CONFORMAL INVARIANTS: SOME TECHNICAL PROOFS II
}

\author{
SPYROS ALEXAKIS
}

This paper complements our research monograph The decomposition of global conformal invariants (Princeton University Press, 2012) in proving a conjecture of Deser and Schwimmer regarding the algebraic structure of "global conformal invariants"; these are defined to be conformally invariant integrals of geometric scalars. The conjecture asserts that the integrand of any such integral can be expressed as a linear combination of a local conformal invariant, a divergence and of the Chern-Gauss-Bonnet integrand.

The present paper provides a proof of certain purely algebraic statements announced in our previous work and whose rather technical proof was deferred to this paper; the lemmas proven here serve to reduce "main algebraic propositions" to certain technical inductive statements.

1. Introduction 1

2. Proof of the technical lemmas from [A 2010] 8

2A. Restatement of the technical Lemmas 4.6-4.9 from [A 2010] 8

2B. Two more technical lemmas 13

2C. Proof of Lemmas 4.6, 4.8 in [A 2010]: The main part 29

2D. The remaining cases of Lemma $2.9 \quad 46$

2E. Proof of Lemmas 2.2 and $2.4 \quad 72$

3. The proof of Proposition 1.1 in the special cases 76

3A. The direct proof of Proposition 1.1 (in Case II) in the special cases 76

3B. The remaining cases of Proposition 1.1 in Case III 84

Acknowledgement $\quad 86$

References $\quad 86$

MSC2010: primary 53A55, 53B20; secondary 53A30.

Keywords: conformal invariant, global conformal invariant, Deser-Schwimmer conjecture,

Riemannian invariant. 


\section{Introduction}

This paper complements our earlier work [A 2010, 2011, 2012] in proving a conjecture of Deser and Schwimmer [1993] regarding the algebraic structure of "global conformal invariants". It provides a (rather technical) proof of certain lemmas announced in [A 2010, 2012].

We recall that a global conformal invariant is an integral of a natural scalarvalued function of Riemannian metrics, $\int_{M^{n}} P(g) d V_{g}$, which remains invariant under conformal rescalings of the underlying metric. ${ }^{1}$ More precisely, $P(g)$ is assumed to be a linear combination, $P(g)=\sum_{l \in L} a_{l} C^{l}(g)$, where each $C^{l}(g)$ is a complete contraction in the form

$$
\operatorname{contr}^{l}\left(\nabla^{\left(m_{1}\right)} R \otimes \cdots \otimes \nabla^{\left(m_{s}\right)} R\right) ;
$$

here each factor $\nabla^{(m)} R$ stands for the $m^{\text {th }}$ iterated covariant derivative of the curvature tensor $R, \nabla$ is the Levi-Civita connection of the metric $g$ and $R$ is the curvature associated to this connection. The contractions are taken with respect to the quadratic form $g^{i j}$. In the present paper, along with [A 2011, 2012], we prove: Theorem. Assume that $P(g)=\sum_{l \in L} a_{l} C^{l}(g)$, where each $C^{l}(g)$ is a complete contraction in the form (1-1), with weight $-n$. Assume that for every closed Riemannian manifold $\left(M^{n}, g\right)$ and every $\phi \in C^{\infty}\left(M^{n}\right)$,

$$
\int_{M^{n}} P\left(e^{2 \phi} g\right) d V_{e^{2 \phi} g}=\int_{M^{n}} P(g) d V_{g} .
$$

We claim that $P(g)$ can then be expressed in the form

$$
P(g)=W(g)+\operatorname{div}_{i} T^{i}(g)+\operatorname{Pfaff}\left(R_{i j k l}\right) .
$$

Here $W(g)$ stands for a local conformal invariant of weight $-n$ (meaning that $W\left(e^{2 \phi} g\right)=e^{-n \phi} W(g)$ for every $\left.\phi \in C^{\infty}\left(M^{n}\right)\right), \operatorname{div}_{i} T^{i}(g)$ is the divergence of $a$ Riemannian vector field of weight $-n+1$, and $\operatorname{Pfaff}\left(R_{i j k l}\right)$ is the Pfaffian of the curvature tensor.

Before we discuss the position of the present paper in the entire work [A 2010, 2011, 2012] we digress to describe the relation between the present series of papers with classical and recent work on scalar local invariants in various geometries.

Broad discussion. The theory of local invariants of Riemannian structures (and indeed, of more general geometries, such as conformal, projective, or CR) has a long history. As discussed in [A 2012], the original foundations of this field were laid in the work of Hermann Weyl and Élie Cartan; see [Weyl 1939; Cartan 1896]. The task of writing out local invariants of a given geometry is intimately connected

\footnotetext{
${ }^{1}$ See the introduction of [A 2012] for a detailed discussion of the Deser-Schwimmer conjecture, and for background on scalar Riemannian invariants.
} 
with understanding polynomials in a space of tensors with given symmetries; these polynomials are required to remain invariant under the action of a Lie group on the components of the tensors. In particular, the problem of writing down all local Riemannian invariants reduces to understanding the invariants of the orthogonal group.

In more recent times, a major program was laid out by C. Fefferman [1976] aimed at finding all scalar local invariants in CR geometry. This was motivated by the problem of understanding the local invariants that appear in the asymptotic expansion of the Bergman and Szegó kernels of strictly pseudoconvex CR manifolds, in a similar way to Riemannian invariants that appear in the asymptotic expansion of the heat kernel; the study of the local invariants in the singularities of these kernels led to important breakthroughs in [Bailey et al. 1994b] and more recently by Hirachi [2000]. This program was later extended to conformal geometry in [Fefferman and Graham 1985]. Both these geometries belong to a broader class of structures, the parabolic geometries; these admit a principal bundle whose structure group is a parabolic subgroup $P$ of a semisimple Lie group $G$, and a Cartan connection on that principle bundle (see the introduction in [Čap and Gover 2002]). An important question in the study of these structures is the problem of constructing all their local invariants, which can be thought of as the natural, intrinsic scalars of these structures.

In the context of conformal geometry, the first (modern) landmark in understanding local conformal invariants was the work of Fefferman and Graham [1985], where they introduced the ambient metric. This allows one to construct local conformal invariants of any order in odd dimensions, and up to order $\frac{n}{2}$ in even dimensions. The question is then whether all invariants arise via this construction.

The subsequent work of Bailey-Eastwood-Graham [1994b] proved that this is indeed true in odd dimensions; in even dimensions, they proved that the result holds when the weight (in absolute value) is bounded by the dimension. The ambient metric construction in even dimensions was recently extended by Graham and Hirachi [2008]; this enables them to identify in a satisfactory way all local conformal invariants, even when the weight (in absolute value) exceeds the dimension.

An alternative construction of local conformal invariants can be obtained via the tractor calculus introduced by Bailey et al. [1994a]. This construction bears a strong resemblance to the Cartan conformal connection, and to the work of T.Y. Thomas [1934]. The tractor calculus has proven to be very universal; tractor bundles have been constructed [Čap and Gover 2002] for an entire class of parabolic geometries. The relation between the conformal tractor calculus and the Fefferman-Graham ambient metric has been elucidated in [Čap and Gover 2003]. 
The present paper, along with [A 2010, 2011, 2012], while pertaining to the question above (given that it ultimately deals with the algebraic form of local Riemannian and conformal invariants), nonetheless addresses a different type of problem: We here consider Riemannian invariants $P(g)$ for which the integral $\int_{M^{n}} P(g) d V_{g}$ remains invariant under conformal changes of the underlying metric; we then seek to understand the possible algebraic form of the integrand $P(g)$, ultimately proving that it can be decomposed in the way that Deser and Schwimmer asserted. It is thus not surprising that the prior work on the construction and understanding of local conformal invariants, in [A 2011] and in the second chapter of [A 2012], plays a central role in this endeavor.

On the other hand, a central element of our proof are the "Main algebraic propositions" 2.28, 3.27, 3.28 in [A 2012]; these deal exclusively with algebraic properties of the classical scalar Riemannian invariants. (These "main algebraic propositions" are discussed in brief below. A generalization of these propositions is the Proposition 1.1 below). The "Fundamental proposition 1.1" makes no reference to integration; it is purely a statement concerning local Riemannian invariants. Thus, while the author was led to led to the main algebraic propositions in [A 2012] out of the strategy that he felt was necessary to solve the Deser-Schwimmer conjecture, they can be thought of as results of an independent interest. The proof of these propositions, presented in the second part of [A 2012] (and certain claims announced there proven in the present paper), is in fact not particularly intuitive. It is the author's sincere hope that deeper insight (and hopefully a more intuitive proof) will be obtained in the future as to why these algebraic propositions hold.

Let us now discuss the position of the present paper in the entire work [A 2010, 2011, 2012] in more detail: In the first part of [A 2012] (complemented by [A 2011]) we proved that the Deser-Schwimmer conjecture holds, provided one can show certain "main algebraic propositions," announced in Chapters 2 and 3 in [A 2012]. In [A 2010] (which is reproduced in Chapter 4 of [A 2012] — for convenience we refer to propositions and lemmas in [A 2010]; the same propositions can be found in [A 2012] with different numbering) we claimed a more general proposition which implies the "main algebraic propositions;" this new "Fundamental proposition" 2.1 in [A 2010] $]^{2}$ is to be proven by an induction of four parameters. In [A 2010] we also reduced the inductive step of Proposition 2.1 to three lemmas (in particular we distinguished Cases I, II, III of Proposition 2.1 by examining the tensor fields appearing in its hypothesis, see (1-7) below; Lemmas 3.1, 3.2, 3.5 in [A 2010 $^{3}$ correspond to these three cases). We proved that these three lemmas imply the inductive step of the Fundamental proposition in Cases I, II, III respectively, apart from certain special cases which were deferred to the present

\footnotetext{
${ }^{2}$ This is reproduced as Proposition 4.13 in [A 2012].

${ }^{3}$ These correspond respectively to Lemmas 4.16, 4.19 and 4.24 in [A 2012].
} 
paper. In these special cases we will derive Proposition 2.1 in [A 2010] directly, ${ }^{4}$ in Section 3. Now, in proving that the inductive step of Proposition 1.1 follows from Lemmas 3.1, 3.2, 3.5 in [A 2010] we asserted certain technical lemmas, whose proof was deferred to the present paper. These were Lemmas 4.6, 4.8, and 4.7, 4.9 in [A 2010]; ${ }^{5}$ also, the proof of Lemma A.1 in [A 2010] was deferred to the present paper. We prove all these lemmas from [A 2010] in Section 2.

For reference purposes, and for the reader's convenience, we recall the precise formulation of the "Fundamental proposition" 2.1 in [A 2010], referring the reader to [A 2010] for a definition of many of the terms appearing in the formulation. First however, we will recall (schematically) the first "Main algebraic proposition" 2.28 in [A 2012]; this is a special case of Proposition 2.1 in [A 2010], and provides a simpler version of it.

A simpler version of Proposition 2.1 in [A 2010]. Given a Riemannian metric $g$ over an $n$-dimensional manifold $M^{n}$ and auxiliary $C^{\infty}$ scalar-valued functions $\Omega_{1}, \ldots, \Omega_{p}$ defined over $M^{n}$, the objects of study are linear combinations of tensor fields $\sum_{l \in L} a_{l} C_{g}^{l, i_{1} \ldots i_{\alpha}}$, where each $C_{g}^{l, i_{1} \ldots i_{\alpha}}$ is a partial contraction with $\alpha$ free indices, in the form

$$
\operatorname{pcontr}\left(\nabla^{(m)} R \otimes \cdots \otimes \nabla^{\left(m_{s}\right)} R \otimes \nabla^{\left(b_{1}\right)} \Omega_{1} \otimes \cdots \otimes \nabla^{\left(b_{m}\right)} \Omega_{p}\right) ;
$$

here $\nabla^{(m)} R$ stands for the $m^{t h}$ covariant derivative of the curvature tensor $R,{ }^{6}$ and $\nabla^{(b)} \Omega_{h}$ stands for the $b^{\text {th }}$ covariant derivative of the function $\Omega_{h}$. A partial contraction means that we have list of pairs of indices $(a, b), \ldots,(c, d)$ in (1-2), that are contracted against each other via the metric $g^{i j}$. The remaining indices (which are not contracted against another index in (1-2)) are the free indices $i_{1}, \ldots, i_{\alpha}$.

The "Main algebraic proposition" 2.28 in [A 2012] (roughly) asserts the following: Let $\sum_{l \in L_{\mu}} a_{l} C_{g}^{l, i_{1} \ldots i_{\mu}}$ stand for a linear combination of partial contractions in the form (1-2), where each $C_{g}^{l, i_{1} \ldots i_{\mu}}$ has a given number $\sigma_{1}$ of factors and a given number $p$ of factor $\nabla^{(b)} \Omega_{h}$. Assume also that $\sigma_{1}+p \geq 3$, each $b_{i} \geq 2{ }^{7}$ and that for each contracting pair of indices $(a, b)$ in any given $C_{g}^{l, i_{1} \ldots i_{\mu}}$, the indices ${ }_{a},{ }_{b}$ do not belong to the same factor. Assume also the rank $\mu>0$ is fixed and each partial contraction $C_{g}^{l, i_{1} \ldots i_{\mu}}, l \in L_{\mu}$ has a given weight $-n+\mu .{ }^{8}$ Let also $\sum_{l \in L_{>\mu}} a_{l} C_{g}^{l, i_{1} \ldots i_{y_{l}}}$ stand for a (formal) linear combination of partial contractions of weight $-n+y_{l}$, with all the properties of the terms indexed in $L_{\mu}$, except that now all the partial contractions have a different rank $y_{l}$, and each $y_{l}>\mu$.

\footnotetext{
${ }^{4}$ By this we mean without recourse to the Lemmas 3.1, 3.2, 3.5 in [A 2010].

${ }^{5}$ These correspond to Lemmas 4.35, 4.41, 4.37 and 4.42 in [A 2012].

${ }^{6}$ In particular it is a tensor of rank $m+4$; if we write out its free indices it would be in the form $\nabla_{r_{1} \ldots r_{m}}^{(m)} R_{i j k l}$.

${ }^{7} b_{i} \geq 2$ means that the function $\Omega_{i}$ is differentiated at least twice.

${ }^{8}$ See [A 2012] for a precise definition of weight.
} 
Assume also that the local equation

$$
\sum_{l \in L_{\mu}} a_{l} X \operatorname{div}_{i_{1}} \ldots X \operatorname{div}_{i_{\mu}} C_{g}^{l, i_{1} \ldots i_{\mu}}+\sum_{l \in L_{>\mu}} a_{l} X \operatorname{div}_{i_{1}} \ldots X \operatorname{div}_{i_{y_{l}}} C_{g}^{l, i_{1} \ldots i_{y_{l}}}=0
$$

holds modulo complete contractions with $\sigma+1$ factors. Here, given a partial contraction $C_{g}^{l, i_{1} \ldots i_{\alpha}}$ in the form (1-2) $X \operatorname{div}_{i_{s}}\left[C_{g}^{l, i_{1} \ldots i_{\alpha}}\right]$ stands for sum of $\sigma-1$ terms in $\operatorname{div}_{i_{s}}\left[C_{g}^{l, i_{1} \ldots i_{\alpha}}\right]$ where the derivative $\nabla^{i_{s}}$ is not allowed to hit the factor to which the free index $i_{s}$ belongs. ${ }^{9}$

The "Main algebraic proposition" 2.28 in [A 2012] says that there exists a linear combination of partial contractions in the form (1-2), $\sum_{h \in H} a_{h} C_{g}^{h, i_{1} \ldots i_{\mu+1}}$, with all the properties of the terms indexed in $L_{>\mu}$, and all with rank $(\mu+1)$, so that

$$
\sum_{l \in L_{1}} a_{l} C_{g}^{l,\left(i_{1} \ldots i_{\mu}\right)}+\sum_{h \in H} a_{h} X \operatorname{div}_{i_{\mu+1}} C_{g}^{l,\left(i_{1} \ldots i_{\mu}\right) i_{\mu+1}}=0
$$

the above holds modulo terms of length $\sigma+1$. The symbol (..) means that we are symmetrizing over the indices between parentheses.

In [A 2010] we set up a multiple induction by which we will prove the "main algebraic propositions" in Chapters 2, 3 in [A 2012]. The inductive step is proven in the "Fundamental proposition" 2.1 in [A 2010], which we reproduce here in Proposition 1.1. This deals with tensor fields in the forms

$$
\begin{aligned}
& \operatorname{pcontr}\left(\nabla^{\left(m_{1}\right)} R_{i j k l} \otimes \cdots \otimes \nabla^{\left(m_{s}\right)} R_{i j k l} \otimes \nabla^{\left(b_{1}\right)} \Omega_{1}\right. \\
& \left.\quad \otimes \cdots \otimes \nabla^{\left(b_{p}\right)} \Omega_{p} \otimes \nabla \phi_{1} \otimes \cdots \otimes \nabla \phi_{u}\right) \\
& \operatorname{pcontr}\left(\nabla^{\left(m_{1}\right)} R_{i j k l} \otimes \cdots \otimes \nabla^{\left(m_{\sigma_{1}}\right)} R_{i j k l}\right. \\
& \quad \otimes S_{*} \nabla^{\left(v_{1}\right)} R_{i j k l} \otimes \cdots \otimes S_{*} \nabla^{\left(v_{t}\right)} R_{i j k l} \\
& \otimes \nabla^{\left(b_{1}\right)} \Omega_{1} \otimes \cdots \otimes \nabla^{\left(b_{p}\right)} \Omega_{p} \otimes \nabla \phi_{z_{1}} \otimes \cdots \otimes \nabla \phi_{z_{w}} \\
& \left.\otimes \nabla \phi_{z_{w+1}^{\prime}}^{\prime} \otimes \cdots \otimes \nabla \phi_{z_{w+d}^{\prime}}^{\prime} \otimes \cdots \otimes \nabla \tilde{\phi}_{z_{w+d+1}} \otimes \cdots \otimes \nabla \tilde{\phi}_{z_{w+d+y}}\right) .
\end{aligned}
$$

(See the introduction in [A 2010] for a detailed description of the above form.) In keeping with the conventions introduced in [A 2010], we remark that a complete or partial contraction in the above form will be called "acceptable" if each $b_{i} \geq 2$, for $1 \leq i \leq p .^{10}$

\footnotetext{
${ }^{9}$ Recall that given a partial contraction $C_{g}^{l, i_{1} \ldots i_{\alpha}}$ in the form (1-2) with $\sigma$ factors, $\operatorname{div}_{i_{s}} C_{g}^{l, i_{1} \ldots i_{\alpha}}$ is a sum of $\sigma$ partial contractions of rank $\alpha-1$. The first summand arises by adding a derivative $\nabla^{i_{s}}$ onto the first factor $T_{1}$ and then contracting the upper index ${ }^{i_{s}}$ against the free index $i_{s}$; the second summand arises by adding a derivative $\nabla^{i_{s}}$ onto the second factor $T_{2}$ and then contracting the upper index $i_{s}$ against the free index $i_{s}$ and so on.

${ }^{10}$ In other words, we are requiring each function $\Omega_{i}$ is differentiated at least twice.
} 
Proposition 1.1. Consider two linear combinations of acceptable tensor fields in the form (1-6),

$$
\begin{gathered}
\sum_{l \in L_{\mu}} a_{l} C_{g}^{l, i_{1} \ldots i_{\mu}}\left(\Omega_{1}, \ldots, \Omega_{p}, \phi_{1}, \ldots, \phi_{u}\right), \\
\sum_{l \in L_{>\mu}} a_{l} C_{g}^{l, i_{1} \ldots i_{\beta_{l}}}\left(\Omega_{1}, \ldots, \Omega_{p}, \phi_{1}, \ldots, \phi_{u}\right),
\end{gathered}
$$

where each tensor field above has real length $\sigma \geq 3$ and a given simple character $\vec{\kappa}_{\text {simp. }}$. We assume that for each $l \in L_{>\mu}, \beta_{l} \geq \mu+1$. We also assume that none of the tensor fields of maximal refined double character in $L_{\mu}$ are "forbidden" (see Definition 2.12 in [A 2010]).

We denote by

$$
\sum_{j \in J} a_{j} C_{g}^{j}\left(\Omega_{1}, \ldots, \Omega_{p}, \phi_{1}, \ldots, \phi_{u}\right)
$$

a generic linear combination of complete contractions (not necessarily acceptable) in the form (1-5) that are simply subsequent to $\vec{\kappa}_{\text {simp. }}{ }^{11}$ We assume that

$$
\begin{gathered}
\sum_{l \in L_{\mu}} a_{l} X \operatorname{div}_{i_{1}} \ldots X \operatorname{div}_{i_{\alpha}} \\
\quad \times C_{g}^{l, i_{1} \ldots i_{\mu}}\left(\Omega_{1}, \ldots, \Omega_{p}, \phi_{1}, \ldots, \phi_{u}\right) \\
+\sum_{l \in L_{>\mu}} a_{l} X \operatorname{div}_{i_{1}} \ldots X \operatorname{div}_{i_{\beta_{l}}} \\
\quad \times C_{g}^{l, i_{1} \ldots i_{\beta_{l}}}\left(\Omega_{1}, \ldots, \Omega_{p}, \phi_{1}, \ldots, \phi_{u}\right) \\
+\sum_{j \in J} a_{j} C_{g}^{j}\left(\Omega_{1}, \ldots, \Omega_{p}, \phi_{1}, \ldots, \phi_{u}\right)=0 .
\end{gathered}
$$

We draw our conclusion with a little more notation: We break the index set $L_{\mu}$ into subsets $L^{z}, z \in Z$ ( $Z$ is finite), with the rule that each $L^{z}$ indexes tensor fields with the same refined double character, and conversely two tensor fields with the same refined double character must be indexed in the same $L^{z}$. For each index set $L^{z}$, we denote the refined double character in question by $\vec{L}^{z}$. Consider the subsets $L^{z}$ that index the tensor fields of maximal refined double character. ${ }^{12}$ We assume that the index set of those $z$ is $Z_{\text {Max }} \subset Z$.

We claim that for each $z \in Z_{\text {Max }}$ there is some linear combination of acceptable $(\mu+1)$-tensor fields,

\footnotetext{
${ }^{11}$ Of course if $\operatorname{Def}\left(\vec{\kappa}_{\text {simp }}\right)=\varnothing$ then by definition $\sum_{j \in J} \cdots=0$.
}

${ }^{12}$ Note that in any set $S$ of $\mu$-refined double characters with the same simple character there is going to be a subset $S^{\prime}$ consisting of the maximal refined double characters. 


$$
\sum_{r \in R^{z}} a_{r} C_{g}^{r, i_{1} \ldots i_{\alpha+1}}\left(\Omega_{1}, \ldots, \Omega_{p}, \phi_{1}, \ldots, \phi_{u}\right)
$$

where each

$$
C_{g}^{r, i_{1} \ldots i_{\mu+1}}\left(\Omega_{1}, \ldots, \Omega_{p}, \phi_{1}, \ldots, \phi_{u}\right)
$$

has a $\mu$-double character $\vec{L}_{1}^{z}$ and also the same set of factors $S_{*} \nabla^{(v)} R_{i j k l}$ as in $\vec{L}^{z}$ contain special free indices, so that

$$
\begin{aligned}
& \sum_{l \in L^{z}} a_{l} C_{g}^{l, i_{1} \ldots i_{\mu}}\left(\Omega_{1}, \ldots, \Omega_{p}, \phi_{1}, \ldots, \phi_{u}\right) \nabla_{i_{1}} v \ldots \nabla_{i_{\mu}} v \\
& -\sum_{r \in R^{z}} a_{r} X \operatorname{div}_{i_{\mu+1}} C_{g}^{r, i_{1} \ldots i_{\mu+1}}\left(\Omega_{1}, \ldots, \Omega_{p}, \phi_{1}, \ldots, \phi_{u}\right) \nabla_{i_{1}} v \ldots \nabla_{i_{\mu}} v \\
& \quad=\sum_{t \in T_{1}} a_{t} C_{g}^{t, i_{1} \ldots i_{\mu}}\left(\Omega_{1}, \ldots, \Omega_{p},, \phi_{1}, \ldots, \phi_{u}\right) \nabla_{i_{1}} v \ldots \nabla_{i_{\mu}} v
\end{aligned}
$$

modulo complete contractions of length $\geq \sigma+u+\mu+1$. Here each

$$
C_{g}^{t, i_{1} \ldots i_{\mu}}\left(\Omega_{1}, \ldots, \Omega_{p}, \phi_{1}, \ldots, \phi_{u}\right)
$$

is acceptable and is either simply or doubly subsequent to $\vec{L}^{z}{ }^{13}$

(See the first section in [A 2010] for a description of the notions of real length, acceptable tensor fields, simple character, refined double character, maximal refined double character, simply subsequent, strongly doubly subsequent.) We prove Proposition 1.1 by a multiple induction on the parameters $-n$ (the weight of the complete contractions appearing in (1-7)), $\sigma$ (the total number of factors in the form $\nabla^{(m)} R_{i j k l}, S_{*} \nabla^{(v)} R_{i j k l}, \nabla^{(A)} \Omega_{h}$ among the partial contractions in (1-7)), ${ }^{14} \Phi$ (the number of factors $\nabla \phi_{1}, \ldots, \nabla \phi_{u}$ appearing in (1-7)), and $\sigma_{1}+\sigma_{2}$ (the total number of factors $\left.\nabla^{(m)} R_{i j k l}, S_{*} \nabla^{(v)} R_{i j k l}\right)$. When $\Phi=0$, Proposition 1.1 coincides with the "Main algebraic proposition" 2.28 in [A 2012] outlined above. ${ }^{15}$

\section{Proof of the technical lemmas from [A 2010]}

2A. Restatement of the technical Lemmas 4.6-4.9 from [A 2010]. We start by recalling a definition from [A 2010] that will be used frequently in the present paper:

${ }^{13}$ Recall that "simply subsequent" means that the simple character of $C_{g}^{t, i_{1} \ldots i_{\mu}}$ is subsequent to $\operatorname{Simp}\left(\vec{L}^{z}\right)$.

${ }^{14}$ The partial contractions in (1-7) are assumed to all have the same simple character-this implies that they all have the same number of factors $\nabla^{(m)} R_{i j k l}, S_{*} \nabla^{(v)} R_{i j k l}, \nabla^{(A)} \Omega_{h}$ respectively.

${ }^{15}$ Similarly, the "Main algebraic propositions" 3.27 and 3.28 in Chapter 3 of [A 2012] coincide with Proposition 1.1 above when $\Phi=1$. 
Definition. Consider any partial contraction in the form (1-6). We consider any set of indices, $\left\{x_{1}, \ldots, x_{s}\right\}$ belonging to a factor $T$, which is either in the form $\nabla^{(B)} \Omega_{h}$ or $\nabla^{(m)} R_{i j k l}$. We assume that these indices are not free and are not contracting against a factor $\nabla \phi_{h}$.

If the indices belong to a factor $T$ in the form $\nabla^{(B)} \Omega_{h}$ then $\left\{x_{1}, \ldots, x_{s}\right\}$ are removable provided $B \geq s+2$.

Now, we consider indices that belong to a factor $T$ in the form $\nabla^{(m)} R_{i j k l}$ (and are not free and do not contract against a factor $\nabla \phi_{h}$ ). Any such index $x$ which is a derivative index will be removable. Furthermore, if $T$ has at least two free derivative indices, then if neither of the indices ${ }_{i},{ }_{j}$ are free we will say one of $i, j$ is removable; accordingly, if neither of ${ }_{k}, l$ is free then we will say that one of ${ }_{k}, l$ is removable. Moreover, if $T$ has one free derivative index then: if none of the indices ${ }_{i},{ }_{j}$ are free we will say that one of the indices ${ }_{i},{ }_{j}$ is removable; on the other hand if one of the indices ${ }_{i},{ }_{j}$ is also free and none of the indices ${ }_{k}, l$ are free then we will say that one of the indices ${ }_{k}, l$ is removable.

Now, we consider a set of indices $\left\{x_{1}, \ldots, x_{s}\right\}$ that belong to a factor $T$ in the form $S_{*} \nabla_{r_{1} \ldots r_{\nu}}^{(v)} R_{i j k l}$; if $\left\{x_{1}, \ldots, x_{s}\right\} \subset\left\{r_{1} \ldots r_{\nu}, j\right\}$ and none of them are free and none of them contract against a factor $\nabla \phi_{x}$, then we will say this set of indices is removable. Furthermore, we will say that the indices $k, l$ in such a factor are removable if neither $_{k}$ nor ${ }_{l}$ is free and $v>0$ and at least one of the indices $r_{1}, \ldots, r_{v},{ }_{j}$ is free.

For the two Lemmas 2.1 and 2.2 we will consider tensor fields in the form

$$
\begin{aligned}
\operatorname{pcontr}( & \nabla^{\left(m_{1}\right)} R_{i j k l} \otimes \cdots \otimes \nabla^{\left(m_{\sigma_{1}}\right)} R_{i j k l} \\
& \otimes S_{*} \nabla^{\left(v_{1}\right)} R_{i j k l} \otimes \cdots \otimes S_{*} \nabla^{\left(v_{t}\right)} R_{i j k l} \otimes \nabla Y \\
& \otimes \nabla^{\left(b_{1}\right)} \Omega_{1} \otimes \cdots \otimes \nabla^{\left(b_{p}\right)} \Omega_{p} \otimes \nabla \phi_{z_{1}} \cdots \otimes \nabla \phi_{z_{w}} \\
& \left.\otimes \nabla \phi_{z_{w+1}^{\prime}}^{\prime} \otimes \cdots \otimes \nabla \phi_{z_{w+d}}^{\prime} \otimes \cdots \otimes \nabla \tilde{\phi}_{z_{w+d+1}} \otimes \cdots \otimes \nabla \tilde{\phi}_{z_{w+d+y}}\right) .
\end{aligned}
$$

(Notice this is the same as the form (1-6), but for the fact that we have inserted a factor $\nabla Y$ in the second line.) We recall that for a partial contraction $C_{g}^{i_{1} \ldots i_{h}}$ in the above form, $X_{*} \operatorname{div}_{i_{r}} C_{g}^{i_{1} \ldots i_{h}}$ stands for the sublinear combination in the divergence $\operatorname{div}_{i_{r}} C_{g}^{i_{1} \ldots i_{h}}$ where the derivative $\nabla^{i_{r}}$ is not allowed to hit the factor to which $i_{r}$ belongs, nor any factor $\nabla \phi_{x}$, nor the factor $\nabla Y$.

The claims whose proof was deferred to the present paper are then as follows:

Lemma 2.1. Assume that

$$
\begin{aligned}
\sum_{h \in H_{2}} a_{h} X_{*} \operatorname{div}_{i_{1}} \ldots X_{*} \operatorname{div}_{i_{a_{h}}} C_{g}^{h, i_{1} \ldots i_{a_{h}}}\left(\Omega_{1}, \ldots, \Omega_{p}, Y, \phi_{1}, \ldots, \phi_{u^{\prime}}\right) \\
=\sum_{j \in J} a_{j} C_{g}^{j}\left(\Omega_{1}, \ldots, \Omega_{p}, \phi_{1}, \ldots, \phi_{u^{\prime}}\right),
\end{aligned}
$$


where all tensor fields have rank $a_{h} \geq \alpha$. All tensor fields have a given u-simple character $\vec{\kappa}_{\text {simp }}^{\prime}$, for which $\sigma \geq 4$. Moreover, we assume that if we formally treat the factor $\nabla Y$ as a factor $\nabla \phi_{u^{\prime}+1}$ in the above equation, then the inductive assumption of Proposition 1.1 can be applied. (See Subsection 3.1 in [A 2010] for a strict discussion of the multiparameter induction by which we prove Proposition 1.1.)

The conclusion (under various assumptions which we will explain below) is: Denote by $\mathrm{H}_{2, \alpha} \subset \mathrm{H}_{2}$ the index set of tensor fields with rank $\alpha$ in (2-2).

We claim that there is a linear combination of acceptable ${ }^{16}$ tensor fields

$$
\sum_{d \in D} a_{d} C_{g}^{d, i_{1} \ldots i_{\alpha+1}}\left(\Omega_{1}, \ldots, \Omega_{p}, Y, \phi_{1}, \ldots, \phi_{u}\right),
$$

each with a simple character $\vec{\kappa}_{\text {simp }}^{\prime}$, so that

$$
\begin{gathered}
\sum_{h \in H_{2, \alpha}} a_{h} C_{g}^{h, i_{1} \ldots i_{\alpha}}\left(\Omega_{1}, \ldots, \Omega_{p}, Y, \phi_{1}, \ldots, \phi_{u^{\prime}}\right) \nabla_{i_{1}} v \ldots \nabla_{i_{\alpha}} v \\
-X_{*} \operatorname{div}_{i_{\alpha+1}} \sum_{d \in D} a_{d} C_{g}^{d, i_{1} \ldots i_{\alpha+1}}\left(\Omega_{1}, \ldots, \Omega_{p}, Y, \phi_{1}, \ldots, \phi_{u^{\prime}}\right) \nabla_{i_{1}} v \ldots \nabla_{i_{\alpha}} v \\
=\sum_{t \in T} a_{t} C_{g}^{t}\left(\Omega_{1}, \ldots, \Omega_{p}, Y, \phi_{1}, \ldots, \phi_{u^{\prime}}, v^{\alpha}\right) .
\end{gathered}
$$

The linear combination on the right-hand side stands for a generic linear combination of complete contractions in the form (2-1) with a factor $\nabla Y$ and with a simple character that is subsequent to $\vec{\kappa}_{\text {simp. }}^{\prime}$.

The assumption under which (2-3) holds is that there should be no tensor fields of rank $\alpha$ in (2-2) that are "bad". Here "bad" means the following:

If $\sigma_{2}=0$ in $\vec{\kappa}_{\text {simp }}^{\prime}$ then a tensor field in the form (2-1) is "bad" provided:

(1) The factor $\nabla Y$ contains a free index.

(2) If we formally erase the factor $\nabla Y$ (which contains a free index), then the resulting tensor field should have no removable indices, ${ }^{17}$ and no free indices. ${ }^{18}$ Moreover, any factors $S_{*} R_{i j k l}$ should be simple.

If $\sigma_{2}>0$ in $\vec{\kappa}_{\text {simp }}^{\prime}$ then a tensor field in the form (2-1) is "bad" provided:

(1) The factor $\nabla Y$ contains a free index.

(2) If we formally erase the factor $\nabla Y$ (which contains a free index), then the resulting tensor field should have no removable indices, any factors $S_{*} R_{i j k l}$ should be simple, any factor $\nabla_{a b}^{(2)} \Omega_{h}$ should have at most one of the indices ${ }_{a},{ }_{b}$ free or contracting against a factor $\nabla \phi_{s}$.

16“Acceptable" in the sense that each factor $\Omega_{i}$ is differentiated at least twice).

${ }^{17}$ Thus, the tensor field should consist of factors $S_{*} R_{i j k l}, \nabla^{(2)} \Omega_{h}$, and factors $\nabla_{r_{1} \ldots r_{m}}^{(m)} R_{i j k l}$ with all the indices $r_{1}, \ldots, r_{m}$ contracting against factors $\nabla \phi_{h}$.

${ }^{18}$ That is $\alpha=1$ in (2-2). 
(3) Any factor $\nabla^{(m)} R_{i j k l}$ can contain at most one (necessarily special, by virtue of (2)) free index.

Furthermore, we claim that the proof of this lemma will only rely on the inductive assumption of Proposition 1.1. Moreover, we claim that if none of the tensor fields indexed in $\mathrm{H}_{2}$ (in (2-2)) have a free index in $\nabla Y$, then we may assume that none of the tensor fields indexed in $D$ in (2-3) have that property either.

Lemma 2.2. We assume that (2-2) holds for $\sigma=3$. We also assume that for each of the tensor fields in $\mathrm{H}_{2}^{\alpha, * 19}$ there is at least one removable index. We then have two claims:

First, the conclusion of Lemma 2.1 holds in this setting. Second, we can write

$$
\begin{gathered}
\sum_{h \in H_{2}} a_{h} X \operatorname{div}_{i_{1}} \ldots X \operatorname{div}_{i_{\alpha}} C_{g}^{h, i_{1} \ldots i_{\alpha}}\left(\Omega_{1}, \ldots, \Omega_{p}, Y, \phi_{1}, \ldots, \phi_{u^{\prime}}\right) \\
=\sum_{q \in Q} a_{q} X \operatorname{div}_{i_{1}} \ldots X \operatorname{div}_{i_{a^{\prime}}} C_{g}^{q, i_{1} \ldots i_{a^{\prime}}}\left(\Omega_{1}, \ldots, \Omega_{p}, Y, \phi_{1}, \ldots, \phi_{u^{\prime}}\right) \\
\quad+\sum_{t \in T} a_{t} C_{g}^{t}\left(\Omega_{1}, \ldots, \Omega_{p}, Y, \phi_{1}, \ldots, \phi_{u^{\prime}}\right)
\end{gathered}
$$

where the linear combination $\sum_{q \in Q} a_{q} C_{g}^{q, i_{1} \ldots i_{a^{\prime}}}$ stands for a generic linear combination of tensor fields in the form

$$
\begin{aligned}
\operatorname{pcontr}( & \nabla^{\left(m_{1}\right)} R_{i j k l} \otimes \cdots \otimes \nabla^{\left(m_{\sigma_{1}}\right)} R_{i j k l} \\
& \otimes S_{*} \nabla^{\left(v_{1}\right)} R_{i j k l} \otimes \cdots \otimes S_{*} \nabla^{\left(v_{t}\right)} R_{i j k l} \otimes \nabla^{(B)} Y \\
& \otimes \nabla^{\left(b_{1}\right)} \Omega_{1} \otimes \cdots \otimes \nabla^{\left(b_{p}\right)} \Omega_{p} \otimes \nabla \phi_{z_{1}} \cdots \otimes \nabla \phi_{z_{w}} \\
& \left.\otimes \nabla \phi_{z_{w+1}}^{\prime} \otimes \cdots \otimes \nabla \phi_{z_{w+d}}^{\prime} \otimes \cdots \otimes \nabla \tilde{\phi}_{z_{w+d+1}} \otimes \cdots \otimes \nabla \tilde{\phi}_{z_{w+d+y}}\right),
\end{aligned}
$$

with $B \geq 2$, with a simple character $\vec{\kappa}_{\text {simp }}^{\prime}$ and with each $a^{\prime} \geq \alpha$. The acceptable complete contractions $C_{g}^{t}\left(\Omega_{1}, \ldots, \Omega_{p}, Y, \phi_{1}, \ldots, \phi_{u^{\prime}}\right)$ are simply subsequent to $\vec{\kappa}_{\text {simp. }}^{\prime} X \operatorname{div}_{i}$ here means that $\nabla_{i}$ is not allowed to hit the factors $\nabla \phi_{h}$ (but it is allowed to hit $\left.\nabla^{(B)} Y\right)$.

For our next two lemmas, we will be considering tensor fields in the general form

$$
\begin{aligned}
\operatorname{contr}\left(\nabla^{\left(m_{1}\right)}\right. & R_{i j k l} \otimes \cdots \otimes \nabla^{\left(m_{s}\right)} R_{i j k l} \\
& \otimes S_{*} \nabla^{\left(v_{1}\right)} R_{i j k l} \otimes \cdots \otimes S_{*} \nabla^{\left(v_{b}\right)} R_{i j k l} \\
& \otimes \nabla_{r_{1} \ldots r_{B}}^{(B,+)}\left(\nabla_{a} \omega_{1} \nabla_{b} \omega_{2}-\nabla_{b} \omega_{1} \nabla_{a} \omega_{1}\right) \\
& \left.\otimes \nabla^{\left(d_{1}\right)} \Omega_{p} \otimes \cdots \otimes \nabla^{\left(d_{p}\right)} \Omega_{p} \otimes \nabla \phi_{1} \otimes \cdots \otimes \nabla \phi_{u}\right) ;
\end{aligned}
$$

${ }^{19}$ Recall from [A 2010] that $H_{2}^{\alpha, *}$ is the index set of tensor fields of rank $\alpha$ in (2-2) with a free index in the factor $\nabla Y$. 
here $\nabla_{r_{1} \ldots r_{B}}^{(B,+)}(\ldots)$ stands for the sublinear combination in $\nabla_{r_{1} \ldots r_{B}}^{(B)}(\ldots)$ where each derivative $\nabla_{r_{i}}$ is not allowed to hit the factor $\nabla \omega_{2}$.

We also recall from [A 2010] that $X_{+} \operatorname{div}_{i}$ stands for the sublinear combination in $X \operatorname{div}_{i}$ where $\nabla_{i}$ is in addition not allowed to hit the factor $\nabla \omega_{2}$ (it is allowed to hit the factor $\left.\nabla^{(B)} \omega_{1}\right)$.

Lemma 2.3. Consider a linear combination of partial contractions,

$$
\sum_{x \in X} a_{x} C_{g}^{x, i_{1} \ldots i_{a}}\left(\Omega_{1}, \ldots, \Omega_{p},\left[\omega_{1}, \omega_{2}\right], \phi_{1}, \ldots, \phi_{u^{\prime}}\right),
$$

where each of the tensor fields $C_{g}^{x, i_{1} \ldots i_{a}}$ is in the form (2-6) with $B=0$ (and is antisymmetric in the factors $\nabla_{a} \omega_{1}, \nabla_{b} \omega_{2}$ by definition), with rank $a \geq \alpha$ and real length $\sigma \geq 4{ }^{20}$ We assume that all these tensor fields have a given simple character which we denote by $\vec{\kappa}_{\text {simp }}^{\prime}$ (we use $u^{\prime}$ instead of $u$ to stress that this lemma holds in generality). We assume that

$$
\begin{aligned}
\sum_{x \in X} a_{x} X_{*} \operatorname{div}_{i_{1}} \ldots X_{*} \operatorname{div}_{i_{a}} C_{g}^{x, i_{1} \ldots i_{a}}\left(\Omega_{1}, \ldots, \Omega_{p},\left[\omega_{1}, \omega_{2}\right], \phi_{1}, \ldots, \phi_{u}\right) \\
+\sum_{j \in J} a_{j} C_{g}^{j}\left(\Omega_{1}, \ldots, \Omega_{p},\left[\omega_{1}, \omega_{2}\right], \phi_{1}, \ldots, \phi_{u}\right)=0,
\end{aligned}
$$

where $X_{*} \operatorname{div}_{i}$ stands for the sublinear combination in $X \operatorname{div}_{i}$ where $\nabla_{i}$ is in addition not allowed to hit the factors $\nabla \omega_{1}, \nabla \omega_{2}$. The contractions $C^{j}$ here are simply subsequent to $\vec{\kappa}_{\text {simp. }}^{\prime}$. We assume that if we formally treat the factors $\nabla \omega_{1}, \nabla \omega_{2}$ as factors $\nabla \phi_{u+1}, \nabla \phi_{u+2}$ (disregarding whether they are contracting against special indices) in the above, then the inductive assumption of Proposition 1.1 applies.

The conclusion we will draw (under various hypotheses that we will explain below) is that we can write

$$
\begin{aligned}
& \sum_{x \in X} a_{x} X_{+} \operatorname{div}_{i_{1}} \ldots X_{+} \operatorname{div}_{i_{a}} C_{g}^{x, i_{1} \ldots i_{a}}\left(\Omega_{1}, \ldots, \Omega_{p},\left[\omega_{1}, \omega_{2}\right], \phi_{1}, \ldots, \phi_{u}\right) \\
& =\sum_{x \in X^{\prime}} a_{x} X_{+} \operatorname{div}_{i_{1}} \ldots X_{+} \operatorname{div}_{i_{a}} C_{g}^{x, i_{1} \ldots i_{a}}\left(\Omega_{1}, \ldots, \Omega_{p},\left[\omega_{1}, \omega_{2}\right], \phi_{1}, \ldots, \phi_{u}\right) \\
& \quad+\sum_{j \in J} a_{j} C_{g}^{j}\left(\Omega_{1}, \ldots, \Omega_{p},\left[\omega_{1}, \omega_{2}\right], \phi_{1}, \ldots, \phi_{u}\right)=0
\end{aligned}
$$

where the tensor fields indexed in $X^{\prime}$ on the right-hand side are in the form (2-6) with $B>0$. All the other sublinear combinations are as above.

Assumptions needed for (2-8): We claim that (2-8) holds under certain assumptions on the $\alpha$-tensor fields in (2-7) that have rank $\alpha$ and have a free index in one

${ }^{20}$ Recall that in the definition of "real length" in this setting, we count each factor $\nabla^{(m)} R$, $S_{*} \nabla^{(v)} R, \nabla^{(B)} \Omega_{x}$ once, the two factors $\nabla^{(a)} \omega_{1}, \nabla \omega_{2}$ as one, and the factors $\nabla \phi, \nabla \phi^{\prime}, \nabla \tilde{\phi}$ as nothing. 
of the factors $\nabla \omega_{1}, \nabla \omega_{2}$ (say in $\nabla \omega_{1}$ without loss of generality) - we denote the index set of those tensor fields by $X^{\alpha, *} \subset X$.

The assumption we need in order for the claim to hold is that no tensor field indexed in $X^{\alpha, *}$ should be "bad". A tensor field is "bad" if it has the property that when we erase the expression $\nabla_{[a} \omega_{1} \nabla_{b]} \omega_{2}$ (and make the index that contracted against ${ }_{b}$ into a free index) then the resulting tensor field will have no removable indices, and all factors $S_{*} R_{i j k l}$ will be simple.

Lemma 2.4. We assume that (2-7) holds, where now the tensor fields have length $\sigma=3$. We also assume that for each of the tensor fields indexed in $X$, there is a removable index in each of the real factors. We then claim that the conclusion of Lemma 2.3 is still true in this setting.

For the most part, the remainder of this paper is devoted to proving the above lemmas. However, we first state and prove some further technical claims, one of which appeared as Lemma A.1 in [A 2010]. ${ }^{21}$

2B. Two more technical lemmas. We claim that an analogue of Lemma 4.10 in [A 2010] $]^{22}$ can be derived for tensor fields with a given simple character $\vec{\kappa}_{\text {simp }}$, and where rather than having one additional factor $\nabla \phi_{u+1}$ (which is not encoded in the simple character $\vec{\kappa}_{\text {simp }}$ ), we have two additional factors $\nabla_{a} \phi_{u+1}, \nabla_{b} \phi_{u+2}$.

Lemma 2.5. Consider a linear combination

$$
\sum_{l \in L} a_{l} C_{g}^{l, i_{1} \ldots i_{\beta}}\left(\Omega_{1}, \ldots, \Omega_{p}, \phi_{1}, \ldots, \phi_{u}\right)
$$

of acceptable tensor fields in the form (1-6) with a given $u$-simple character $\vec{\kappa}_{\text {simp. }}$. Assume that the minimum rank among those tensor fields above is $\alpha \geq 2$. Assume that

$$
\begin{array}{r}
\sum_{l \in L} a_{l} X_{*} \operatorname{div}_{i_{3}} \ldots X_{*} \operatorname{div}_{i_{\beta}} C_{g}^{l, i_{1} \ldots i_{\beta}}\left(\Omega_{1}, \ldots, \Omega_{p}, \phi_{1}, \ldots, \phi_{u}\right) \\
\times \nabla_{i_{1}} \phi_{u+1} \nabla_{i_{2}} \phi_{u+2} \\
+\sum_{j \in J} a_{j} C_{g}^{j, i_{1} i_{2}}\left(\Omega_{1}, \ldots, \Omega_{p}, \phi_{1}, \ldots, \phi_{u}\right) \nabla_{i_{1}} \phi_{u+1} \nabla_{i_{2}} \phi_{u+2}=0
\end{array}
$$

here $X_{*} \operatorname{div}_{i}$ means that $\nabla^{i}$ is in addition not allowed to hit either of the factors $\nabla \phi_{u+1}, \nabla \phi_{u+2}$. We also assume that if we formally treat the factors $\nabla \phi_{u+1}$, $\nabla \phi_{u+2}$ as factors $\nabla \phi_{u+1}, \nabla \phi_{u+2}$ then (2-9) falls under the inductive assumption of Proposition 1.1 (with respect to the parameters $(n, \sigma, \Phi, u)$ ). Denote by $L^{\alpha} \subset L$

${ }^{21}$ Its proof was also deferred to the present paper.

${ }^{22}$ This corresponds to Lemma 4.44 in [A 2012]. 
the index set of terms with rank $\alpha$. We additionally assume that none of the tensor fields $C_{g}^{l, i_{1} \ldots i_{\beta}}\left(\Omega_{1}, \ldots, \Omega_{p}, \phi_{1}, \ldots, \phi_{u}\right)$ are "forbidden," in the sense defined above Proposition 2.1 in [A 2010].

We then claim that there exists a linear combination of $(\alpha+1)$-tensor fields with a u-simple character $\vec{\kappa}_{\text {simp }}$ (indexed in $Y$ below) so that

$$
\begin{aligned}
& \sum_{l \in L^{\alpha}} a_{l} C_{g}^{l, i_{1} \ldots i_{\alpha}}\left(\Omega_{1}, \ldots, \Omega_{p}, \phi_{1}, \ldots, \phi_{u}\right) \nabla_{i_{1}} \phi_{u+1} \nabla_{i_{2}} \phi_{u+2} \nabla_{i_{3}} v \ldots \nabla_{i_{\alpha}} v \\
&=X_{*} \operatorname{div}_{i_{\alpha+1}} \sum_{y \in Y} a_{y} C_{g}^{l, i_{1} \ldots i_{\alpha+1}}\left(\Omega_{1}, \ldots, \Omega_{p}, \phi_{1}, \ldots, \phi_{u}\right) \\
& \times \nabla_{i_{1}} \phi_{u+1} \nabla_{i_{2}} \phi_{u+2} \nabla_{i_{3}} v \ldots \nabla_{i_{\alpha}} v \\
&+\sum_{j \in J} a_{j} C_{g}^{j, i_{1} \ldots i_{\alpha}}\left(\Omega_{1}, \ldots, \Omega_{p}, \phi_{1}, \ldots, \phi_{u}\right) \\
& \times \nabla_{i_{1}} \phi_{u+1} \nabla_{i_{2}} \phi_{u+2} \nabla_{i_{3}} v \ldots \nabla_{i_{\alpha}} v .
\end{aligned}
$$

We also claim that we can write

$$
\begin{aligned}
& \sum_{l \in L} a_{l} X \operatorname{div}_{i_{3}} \ldots X \operatorname{div}_{i_{\beta}} C_{g}^{l, i_{1} \ldots i_{\beta}}\left(\Omega_{1}, \ldots, \Omega_{p}, \phi_{1}, \ldots, \phi_{u}\right) \nabla_{i_{1}} \phi_{u+1} \nabla_{i_{2}} \phi_{u+2} \\
& =\sum_{j \in J} a_{j} C_{g}^{j}\left(\Omega_{1}, \ldots, \Omega_{p}, \phi_{1}, \ldots, \phi_{u}\right) \\
& +\sum_{q \in Q_{1}} a_{q} X \operatorname{div}_{i_{3}} \ldots X \operatorname{div}_{i_{\alpha}} \\
& \quad \times C_{g}^{q, i_{3} \ldots i_{\alpha}}\left(\Omega_{1}, \ldots, \Omega_{p}, \phi_{1}, \ldots, \phi_{u+2}\right) \nabla_{i_{1}} \phi_{u+1} \nabla_{i_{2}} \phi_{u+2} \\
& +\sum_{q \in Q_{2}} a_{q} X \operatorname{div}_{i_{3}} \ldots X \operatorname{div}_{i_{\alpha}} \\
& \quad \times C_{g}^{q, i_{3} \ldots i_{\alpha}}\left(\Omega_{1}, \ldots, \Omega_{p}, \phi_{1}, \ldots, \phi_{u+2}\right),
\end{aligned}
$$

where the tensor fields indexed in $Q_{1}$ are acceptable with a u-simple character $\vec{\kappa}_{\text {simp }}$ and with a factor $\nabla^{(2)} \phi_{u+1}$ and a factor $\nabla \phi_{u+2}$. The tensor fields indexed in $Q_{2}$ are acceptable with a $u$-simple character $\vec{\kappa}_{\text {simp }}$ and with a factor $\nabla^{(2)} \phi_{u+2}$ and a factor $\nabla \phi_{u+1}$.

Proof of Lemma 2.5. We may divide the index set $L^{\alpha}$ into subsets $L_{I}^{\alpha}, L_{I I}^{\alpha}$ according to whether the two factors $\nabla \phi_{u+1}, \nabla \phi_{u+2}$ are contracting against the same factor or not - we will then prove our claim for those two index sets separately. Our claim for the index set $L_{I I}^{\alpha}$ follows by a straightforward adaptation of the proof of Lemma 4.10 in [A 2010]. (Notice that the forbidden cases of the present lemma are exactly in correspondence with the forbidden cases of that lemma.) Therefore, we now prove our claim for the index set $L_{I}^{\alpha}$ :

We denote by $L_{I} \subset L, J_{I} \subset J$ the index sets of terms for which the two factors $\nabla \phi_{u+1}, \nabla \phi_{u+2}$ are contracting against the same factor. It then follows that (2-9) holds with the index sets $L, J$ replaced by $L_{I}, J_{I}$ - denote the resulting new equation by New(2-9). Now, for each tensor field $C_{g}^{l, i_{1} \ldots i_{\beta}}$ and each complete contraction 
$C_{g}^{j}$, we let $\operatorname{Sym}\left[C_{g}^{l, i_{1} \ldots i_{\beta}}\right], \operatorname{Sym}\left[C_{g}^{l, i_{1} \ldots i_{\beta}}\right], \operatorname{AntSym}\left[C_{g}^{j}\right], \operatorname{AntSym}\left[C_{g}^{j}\right]$ stand for the tensor field/complete contraction that arises from $C_{g}^{l, i_{1} \ldots i_{\beta}}, C_{g}^{j}$ by symmetrizing (respectively antisymmetrizing) the indices ${ }_{a},{ }_{b}$ in the two factors $\nabla_{a} \phi_{u+1}, \nabla_{b} \phi_{u+2}$. We accordingly derive two new equations from New(2-9), which we denote by New(2-9) Sym and New(2-9) AntSym.

We will then prove the claim separately for the tensor fields in the sublinear combination $\sum_{l \in L_{I}^{\alpha}} a_{l} \operatorname{Sym}[C]_{g}^{l, i_{1} \ldots i_{\alpha}}$ and the tensor fields in the sublinear combination $\sum_{l \in L_{I}^{\alpha}} a_{l} \operatorname{AntSym}[C]_{g}^{l, i_{1} \ldots i_{\alpha}}$.

The claim (2-10) for the sublinear combination $\sum_{l \in L_{I}^{\alpha}} a_{l}$ AntSym $[C]_{g}^{l, i_{1} \ldots i_{\alpha}}$ follows directly from the arguments in the proof of Lemma 2.3 (see this proof below). Therefore it suffices to show our claim for $\sum_{l \in L_{I}^{\alpha}} a_{l} \operatorname{Sym}[C]_{g}^{l, i_{1} \ldots i_{\alpha}}$.

We prove this claim as follows: We divide the index set $L_{I}^{\alpha}$ according to the form of the factor against which the two factors $\nabla \phi_{u+1}, \nabla \phi_{u+2}$ contract: List out the nongeneric factors $\left\{T_{1}, \ldots, T_{a}\right\}$ in $\vec{\kappa}_{\text {simp. }}{ }^{23}$ Then, for each $k \leq a$ we let $L_{I, k}^{\alpha}$ stand for the index set of terms for which the factors $\nabla \phi_{u+1}, \nabla \phi_{u+2}$ are contracting against the factor $T_{k}$. We also let $L_{I, a+1}^{\alpha}$ stand for the index set of terms for which the factors $\nabla \phi_{u+1}, \nabla \phi_{u+2}$ are contracting against a generic factor $\nabla^{(m)} R_{i j k l}$. We will prove our claim for each of the sublinear combinations $\sum_{l \in L_{I, a+1}^{\alpha}} a_{l} \operatorname{Sym}[C]_{g}^{l, i_{1} \ldots i_{\alpha}}$ separately.

We first observe that for each $k \leq a+1$, we may obtain a new true equation from (2-9) by replacing $L$ by $L_{I, a+1}$ - denote the resulting equation by (2-9) $I$, Sym, $k$. Therefore, for each $k \leq a+1$ for which $T_{k}$ is in the form $\nabla^{(p)} \Omega_{h}$, our claim follows straightforwardly by applying Corollary 1 from [A 2010]. ${ }^{24}$

Now, we consider the case where the factor $T_{k}$ is in the form $S_{*} \nabla^{(v)} R_{a b c d}$ : In that case we denote by $L_{I, k, \sharp}$ the index set of terms for which one of the factors $\nabla \phi_{u+1}, \nabla \phi_{u+2}$ is contracting against a special index in $T_{k}$. In particular, we will let $L_{I, k, \sharp}^{\alpha} \subset L_{I, k, \sharp}$ stand for the index set of terms with rank $\alpha$. We will then show that two equations hold:

${ }^{23}$ Recall from [A 2010] that the nongeneric factors in $\vec{\kappa}_{\text {simp }}$ are all the factors in the form $\nabla^{(A)} \Omega_{h}, S_{*} \nabla^{(v)} R_{i j k l}$, and also all the factors $\nabla^{(m)} R_{i j k l}$ that contract against at least one factor $\nabla \phi_{s}$.

${ }^{24}$ This corresponds to Corollary 4.14 in [A 2012]. There is no danger of falling under a "forbidden case," since we started with tensor fields which were not forbidden. 
First, we claim that there exists a linear combination of tensor fields as claimed in (2-10) so that

(2-12)

$$
\begin{aligned}
&\left.\sum_{l \in L_{I, k, \sharp}^{\alpha} a_{l} \operatorname{Sym}[C]_{g}^{l, i_{1} \ldots i_{\alpha}}\left(\Omega_{1}, \ldots, \Omega_{p}, \phi_{1},\right.}, \ldots, \phi_{u}\right) \\
& \times \nabla_{i_{1}} \phi_{u+1} \nabla_{i_{2}} \omega \nabla_{i_{3}} v \ldots \nabla_{i_{\alpha}} v \\
&-\sum_{y \in Y} a_{y} X \operatorname{div}_{i_{\alpha+1}} C_{g}^{y, i_{1} \ldots i_{\alpha+1}}\left(\Omega_{1}, \ldots, \Omega_{p}, \phi_{1}, \ldots, \phi_{u}\right) \\
& \times \nabla_{i_{1}} \phi_{u+1} \nabla_{i_{2}} \omega \nabla_{i_{3}} v \ldots \nabla_{i_{\alpha}} v
\end{aligned}
$$




$$
\begin{array}{r}
=\sum_{l \in L_{O K}^{\alpha}} a_{l} X \operatorname{div}_{i_{\alpha+1}} C_{g}^{l, i_{1} \ldots i_{\alpha} i_{\alpha+1}} \nabla_{i_{1}} \phi_{u+1} \nabla_{i_{2}} \omega \nabla_{i_{3}} v \ldots \nabla_{i_{\alpha}} v \\
+\sum_{j \in J} a_{j} C_{g}^{j, i_{1} \ldots i_{\alpha}}\left(\Omega_{1}, \ldots, \Omega_{p}, \phi_{1}, \ldots, \phi_{u}\right) \\
\times \nabla_{i_{1}} \phi_{u+1} \nabla_{i_{2}} \omega \nabla_{i_{3}} v \ldots \nabla_{i_{\alpha}} v
\end{array}
$$

where the tensor fields in $L_{O K}^{\alpha}$ have all the properties of the terms in $L_{I, k}$, rank $\alpha$ and furthermore none of the factors $\nabla \phi_{u+1}, \nabla \phi_{u+2}$ are contracting against a special index.

Then (under the assumption that $L_{I, k, \sharp}^{\alpha}=\varnothing$ ) we claim that we can write

$$
\begin{aligned}
& \sum_{l \in L_{I, k, \sharp}} a_{l} X \operatorname{div}_{i_{3}} \ldots X \operatorname{div}_{i_{\beta}} \\
& \times \operatorname{Sym}[C]_{g}^{l, i_{1} \ldots i_{\beta}}\left(\Omega_{1}, \ldots, \Omega_{p}, \phi_{1}, \ldots, \phi_{u}\right) \nabla_{i_{1}} \phi_{u+1} \nabla_{i_{2}} \phi_{u+2} \\
& =\sum_{l \in L_{I, k, O K}} a_{l} X \operatorname{div}_{i_{3}} \ldots X \operatorname{div}_{i_{\beta}} \\
& \quad \times \operatorname{Sym}[C]_{g}^{l, i_{1} \ldots i_{\beta}}\left(\Omega_{1}, \ldots, \Omega_{p}, \phi_{1}, \ldots, \phi_{u}\right) \nabla_{i_{1}} \phi_{u+1} \nabla_{i_{2}} \phi_{u+2} \\
& \quad+\sum_{j \in J} a_{j} \operatorname{Sym}[C]_{g}^{j, i_{1} i_{2}}\left(\Omega_{1}, \ldots, \Omega_{p}, \phi_{1}, \ldots, \phi_{u}\right) \nabla_{i_{1}} \phi_{u+1} \nabla_{i_{2}} \phi_{u+2},
\end{aligned}
$$

where the tensor fields in $L_{I, k, O K}$ have all the properties of the terms in $L_{I, k}$, but they additionally have rank $\geq \alpha+1$ and furthermore none of the factors $\nabla \phi_{u+1}, \nabla \phi_{u+2}$ are contracting against a special index.

If we can show the above two equations, then we are reduced to showing our claim under the additional assumption that no tensor field indexed in $L$ in $\operatorname{Sym}(2-9)$ has any factors $\nabla \phi_{u+1}, \nabla \phi_{u+2}$ that contract against a special index in $T_{k}$. Under that assumption, we may additionally assume that none of the complete contractions indexed in $J$ in (2-9) have that property. ${ }^{25}$ Therefore, we may then erase the factor $\nabla \phi_{u+1}$ from all the complete contractions and tensor fields in (2-9) $k$ by virtue of the operation Erase, introduced in the Appendix in [A 2012] - our claim then follows by applying Corollary 1 from [A 2010] to the resulting equation and then reintroducing the erased factor $\nabla \phi_{u+1}$.

Outline of the proofs of (2-12) and (2-13). First we prove (2-12): Suppose without loss of generality that $T_{k}$ contracts against $\nabla \tilde{\phi}_{1}$ and $\nabla \phi_{2}^{\prime}, \ldots, \nabla \phi_{h}^{\prime}$; then replace the two factors $\nabla_{a} \phi_{1}, \nabla_{b} \phi_{u+1}$ by $g_{a b}$ and then apply Ricto $\Omega_{p+1}{ }^{26}$ (obtaining a new true equation) an then apply the eraser to the resulting true equation. We then apply Corollary 1 from [A 2010] to the resulting equation, ${ }^{27}$ and finally we replace

${ }^{25}$ This can be derived by repeating the proof of (2-12), (2-13).

${ }^{26}$ See the relevant lemma in the Appendix of [A 2012].

${ }^{27}$ Since the factor $\nabla \phi_{u+2}$ survives this operation, and since we started out with terms that were not "forbidden," there is no danger of falling under a "forbidden case" of Corollary 1 from [A 2010]. 
the factor $\nabla_{r_{1} \ldots r_{b}}^{(b)} \Omega_{p+1}$ by an expression

$$
S_{*} \nabla_{y_{2} \ldots y_{h} r_{1} \ldots r_{b-1}}^{(b+h-1)} R_{i j k r_{b}} \nabla^{i} \tilde{\phi}_{1} \nabla^{j} \phi_{u+2} \nabla^{k} \phi_{u+1} \nabla^{y_{2}} \phi_{2}^{\prime} \ldots \nabla^{y_{h}} \phi_{h}^{\prime} .
$$

As in the proof of Lemma 4.10 in [A 2010], we derive our claim. Then (2-13) is proven by iteratively applying this step and making each $\nabla v$ into an $X$ div at every stage.

We analogously show our claim when the factor $T_{k}$ is in the form $\nabla^{(m)} R_{i j k l}$ : In that case we denote by $L_{I, k, \sharp}$ the index set of terms for which both the factors $\nabla \phi_{u+1}, \nabla \phi_{u+2}$ are contracting against a special index in $T_{k}$. We will then show two claims:

First, that there exists a linear combination of partial contractions (indexed in $Y$ below) as claimed in (2-10) so that

$$
\begin{aligned}
& \sum_{l \in L_{l, k, \sharp}^{\alpha}} a_{l} \operatorname{Sym}[C]_{g}^{l, i_{1} \ldots i_{\alpha}}\left(\Omega_{1}, \ldots, \Omega_{p}, \phi_{1}, \ldots, \phi_{u}\right) \nabla_{i_{1}} \phi_{u+1} \nabla_{i_{2}} \omega \nabla_{i_{3}} v \ldots \nabla_{i_{\alpha}} v \\
& =\sum_{y \in Y} a_{y} X \operatorname{div}_{i_{\alpha+1}} C_{g}^{y, i_{1} \ldots i_{\alpha+1}}\left(\Omega_{1}, \ldots, \Omega_{p}, \phi_{1}, \ldots, \phi_{u}\right) \\
& \times \nabla_{i_{1}} \phi_{u+1} \nabla_{i_{2}} \omega \nabla_{i_{3}} v \ldots \nabla_{i_{\alpha}} v \\
& +\sum_{l \in L_{O K}^{\alpha}} a_{l} X \operatorname{div}_{i_{\alpha+1}} C_{g}^{l, i_{1} \ldots i_{\alpha} i_{\alpha+1}} \nabla_{i_{1}} \phi_{u+1} \nabla_{i_{2}} \omega \nabla_{i_{3}} v \ldots \nabla_{i_{\alpha}} v \\
& \begin{array}{r}
+\sum_{j \in J} a_{j} C_{g}^{j, i_{1} \ldots i_{\alpha}}\left(\Omega_{1}, \ldots, \Omega_{p}, \phi_{1}, \ldots, \phi_{u}\right) \\
\times \nabla_{i_{1}} \phi_{u+1} \nabla_{i_{2}} \omega \nabla_{i_{3}} v \ldots \nabla_{i_{\alpha}} v,
\end{array}
\end{aligned}
$$

where the tensor fields in $L_{O K}^{\alpha}$ have all the properties of the terms in $L_{I, k}$, but they additionally have rank $\alpha$ and furthermore one of the factors $\nabla \phi_{u+1}, \nabla \phi_{u+2}$ does not contract against a special index. Then (under the assumption that $L_{I, k, \sharp}^{\alpha}=\varnothing$ ) we denote by $L_{I, k, \sharp}$ the sublinear combination of terms in $L_{I, k}$ where both factors $\nabla \phi_{u+1}$ or $\nabla \phi_{u+1}$ contract against a special index in $T_{k}$. We claim that we can write

$$
\begin{aligned}
& \sum_{l \in L_{I, k, \sharp}} a_{l} X \operatorname{div}_{i_{3}} \ldots X \operatorname{div}_{i_{\beta}} \\
& \times \operatorname{Sym}[C]_{g}^{l, i_{1} \ldots i_{\beta}}\left(\Omega_{1}, \ldots, \Omega_{p}, \phi_{1}, \ldots, \phi_{u}\right) \nabla_{i_{1}} \phi_{u+1} \nabla_{i_{2}} \phi_{u+2} \\
& =\sum a_{l} X \operatorname{div}_{i_{3}} \ldots X \operatorname{div}_{i_{\beta}} \\
& \quad \times \operatorname{Sym}[C]_{g}^{l, i_{1} \ldots i_{\beta}}\left(\Omega_{1, k, O K}, \ldots, \Omega_{p}, \phi_{1}, \ldots, \phi_{u}\right) \nabla_{i_{1}} \phi_{u+1} \nabla_{i_{2}} \phi_{u+2} \\
& +\sum_{j \in J} a_{j} \operatorname{Sym}[C]_{g}^{j, i_{1} i_{2}}\left(\Omega_{1}, \ldots, \Omega_{p}, \phi_{1}, \ldots, \phi_{u}\right) \nabla_{i_{1}} \phi_{u+1} \nabla_{i_{2}} \phi_{u+2} \text {, }
\end{aligned}
$$

where the tensor fields in $L_{I, k, O K}$ have all the properties of the terms in $L_{I, k}$, but they additionally have rank $\geq \alpha+1$ and furthermore one of the factors $\nabla \phi_{u+1}$, $\nabla \phi_{u+2}$ does not contract against a special index. 
If we can show the above two equations, then we are reduced to showing our claim under the additional assumption that no tensor field indexed in $L$ in $\operatorname{Sym}(2-9)$ has the two factors $\nabla \phi_{u+1}, \nabla \phi_{u+2}$, contracting against a special index in $T_{k}$. Under that assumption, we may additionally assume that none of the complete contractions indexed in $J$ in (2-9) have that property. Therefore, we may then erase the factor $\nabla \phi_{u+1}$ from all the complete contractions and tensor fields in (2-9) $)_{k}$ - our claim then follows by applying Lemma 4.10 in [A 2010] to the resulting equation ${ }^{28}$ and then reintroducing the erased factor $\nabla \phi_{u+1}$.

Outline of the proofs of (2-14) and (2-15). First we prove (2-14). Suppose without loss of generality that $T_{k}$ contracts against $\nabla \phi_{1}, \ldots, \nabla \phi_{h}$ (possibly with $h=0$ ); then replace the two factors $\nabla_{a} \phi_{1}, \nabla_{b} \phi_{u+1}$ by $g_{a b}$ and then apply Ricto $\Omega_{p+1}$ (obtaining a new true equation), and then apply the eraser to the factors $\nabla \phi_{1}, \ldots, \nabla \phi_{h}$ in the resulting true equation. Then (apart from the cases, discussed below, where the above operation may lead to a "forbidden case" of Corollary 1 in [A 2010]), we apply that corollary to the resulting equation, and finally we replace the factor $\nabla_{r_{1} \ldots r_{b}}^{(b)} \Omega_{p+1}$ by an expression

$$
\nabla_{s_{1} \ldots s_{h} r_{1} \ldots r_{b-2}}^{(b+h)} R_{i r_{b-1} k r_{b}} \nabla^{i} \phi_{u+1} \nabla^{k} \phi_{u+2} \nabla^{s_{1}} \phi_{1} \ldots \nabla^{s_{h}} \phi_{h} .
$$

As in the proof of Lemma 4.10 in [A 2010], we derive our claim. Then (2-14) is proven by iteratively applying this step and making each $\nabla v$ into an $X$ div at every stage (again, provided we never encounter "forbidden cases"). If we do encounter forbidden cases, then our claims follow by just making the factors $\nabla \phi_{u+1}, \nabla \phi_{u+2}$ into $X$ divs and then applying Corollary 1 in [A 2012] to the resulting equation (the resulting equation is not forbidden, since it will contain a factor $\nabla^{(m)} R_{i j k l}$ with two free indices), and in the end renaming two factors $\nabla v$ as $\nabla \phi_{u+1}, \nabla \phi_{u+2}$.

\section{A further generalization: Proof of Lemma A.1 from [A 2010]. We remark that} on a few occasions later in this series of papers we will be using a generalized version of the Lemma 2.5. The generalized version asserts that the claim of Lemma 2.5 remains true, for the general case where rather than one or two "additional" factors $\nabla \phi_{u+1}, \nabla \phi_{u+2}$ we have $\beta \geq 3$ "additional" factors $\nabla \phi_{u+1}, \ldots, \nabla \phi_{u+\beta}$. Moreover, in that case there are no "forbidden cases".

\section{Lemma 2.6. Let}

$$
\begin{aligned}
& \sum_{l \in L_{1}} a_{l} C_{g}^{l, i_{1} \ldots i_{\mu}, i_{\mu+1} \ldots i_{\mu+\beta}}\left(\Omega_{1}, \ldots, \Omega_{p}, \phi_{1}, \ldots, \phi_{u}\right) \\
& \sum_{l \in L_{2}} a_{l} C_{g}^{l, i_{1} \ldots i_{b_{l}}, i_{b_{l}+1} \ldots i_{b_{l}+\beta}}\left(\Omega_{1}, \ldots, \Omega_{p}, \phi_{1}, \ldots, \phi_{u}\right),
\end{aligned}
$$

${ }^{28}$ Notice that there is no danger of falling under a "forbidden case" of that lemma, since there will be a nonsimple factor $S_{*} \nabla^{(v)} R_{i j k l}$ by virtue of the factor $\nabla \phi_{u+2}$. 
stand for two linear combinations of acceptable tensor fields in the form (1-6), each with $u$-simple character $\vec{\kappa}_{\text {simp. }}$. We assume that the terms indexed in $L_{1}$ have rank $\mu+\beta$, while the ones indexed in $L_{2}$ have rank greater than $\mu+\beta$.

Assume that

$$
\begin{aligned}
& \sum_{l \in L_{1}} a_{l} X \operatorname{div}_{i_{\beta+1}} \ldots X \operatorname{div}_{i_{\mu+\beta}} \\
& \quad \times C_{g}^{l, i_{1} \ldots i_{\mu+\beta}}\left(\Omega_{1}, \ldots, \Omega_{p}, \phi_{1}, \ldots, \phi_{u}\right) \nabla_{i_{1}} \phi_{u+1} \ldots \nabla_{i_{\beta}} \phi_{u+\beta} \\
& +\sum_{l \in L_{2}} a_{l} X \operatorname{div}_{i_{\beta+1}} \ldots X \operatorname{div}_{i_{b_{l}}} \\
& \quad \times C_{g}^{l, i_{1} \ldots i_{b_{l}+\beta}}\left(\Omega_{1}, \ldots, \Omega_{p}, \phi_{1}, \ldots, \phi_{u}\right) \nabla_{i_{1}} \phi_{u+1} \ldots \nabla_{i_{\beta}} \phi_{u+\beta}
\end{aligned}
$$

modulo terms of length $\geq \sigma+u+\beta+1$. Furthermore, we assume that the above equation falls under the inductive assumption of Proposition 2.1 in [A 2010] (with regard to the parameter weights, $\sigma, \Phi, p$ ). We are not excluding any "forbidden cases".

We claim that there exists a linear combination of $(\mu+\beta+1)$-tensor fields in the form (1-6) with $u$-simple character $\vec{\kappa}_{\text {simp }}$ and length $\sigma+u$ (indexed in $H$ below) such that

$$
\begin{aligned}
& \sum_{l \in L_{1}} a_{l} C_{g}^{l, i_{1} \ldots i_{\mu+\beta}}\left(\Omega_{1}, \ldots, \Omega_{p}, \phi_{1}, \ldots, \phi_{u}\right) \\
& \quad \times \nabla_{i_{1}} \phi_{u+1} \ldots \nabla_{i_{\beta}} \phi_{u+\beta} \nabla_{i_{\beta+1}} v \ldots \nabla_{i_{\beta+\mu}} v
\end{aligned}
$$

modulo terms of length $\geq \sigma+u+\beta+\mu+1$. The terms indexed in $J$ here are $u$-simply subsequent to $\vec{\kappa}_{\text {simp. }}$.

Proof of Lemma 2.6. The proof of the above is a straightforward adaptation of the proof of Lemma 2.5, except for the cases where the tensor fields $C_{g}^{l, i_{1} \ldots i_{\mu}, i_{\mu+1} \ldots i_{\mu+\beta}}$ are "bad," where "bad" in this case means that all factors are in the form $R_{i j k l}$, $S_{*} R_{i j k l}, \nabla^{(2)} \Omega_{h},{ }^{29}$ and in addition each factor $\nabla^{(2)} \Omega_{h}$ contracts against at most one factor $\nabla \phi_{h}, 1 \leq h \leq u+\beta$. So we now focus on that case.

${ }^{29}$ Notice that if this property holds for one of the terms $C_{g}^{l, i_{1} \ldots i_{\mu}, i_{\mu+1} \ldots i_{\mu+\beta}}$, then it will hold for all of them by weight considerations. 
The "bad" case. Let us observe that by weight considerations, all tensor fields in (2-9) must now have rank $\mu$.

We recall that this special proof applies only in the case where there are special free indices in factors $S_{*} R_{i j k l}$ among the tensor fields of minimum rank in (2-9). (If there were no such terms, then the regular proof of Lemma 2.5 would apply.) We distinguish three cases: Either $p>0$, or $p=0$ and $\sigma_{1}>0$, or $p=\sigma_{1}=0$ and $\sigma_{2}>0$. We will prove the above by an induction on the parameters (weight), $\sigma$ : Suppose that the weight of the terms in (2-16) is $-K$ and the real length is $\sigma \geq 3$. We assume that the lemma holds when the Equation (2-16) consists of terms with weight $-K^{\prime}, K^{\prime}<K$, or of terms with weight $-K$ and real length $\sigma^{\prime}, 3 \leq \sigma^{\prime}<\sigma$. The case $p>0$. We first consider the $\mu$-tensor fields in (2-9) with the extra factor $\nabla \phi_{u+1}$ contracting against a factor $\nabla^{(2)} \Omega_{h}$. Denote the index set of those terms by $\bar{L}_{\mu}$. We will first prove that

$$
\begin{aligned}
\sum_{l \in \bar{L}_{\mu}} a_{l} C_{g}^{l, i_{1} \ldots i_{\mu+\beta}}\left(\Omega_{1}, \ldots, \Omega_{p},\right. & \left.\phi_{1}, \ldots, \phi_{u}\right) \\
& \times \nabla_{i_{1}} \phi_{u+1} \ldots \nabla_{i_{\beta}} \phi_{u+\beta} \nabla_{i_{\beta+1}} v \ldots \nabla_{i_{\beta+\mu}} v=0 .
\end{aligned}
$$

It suffices to prove the above for the sublinear combination of $\mu$-tensor fields where $\nabla \phi_{u+1}$ contracts against $\nabla^{(2)} \Omega_{1}$. (2-18) will then follow by relabeling the functions $\Omega_{1}, \ldots, \Omega_{p}$ and repeating this step $p$ times.

We start by a preparatory claim: Let us denote by $\bar{L}_{\mu, \sharp} \subset \bar{L}_{\mu}$ the index set of $\mu$-tensor fields for which the factor $\nabla^{(2)} \Omega_{1}$ contains a free index, say the index $i_{1}$ without loss of generality. We will first prove that

$$
\sum_{l \in \bar{L}_{\mu, \sharp}} a_{l} C_{g}^{l, i_{1} \ldots i_{\mu}} \nabla_{i_{1}} \phi_{u+1} \ldots \nabla_{i_{\beta}} \phi_{u+\beta} \nabla_{i_{\beta+1}} v \ldots \nabla_{i_{\beta+\mu}} v=0 .
$$

Proof of (2-19). We will use the technique (introduced in Subsection 3.1 of [A 2011]) of "inverse integration by parts" followed by the silly divergence formula.

Let us denote by $\hat{C}_{g}^{l}$ the complete contraction that arises from each $C_{g}^{l, i_{1} \ldots i_{\mu+\beta}}$ by formally erasing the expression $\nabla_{s i_{1}}^{(2)} \Omega_{1} \nabla^{s} \phi_{u+1}$ and then making all free indices $i_{\beta+1}, \ldots, i_{\beta+\mu}$ into internal contractions. ${ }^{30}$ Then, the "inverse integration by parts" implies a new integral equation

$$
\int_{M^{n}} \sum_{l \in L_{\mu}} a_{l} \hat{C}_{g}^{l}+\sum_{j \in J} a_{j} C_{g}^{j}+\sum_{z \in Z} a_{z} C_{g}^{z} d V_{g}=0 .
$$

Here the complete contractions indexed in $J$ have length $\sigma+u$ and $u$ factors $\nabla \phi_{u}$, but they are simply subsequent to the simple character $\vec{\kappa}_{\text {simp }}$. The terms indexed in

\footnotetext{
${ }^{30}$ We recall that to "make a free index $i_{y}$ into an internal contraction" means that we add a derivative $\nabla_{i_{y}}$ onto the factor $T_{i_{y}}$ to which the free index $i_{y}$ belongs. The new derivative index $\nabla^{i_{y}}$ is then contracted against the index $i_{y}$ in $T_{i_{y}}$.
} 
$Z$ either have length $\geq \sigma+u+1$ or have length $\sigma+u$, but also have at least one factor $\nabla^{(B)} \phi_{h}$ with $B \geq 2$.

Now, in the above, we consider the complete contractions indexed in $\bar{L}_{\mu, \sharp} \subset \bar{L}_{\mu}$ and we "pull out" the expression $\Delta \nabla_{t} \Omega_{1} \nabla^{t} \phi_{u+1}$ to write

$$
\sum_{l \in L_{\mu, \sharp}} a_{l} \hat{C}_{g}^{l}=\sum_{l \in L_{\mu, \sharp}} a_{l} \bar{C}_{g}^{l} \cdot\left(\Delta \nabla_{t} \Omega_{1} \nabla^{t} \phi_{1}\right) .
$$

Now, we consider the silly divergence formula applied to (2-20) obtained by integrating by parts with respect to the function $\Omega_{1}$. If we denote the integrand in (2-20) by $F_{g}$, we denote the resulting (local) equation by silly $\left[F_{g}\right]=0$. We consider the sublinear combination silly* $\left[F_{g}\right]$ which consists of terms with length $\sigma+u, \mu$ internal contractions and $u-1+\beta$ factors $\nabla \phi_{h}, h \geq 2$, and a factor $\Delta \phi_{u+1}$. Clearly, this sublinear combination must vanish separately modulo longer terms,

$$
\operatorname{silly}^{*}\left[F_{g}\right]=0 .
$$

The above equation can be expressed as

$$
\operatorname{Spread}^{\nabla^{s}, \nabla_{s}}\left[\sum_{l \in \bar{L}_{\mu, \sharp}} a_{l} \bar{C}_{g}^{l}\right] \cdot \Omega_{1} \cdot \Delta \phi_{u+1}=0 .
$$

$\left(\right.$ Here $\operatorname{Spread}^{\nabla^{s}}, \nabla_{s}$ is a formal operation that acts on complete contractions in the form (1-5) by hitting a factor $T$ in the form $\nabla^{(m)} R_{i j k l}$ or $\nabla^{(p)} \Omega_{h}$ with a derivative $\nabla^{s}$ and then hitting another factor $T^{\prime} \neq T$ in the form $\nabla^{(m)} R_{i j k l}$ or $\nabla^{(p)} \Omega_{h}$ with a derivative $\nabla_{s}$ that contracts against $\nabla^{s}$ and then adding over all the terms we can thus obtain.) Now, using the fact that (2-21) holds formally, we derive $\mathrm{e}^{31}$

$$
\sum_{l \in L_{\mu, \sharp}} a_{l} \bar{C}_{g}^{l}=0 .
$$

Thus, applying the operation $S u b_{v} \mu-1$ times to the above and then multiplying by $\nabla_{i_{1} i_{2}} \Omega_{1} \nabla^{i_{1}} v \nabla^{i_{2}} \phi_{u+1}$ we derive (2-19). So for the rest of this proof we may assume that $\bar{L}_{\mu, \sharp}=\varnothing$.

Now we prove our claim under the additional assumption that for the tensor fields indexed in $\bar{L}_{\mu}$, the factor $\nabla^{(2)} \Omega_{1}$ contains no free index.

We again refer to (2-20) and perform integrations by parts with respect to the factor $\nabla^{(B)} \Omega_{1}$. We denote the resulting local equation by silly $\left[L_{g}\right]=0$. We pick out the sublinear combination silly* $\left[L_{g}\right]$ of terms with $\sigma+u$ factors, $u+\beta$ factors $\nabla \phi_{h}, \mu$ internal contractions, with $u+\beta-1$ factors $\nabla \phi_{h}, h \geq 2$, and a factor $\Delta \phi_{1}$. This sublinear combination must vanish separately, silly ${ }^{*}\left[L_{g}\right]=0$; the resulting new true equation can be described easily: Let us denote by $\hat{C}_{g}^{l, j_{1}}$ the 1 -vector

${ }^{31}$ This can be proven by using the operation Erase[... ], see the Appendix in [A 2010]. 
field that arises from $C_{g}^{l, i_{1} \ldots i_{\mu}}, l \in L_{\mu, *}$ by formally erasing the factor $\nabla_{j s}^{(2)} \Omega_{1} \nabla^{s} \phi_{1}$, making the index ${ }^{j}$ that contracted against ${ }_{j}$ into a free index ${ }_{j_{1}}$, and making all the free indices $i_{1}, \ldots, i_{\mu}$ into internal contractions. (Denote by $\vec{\kappa}_{\text {simp }}^{\prime}$ the simple character of these vector fields.) Then the equation silly* $\left[L_{g}\right]=0$ can be expressed in the form

$$
\sum_{l \in L_{\mu, *}} a_{l}\left\{X \operatorname{div}_{j_{1}} \hat{C}_{g}^{l, j_{1}}\right\} \Delta \phi_{1}+\sum_{j \in J} a_{j} C_{g}^{j} \Delta \phi_{1}=0
$$

here the complete contractions $C_{g}^{j}$ are simply subsequent to $\vec{\kappa}_{\text {simp }}^{\prime}$. The above holds modulo terms of length $\geq \sigma+u+1$. Now, we apply the operation $\operatorname{Sub}_{\omega} \mu$ times (see the Appendix in [A 2012]). In the case $\sigma>3$, we apply the inductive assumption of our Lemma 2.6 to the resulting equation (notice that the above falls under the inductive assumption of this lemma since we have lowered the weight in absolute value); we ensure that Lemma 2.6 can be applied by just labeling one of the factors $\nabla \omega$ into $\nabla \phi_{u+1}$. We derive (due to weight considerations) that there can not be tensor fields of higher rank, thus

$$
\sum_{l \in L_{\mu}} a_{l} \operatorname{Sub}_{\omega}^{\mu-1}\left[\hat{C}_{g}^{l, j_{1}}\right] \nabla_{i_{1}} v \Delta \phi_{1}=0
$$

Now, formally replacing the factor $\nabla_{i_{1}} v$ by $\nabla_{j_{1} t}^{(2)} \Omega_{1} \nabla^{t} \phi_{1}$, and then setting $\omega=v$, we derive the claim of our lemma. In the case $\sigma=3$ (2-24) follows by inspection, since the only two possible cases are $\sigma_{2}=2$ and $\sigma_{1}=2$; in the first case there are only two possible partial contractions in $\bar{L}_{\mu}$ while in the second there are four. Equation (2-23) (by inspection) implies that the coefficients of all these tensor fields must vanish, which is equivalent to (2-24).

Now, we will prove our claim under the additional assumption $\bar{L}_{\mu}=\varnothing$ (still for $p>0$ ). We again refer to (2-20) and again consider the same equation silly $\left[L_{g}\right]=0$ as above. We now pick out the sublinear combination of terms with $\sigma+u$ factors, $u+\beta$ factors $\nabla \phi_{h}$, and $\mu$ internal contractions. We derive that

$$
\sum_{l \in L_{\mu}} a_{l} X \operatorname{div}_{j_{1}} X \operatorname{div}_{j_{2}} \hat{C}_{g}^{l, j_{1} j_{2}}+\sum_{j \in J} a_{j} C_{g}^{j}=0
$$

here the terms $\hat{C}_{g}^{l, j_{1} j_{2}}$ arise from the $\mu$-tensor fields $C_{g}^{l i_{1} \ldots i_{\mu}}$ by replacing all $\mu$ free indices by internal contractions, erasing the factor $\nabla_{j k}^{(2)} \Omega_{1}$ and making the indices ${ }^{j},{ }^{k}$ into free indices ${ }^{j_{1}},{ }^{j_{2}}$. Now, applying $\operatorname{Sub}_{\omega} \mu$ times, and then applying the inductive assumption of Lemma 4.10 in [A 2010] (this applies by length considerations as above for $\sigma>3$; while if $\sigma=3$ the claim (2-26) will again follow by 
inspection) we derive that

$$
\sum_{l \in L_{\mu}} a_{l} \hat{C}_{g}^{l, j_{1} j_{2}} \nabla_{j_{1}} v \nabla_{j_{2}} v=0
$$

Replacing the expression $\nabla_{j_{1}} v \nabla_{j_{2}} v$ by a factor $\nabla_{j_{1} j_{2}}^{(2)} \Omega_{2}$ and then setting $\omega=v$, we derive our claim in the case $p>0$.

The case $p=0, \sigma_{1}>0$. We will reduce to the previous case: We let $L_{\mu}^{1}$ be the index set of $\mu$-tensor fields where the factor $T_{1}=S_{*} R_{i j k l} \nabla^{i} \tilde{\phi}_{1}$ contains a special free index (say the index ${ }_{k}$ is the free index $i_{\beta+1}$ without loss of generality). We will prove our claim for the index set $L_{\mu}^{1}$; if we can prove this, then clearly our lemma will follow by induction.

To prove this claim, we consider the first conformal variation of our hypothesis, Image $_{Y}^{1}\left[L_{g}\right]=0$, and we pick out the sublinear combination of terms with length $\sigma+u+\beta$, where the factor $\nabla^{(v)} S_{*} R_{i j k l} \nabla^{i} \tilde{\phi}_{1}$ has been replaced by a factor $\nabla^{(v+2)} Y$, and the factor $\nabla \phi_{1}$ now contracts against a factor $T_{2}=R_{i j k l}$. This sublinear combination vanishes separately, thus we derive a new local equation. To describe the resulting equation, we denote by

$$
\hat{C}_{g}^{l, i_{1} \ldots \hat{i}_{\beta+1} \ldots i_{\mu+\beta}}\left(Y, \phi_{1}, \ldots, \phi_{u}\right) \nabla_{i_{1}} \phi_{u+1} \ldots \nabla_{i_{\beta}} \phi_{u+\beta}
$$

the $(\mu-1)$-tensor field that arises from

$$
C_{g}^{l, i_{1} \ldots \hat{i}_{\beta+1} \ldots i_{\mu+\beta}}\left(Y, \phi_{1}, \ldots, \phi_{u}\right) \nabla_{i_{1}} \phi_{u+1} \ldots \nabla_{i_{\beta}} \phi_{u+\beta}
$$

by formally replacing the factor $T_{1}=S_{*} R_{i j k l} \nabla^{i} \tilde{\phi}_{1}$ by $\nabla_{j l}^{(2)} Y$ and also adding a derivative index $\nabla_{i_{*}}$ onto the factor $T_{2}=R_{i j k l}$ and then contracting that index $i_{*}$ against an (added anew) factor $\nabla \phi_{1}$. Denote the $(u-1)$-simple character of the above (the one defined by $\nabla \phi_{2}, \ldots, \nabla \phi_{u}$ ) by $\vec{\kappa}_{\text {simp }}^{\prime}$. We then have an equation

$$
\begin{aligned}
& \sum_{l \in L_{\mu}^{1}} a_{l} X \operatorname{div}_{i_{\beta+2}} \ldots X \operatorname{div}_{i_{\beta+\mu}} \hat{C}_{g}^{l, i_{1} \ldots \hat{i}_{\beta+1} \ldots i_{\mu+\beta}}\left(Y, \phi_{1}, \ldots, \phi_{u}\right) \nabla_{i_{1}} \phi_{u+1} \ldots \nabla_{i_{\beta}} \phi_{u+\beta} \\
& \quad+\sum_{h \in H} a_{h} X \operatorname{div}_{i_{\beta+1}} \ldots X \operatorname{div}_{i_{\mu+\beta}} C_{g}^{l, i_{2} \ldots i_{\mu+\beta}}\left(Y, \phi_{1}, \ldots, \phi_{u}\right) \nabla_{i_{1}} \phi_{u+1} \ldots \nabla_{i_{\beta}} \phi_{u+\beta} \\
& =\sum_{j \in J} a_{j} C_{g}^{j, i_{\mu+1} \ldots i_{\beta}}\left(Y, \phi_{1}, \ldots, \phi_{u}\right) \nabla_{i_{\mu+1}} \phi_{u+1} \ldots \nabla_{i_{\beta}} \phi_{u+\beta} .
\end{aligned}
$$

The terms indexed in $H$ are acceptable, have a $(u-1)$-simple character $\vec{\kappa}_{\text {simp }}^{\prime}$ and the factor $\nabla \phi_{1}$ contracts against an internal index (without loss of generality, say the index ${ }_{i}$ in the factor $T_{2}=R_{i j k l}$ ); writing that factor as $S_{*} R_{i j k l} \nabla^{i} \tilde{\phi}_{1}$, we denote the resulting $u$-simple factor by $\tilde{\kappa}_{\text {simp. }}$. The terms indexed in $J$ are simply subsequent 
to $\vec{\kappa}_{\text {simp }}^{\prime}$. Now, applying the inductive assumption of Lemma $2.6,{ }^{32}$ we derive that

$$
\begin{aligned}
\sum_{h \in H} a_{h} C_{g}^{l, i_{2} \ldots i_{\mu+\beta}}\left(Y, \phi_{1}, \ldots\right. & \left., \phi_{u}\right) \\
& \times \nabla_{i_{1}} \phi_{u+1} \ldots \nabla_{i_{\beta+1}} \phi_{u+\beta} \nabla_{i_{\beta+1}} v \ldots \nabla_{i_{\beta+\mu}} v=0 .
\end{aligned}
$$

Thus, we may assume without loss of generality that $H=\varnothing$ in (2-27). Now, we again apply Lemma 2.6 to (2-27) (under that additional assumption), and we derive that

$$
\begin{aligned}
\sum_{l \in L_{\mu}^{1}} a_{l} \hat{C}_{g}^{l, i_{1} \ldots \hat{i}_{\beta+1} \ldots i_{\mu+\beta}}\left(Y, \phi_{1}\right. & \left.\ldots, \phi_{u}\right) \\
& \times \nabla_{i_{1}} \phi_{u+1} \ldots \nabla_{i_{\beta}} \phi_{u+\beta} \nabla_{i_{\beta+1}} v \ldots \nabla_{i_{\beta+\mu}} v=0 .
\end{aligned}
$$

Now, erasing the factor $\nabla \phi_{1}$ from the above, and then formally replacing the factor $\nabla_{a b}^{(2)} Y$ by $S_{*} R_{i(a b) l} \nabla^{i} \tilde{\phi}_{1} \nabla^{l} v$, we derive our claim.

The case $p=0, \sigma_{1}=0$. In this case $\sigma=\sigma_{2}$. In other words, all factors in $\vec{\kappa}_{\text {simp }}$ are simple factors in the form $S_{*} R_{i j k l} \nabla^{i} \tilde{\phi}_{h}$. We recall that in this case all $\mu$-tensor fields in (2-9) must have at most one free index in any factor $S_{*} R_{i j k l}$. In that case, we will prove our claim in a more convoluted manner, again reducing ourselves to the inductive assumption of Proposition 2.1 in [A 2010].

A key observation is that by the definition of the special cases, $\mu+\beta \leq \sigma_{2}$. In the case of strict inequality, we see (by a counting argument) that at least one of the special indices in one of the factors $S_{*} R_{i j k l}$ must contract against a special index in another factor $S_{*} R_{a b c d}$. In the case $\mu+\beta=\sigma_{2}$ this remains true, except for the terms for which the $\beta$ factors $\nabla \phi_{u+h}$ contract against special indices, say the indices ${ }_{k}$, in $\beta$ factors $T_{y}=S_{*} R_{i k l} \nabla^{i} \tilde{\phi}_{y}$, and moreover these factors must not contain a free index, and all other factors $S_{*} R_{i k l}$ contain exactly one free index, which must be special. In this subcase, we will prove our claim for all $\mu$-tensor fields excluding this particular "bad" sublinear combination; we will prove our claim for this sublinear combination in the end.

We will now proceed to normalize the different $(\mu+\beta)$-tensor fields in (2-9). A normalized tensor field will be in the form (1-6), with possibly certain pairs of indices in certain of the factors $S_{*} R_{i j k l}$ being symmetrized over.

Let us first introduce a few definitions: Given each $C_{g}^{l, i_{1} \ldots i_{\mu}}$, we list out the factors $T_{1}, \ldots T_{\sigma_{2}}$ in the form $S_{*} R_{i k l}$. Here $T_{a}$ is the factor for which the index $i$ is contracting against the factor $\nabla \tilde{\phi}_{a}$. We say that factors $S_{*} R_{i k l}$ are of type I if they contain no free index. We say they are of type II if they contain a special free index. We say they are of type III if they contain a nonspecial free index.

Given any tensor field $C_{g}^{l, i_{1} \ldots i_{\mu}}$ in the form (1-6), pick out the pairs of factors $T_{\alpha}, T_{\beta}$ in the form $S_{*} R_{i j k l}$ for which a special index in $T_{\alpha}$ contracts against a special

${ }^{32}$ The terms indexed in $L_{\mu}^{1}$ are now simply subsequent to $\tilde{\kappa}_{\text {simp. }}$ 
index in $T_{\beta}$. (Call such particular contractions "special-to-special" particular contractions.) Now, in any $C_{g}^{l, i_{1} \ldots i_{\mu}}$ we define an ordering among all its factors $S_{*} R_{i j k l}$ : The factor $T_{a}=S_{*} R_{i k l} \nabla^{i} \tilde{\phi}_{a}$ is more important than $T_{b}=S_{*} R_{i^{\prime} j^{\prime} k^{\prime} l^{\prime}} \nabla^{i^{\prime}} \tilde{\phi}_{b}$ if $a<b$.

Now, consider a tensor field $C_{g}^{l, i_{1} \ldots i_{\mu}}$ and list out all the pairs of factors $T_{a}, T_{b}$ with a special-to-special particular contraction. We say that $\left(T_{a}, T_{b}\right)$ is the most important pair of factors with a special-to-special particular contraction ${ }^{33}$ if any other such pair $\left(T_{c}, T_{d}\right)^{34}$ has either $T_{c}$ less important than $T_{a}$ or $T_{a}=T_{c}$ and $T_{d}$ less important than $T_{b}$.

Now, consider a tensor field $C_{g}^{l, i_{1} \ldots i_{\mu}}$ and consider the most important pair of factors $\left(T_{a}, T_{b}\right)$ with a special-to-special particular contraction. Assume without loss of generality that the index $l$ in $T_{a}=S_{*} R_{i j k l} \nabla^{i} \tilde{\phi}_{a}$ contracts against the index $l^{\prime}$ in $T_{b}=S_{*} R_{i^{\prime} j^{\prime} k^{\prime} l^{\prime}} \nabla^{i^{\prime}} \tilde{\phi}_{b}$. We say that $C_{g}^{l, i_{1} \ldots i_{\mu}}$ is normalized if both factors $T_{a}, T_{b}$ are normalized. The factor $T_{a}=S_{*} R_{i k l} \nabla^{i} \tilde{\phi}_{a}$ is normalized if: Either the index ${ }_{j}$ contracts against a factor $T_{c}$ that is more important than $T_{b}$, or if the indices ${ }_{j},{ }_{k}$ are symmetrized. If $T_{a}$ is of type II, then we require that the index ${ }_{j}$ in $T_{b}=S_{*} R_{i j \text { (free)l }}$ must contract against a special index of some other factor $T_{c}$, and moreover $T_{c}$ must be more important than $T_{b}$. If $T_{a}$ is of type III, then it is automatically normalized. The same definition applies to $T_{b}$, where any reference to $T_{b}$ must be replaced by a reference to $T_{a}$.

Let us now prove that we may assume without loss of generality that all $\mu$ tensor fields in (2-9) are normalized: Consider a $C_{g}^{l, i_{1} \ldots i_{\mu}}$ in (2-9) for which the most important pair of factors with a special-to-special particular contraction is the pair $\left(T_{a}, T_{b}\right)$. We will prove that we can write

$$
C_{g}^{l, i_{1} \ldots i_{\mu+\beta}}=\tilde{C}_{g}^{l, i_{1} \ldots i_{\mu+\beta}}+\sum_{t \in T} a_{t} C_{g}^{t, i_{1} \ldots i_{\mu+\beta}}
$$

here the term $\tilde{C}_{g}^{l, i_{1} \ldots i_{\mu+\beta}}$ is normalized, the most important pair of factors with a special-to-special particular contraction is the pair $\left(T_{a}, T_{b}\right)$, and moreover its refined double character is the same as for $C_{g}^{l, i_{1} \ldots i_{\mu+\beta}}$. Each term $C_{g}^{t, i_{1} \ldots i_{\mu+\beta}}$ has either the same, or a doubly subsequent refined double character to $C_{g}^{l, i_{1} \ldots i_{\mu+\beta}}$; moreover in the first case its most important pair of factors with a special-to-special particular contraction will be less important than the pair $\left(T_{a}, T_{b}\right)$. In the second case the most important pair will either be $\left(T_{a}, T_{b}\right)$ or a less important pair.

Clearly, if we can prove the above, then by iterative repetition we may assume without loss of generality that all $(\mu+\beta)$-tensor fields in (2-9) are normalized.

Proof of (2-30). Pick out the most important pair of factors with a special-tospecial particular contraction is the pair $\left(T_{a}, T_{b}\right)$ in $C_{g}^{l, i_{1} \ldots i_{\mu+\beta}}$. Let us first normalize

${ }^{33}$ Assume without loss of generality that $T_{a}$ is more important than $T_{b}$.

${ }^{34}$ Again assume without loss of generality that $T_{c}$ is more important than $T_{d}$. 
$T_{a}$. If $T_{a}$ is of type III, there is nothing to do. If it is of type II and already normalized, there is again nothing to do. If it is of type II and not normalized, then we interchange the indices ${ }_{j}, k$. The resulting factor is normalized. The correction term we obtain by virtue of the first Bianchi identity is also normalized (it is of type III). Moreover, the resulting tensor field is doubly subsequent to $C_{g}^{l, i_{1} \ldots i_{\mu+\beta}}$. Finally, if the factor $T_{a}$ is of type I, we inquire on the factor $T_{c}$ against which ${ }_{j}$ in $T_{a}=S_{*} R_{i j k l}$ contracts: If it is more important than $T_{b}$, then we leave $T_{a}$ as it is; it is already normalized. If not, we symmetrize ${ }_{j}, k$. The resulting tensor field is normalized. The correction term we obtain by virtue of the first Bianchi identity will then have the same refined double character as $C_{g}^{l, i_{1} \ldots i_{\mu+\beta}}$, and moreover its most important pair of factors with a special-to-special particular contraction is less important than that pair $\left(T_{a}, T_{b}\right)$.

We may now prove the claim of Lemma 2.6 in this special case, under the additional assumption that all tensor fields in (2-9) are normalized. We list out the most important pair of special-to-special particular contractions in each $C_{g}^{l, i_{1} \ldots i_{\mu+\beta}}$, and denote it by $(a, b)_{l}$. We let $(\alpha, \beta)$ stand for the lexicographically minimal pair among the list $(a, b)_{l}, l \in L_{\mu}$. We denote by $L_{\mu}^{(\alpha, \beta)} \subset L_{\mu}$ the index set of terms with a special-to-special particular contraction among the terms $T_{\alpha}, T_{\beta}$. We will prove that

$$
\sum_{l \in L_{\mu}^{(\alpha, \beta)}} a_{l} C_{g}^{l, i_{1} \ldots i_{\mu+\beta}} \nabla_{i_{1}} v \ldots \nabla_{i_{\mu}} v=0 .
$$

Clearly, the above will imply our claim, by iterative repetition. ${ }^{35}$

Proof of (2-31). Consider Image $e_{Y_{1}, Y_{2}}^{2}\left[L_{g}\right]=0$ and pick out the sublinear combination where the factors $T_{\alpha}, T_{\beta}$ are replaced by $\nabla^{(A)} Y_{1} \otimes g, \nabla^{(B)} Y_{2} \otimes g$, and the two factors $\nabla \tilde{\phi}_{\alpha}, \nabla \tilde{\phi}_{\beta}$ contract against each other. The resulting sublinear combination must vanish separately. We erase the expression $\nabla_{t} \tilde{\phi}_{\alpha} \nabla^{t} \tilde{\phi}_{\beta},{ }^{36}$ and derive a new true equation in the form

$$
\sum_{l \in L_{\mu}^{(\alpha, \beta)}} a_{l} X \operatorname{div}_{i_{1}} \ldots X \operatorname{div}_{i_{\mu}} \tilde{C}_{g}^{l, i_{1} \ldots i_{\mu+\beta}}\left(\Omega_{1}, Y_{1}, Y_{2}\right)+\sum_{j \in J} a_{j} C_{g}^{j}\left(\Omega_{1}, Y_{1}, Y_{2}\right)=0
$$

here the tensor fields $\tilde{C}_{g}^{l, i_{1} \ldots i_{\mu+\beta}}\left(\Omega_{1}, Y_{1}, Y_{2}\right)$ arise from the tensor fields $C_{g}^{l, i_{1} \ldots i_{\mu+\beta}}$ by replacing the expression $\nabla^{i} \tilde{\phi}_{\alpha} S_{*} R_{i j k l} \otimes S_{*} R_{i^{\prime} j k}^{l} \nabla^{i^{\prime}} \tilde{\phi}_{\beta}$ with $\nabla_{j k} Y_{1} \otimes \nabla_{j^{\prime} k^{\prime}} Y_{2}$ (notice we have lowered the weight in absolute value).

${ }^{35}$ In the subcase $\mu+\beta=\sigma_{2}$ it will only imply it for the "excluded" sublinear combination defined above.

${ }^{36}$ Denote the resulting $(u-2)$-simple character by $\vec{\kappa}_{\text {simp }}^{\prime \prime \prime}$. 
Now, applying the inductive assumption of Lemma 2.6 to the above, ${ }^{37}$ we derive

$$
\sum_{l \in L_{\mu}^{(\alpha, \beta)}} a_{l} \tilde{C}_{g}^{l, i_{1} \ldots i_{\mu+\beta}}\left(\Omega_{1}, Y_{1}, Y_{2}\right) \nabla_{i_{1}} v \ldots \nabla_{i_{\mu}} v=0 .
$$

The proof of (2-31) is only one step away. Let us start with an important observation: For each given complete contraction above, examine the factor $\nabla_{z x}^{(2)} Y_{1}$; it either contracts against no factor $\nabla v$ or against one factor $\nabla v{ }^{38}$ In the first case, the factor $\nabla_{z x}^{(2)} Y_{1}$ must have arisen from a factor $S_{*} R_{i j k l}$ of type I. In fact, the indices $z,{ }_{x}$ correspond to the indices ${ }_{j},{ }_{k}$ in the original factor, and we can even determine their position: Since the pair $(\alpha, \beta)$ is the most important pair in (2-9), at most one of the indices ${ }_{z}, x$ can contract against a special index in a more important factor than $T_{\beta}$. If one of them does ( say $z$ ), then that index must have been the index ${ }_{j}$ in $T_{\alpha}=S_{*} R_{i k l}$. If none of them does, then the two indices ${ }_{z},{ }_{x}$ must be symmetrized over, since the two indices ${ }_{j},{ }_{k}$ in $T_{\alpha}$ to which they correspond were symmetrized over. Now, these two separate sublinear combinations in (2-33) must vanish separately (this can be proven using the eraser from the Appendix in [A 2012]), and furthermore in the first case, we may assume that the index $z_{z}$ (which contracts against a special index in a more important factor than $T_{\beta}$ ) occupies the leftmost position in $\nabla_{z x}^{(2)} Y_{1}$ and is not permuted in the formal permutations of indices that make (2-33) hold formally).

On the other hand, consider the terms in (2-33) with the factor $\nabla^{(2)} Y_{1}$ contracting against a factor $\nabla v$. By examining the index ${ }_{y}$ in the factor $\nabla_{y t}^{(2)} Y_{1} \nabla^{t} v$, we can determine the type of factor in $C_{g}^{l, i_{1} \ldots i_{\mu+\beta}}$ from which the factor $\nabla^{(2)} Y_{1}$ arose: If the index $y$ contracts against a special index in a factor $S_{*} R_{i j k l}$ which is more important than $T_{\beta}$, then $\nabla^{(2)} Y_{1}$ can only have arisen from a factor of type II in $C_{g}^{l, i_{1} \ldots i_{\mu+\beta}}$. In fact, the index ${ }_{y}$ in $\nabla^{(2)} Y_{1}$ must correspond to the index ${ }_{j}$ in $S_{*} R_{i j(\text { free }) l}$ in $T_{\alpha}$. If the index $y$ in $\nabla_{y t}^{(2)} Y_{1} \nabla^{t} v$ does not contract against a special index in a factor $T_{c}$ which is more important than $T_{\beta}$, then the factor $\nabla^{(2)} Y_{1}$ can only have arisen from a factor of type III in $C_{g}^{l, i_{1} \ldots i_{\mu+\beta}}$. In fact, the index ${ }_{y}$ in $\nabla^{(2)} Y_{1}$ must correspond to the index ${ }_{k}$ in $S_{*} R_{i(\text { free }) k l}$ in $T_{\alpha}$.

The same analysis can be repeated for the factor $\nabla^{(2)} Y_{2}$, with any reference to the factor $T_{\beta}$ now replaced by the factor $T_{\alpha}$.

In view of the above analysis, we can break the left-hand side of (2-33) into four sublinear combinations that vanish separately (depending on whether $\nabla^{(2)} Y_{1}$, $\nabla^{(2)} Y_{2}$ contract against a factor $\nabla v$ or not). Then in each of the four sublinear combinations, we can arrange that in the formal permutations that make the lefthand side of (2-33) formally zero, the two indices in the factors $\nabla^{(2)} Y_{1}, \nabla^{(2)} Y_{2}$ are

${ }^{37}$ We have lowered the weight in absolute value.

${ }^{38}$ The two corresponding sublinear combinations vanish separately, of course. 
not permuted (by virtue of the remarks above). In view of this and the analysis in the previous paragraph, we can then replace the two factors $\nabla_{z x}^{(2)} Y_{1}, \nabla_{q w}^{(2)} Y_{2}$ by an expression $\nabla^{i} \tilde{\phi}_{\alpha} S_{*} R_{i z x l} \otimes S_{*} R_{i^{\prime} q w}{ }^{l} \nabla^{i^{\prime}} \tilde{\phi}_{\beta}$, in such a way that the resulting linear combination vanishes formally without permuting the two indices $q, w, q^{\prime}, w^{\prime}$. This proves our claim, except for the subcase $\mu+\beta=\sigma_{2}$ where we only derive our claim for all terms except for the "bad sublinear combination". We now prove our claim for that case.

The "bad sublinear combination". We break up the left-hand side of (2-16) according to which factor $T_{s}$ the factor $\nabla \phi_{u+1}$ contracts - denote the index set of those terms by $L_{\mu}^{K}$. Denote the resulting sublinear combinations by $L_{g}^{K}, K=1, \ldots, \sigma_{2}$. Given any $K$, we consider the equation $\operatorname{Image}_{Y}^{1}\left[L_{g}\right]=0$, and we pick out the sublinear combination where the term $\nabla^{(B)} S_{*} R_{i j k l} \nabla^{i} \tilde{\phi}_{K}$ is replaced by $\nabla^{(B+2)} Y$, and the factor $\nabla \phi_{K}$ now contracts against the factor $\nabla \phi_{u+1}$. This sublinear combination must vanish separately. We then again perform the "inverse integration by parts" to this true equation (deriving an integral equation), and then we consider the silly divergence formula for this integral equation, obtained by integrating by parts with respect to $\nabla^{(B)} Y$. We pick out the sublinear combination with $\sigma+u+\beta$ factors, $\mu$ internal contractions and $u+\beta$ factors $\nabla \phi_{h}$, and an expression $\nabla_{s} \phi_{u+1} \nabla^{s} \tilde{\phi}_{K}$ This gives us a new true local equation,

$$
\sum_{l \in L_{\mu}^{K}} a_{l} X_{*} \operatorname{div}_{j_{1}} X_{*} \operatorname{div}_{j_{2}} \tilde{C}_{g}^{l, j_{1} j_{2}}+\sum_{j \in J} a_{j} C_{g}^{j}=0 .
$$

Here the tensor fields $\tilde{C}_{g}^{l, j_{1} j_{2}}$ arise from $C_{g}^{l, i_{1} \ldots i_{\mu}}$ by formally replacing all $\mu$ free indices with internal contractions, and also replacing $\nabla_{x} \phi_{u+1} \otimes S_{*} R_{i(j k)}{ }^{x} \nabla^{i} \tilde{\phi}_{K}$ with $\nabla_{x} \phi_{u+1} \nabla^{s} \tilde{\phi}_{K} \otimes Y$, and then making the indices ${ }^{j},{ }^{k}$ that contracted against ${ }_{j},{ }_{k}$ into free indices ${ }^{j_{1}},{ }^{j_{2}} . X_{*} \operatorname{div}_{j}$ stands for the sublinear combination in $X \operatorname{div}_{j}$ where $\nabla^{j}$ is not allowed to hit the factor $Y$. Now, applying the inductive assumption of Lemma 2.6 to the above, ${ }^{39}$ we derive that

$$
\sum_{l \in L_{\mu}^{K}} a_{l} \tilde{C}_{g}^{l, j_{2}} \nabla_{j_{1}} \omega \nabla_{j_{2}} \omega=0
$$

We replace $\nabla^{x} \phi_{K} \nabla_{x} \phi_{u+1} \nabla_{j_{1}} \omega \nabla_{j_{2}} \omega \nabla_{l} Y$ with $\nabla^{l} \phi_{u+1} S_{*} R_{i\left(j_{1} j_{2}\right) l} \nabla^{i} \tilde{\phi}_{K}$ and then replace all internal contractions by factors $\nabla v$ (applying the operation $\operatorname{Sub}_{v}$ from the Appendix in [A 2012]). The resulting (true) equation is precisely our remaining claim for the "bad" sublinear combination.

${ }^{39} \mathrm{We}$ have lowered the weight in absolute value. 
2C. Proof of Lemmas 4.6, 4.8 in [A 2010]: The main part. We first write down the form of the complete and partial contractions that we are dealing with in Lemmas 2.1 and 2.3. In the setting of Lemma 2.1 we recall that the tensor fields $C^{h, i_{1} \ldots i_{\alpha}}$ indexed in $\mathrm{H}_{2}$ (in the hypothesis of Lemma 2.1) are all partial contractions in the form

$$
\begin{aligned}
\operatorname{pcontr}\left(\nabla^{\left(m_{1}\right)} R_{i j k l} \otimes \cdots \otimes \nabla^{\left(m_{\sigma_{1}}\right)} R_{i j k l} \otimes S_{*} \nabla^{\left(v_{1}\right)} R_{i j k l} \otimes \cdots \otimes S_{*} \nabla^{\left(v_{t}\right)} R_{i j k l}\right. \\
\otimes \nabla^{\left(b_{1}\right)} \Omega_{1} \otimes \cdots \otimes \nabla^{\left(b_{p}\right)} \Omega_{p} \otimes \nabla Y \otimes \nabla \phi_{z_{1}} \cdots \otimes \nabla \phi_{z_{f}} \\
\left.\otimes \nabla \phi_{z_{f+1}}^{\prime} \otimes \cdots \otimes \nabla \phi_{z_{f+d}}^{\prime} \otimes \cdots \otimes \nabla \tilde{\phi}_{z_{f+d+1}} \otimes \cdots \otimes \nabla \tilde{\phi}_{z_{f+d+y}}\right),
\end{aligned}
$$

where we let $f+d+y=u^{\prime}$. The main assumption here is that all tensor fields have the same $u^{\prime}$-simple character (the one defined by $\nabla \phi_{1}, \ldots, \nabla \phi_{u^{\prime}}$ ), which we denote by $\vec{\kappa}_{\text {simp }}^{+}$. The other main assumption is that if we formally treat the factor $\nabla Y$ as a function $\nabla \phi_{u+1}$, then the hypothesis of Lemma 2.1 falls under the inductive assumptions of Proposition 1.1 (i.e., the weight, real length, $\Phi$ and $p$ are as in our inductive assumption of Proposition 1.1).

In the setting of Lemma 2.3 we recall that we are dealing with complete and partial contractions in the form

$$
\begin{gathered}
\operatorname{contr}\left(\nabla^{\left(m_{1}\right)} R_{i j k l} \otimes \cdots \otimes \nabla^{\left(m_{\sigma_{1}}\right)} R_{i j k l} \otimes S_{*} \nabla^{\left(v_{1}\right)} R_{i j k l} \otimes \cdots \otimes S_{*} \nabla^{\left(v_{t}\right)} R_{i j k l}\right. \\
\otimes \nabla^{\left(b_{1}\right)} \Omega_{1} \otimes \cdots \otimes \nabla^{\left(b_{p}\right)} \Omega_{p} \otimes\left[\nabla \omega_{1} \otimes \nabla \omega_{2}\right] \otimes \nabla \phi_{z_{1}} \cdots \otimes \nabla \phi_{z_{f}} \\
\left.\otimes \nabla \phi_{z_{f+1}^{\prime}}^{\prime} \otimes \cdots \otimes \nabla \phi_{z_{f+d}^{\prime}}^{\prime} \otimes \cdots \otimes \tilde{\phi}_{z_{f+d+1}} \otimes \cdots \otimes \tilde{\phi}_{z_{f+d+y}}\right),
\end{gathered}
$$

where we let $f+d+y=u^{\prime}$. The main assumption here is that all partial contractions have the same $u^{\prime}$-simple character (the one defined by $\nabla \phi_{1}, \ldots, \nabla \phi_{u^{\prime}}$ ), which we denote by $\vec{\kappa}_{\text {simp }}^{+}$. The other main assumption is that if we formally treat the factors $\nabla \omega_{1}, \nabla \omega_{2}$ as factors $\nabla \phi_{u+1}, \nabla \phi_{u+2}$, then the hypothesis of Lemma 2.3 falls under the inductive assumptions of Proposition 1.1 (i.e., the weight, real length, $\Phi$ and $p$ are as in our inductive assumption of Proposition 1.1).

Note. From now on, we will be writing $u^{\prime}=u$, for simplicity. We will also be writing $\vec{\kappa}_{\text {simp }}^{+}=\vec{\kappa}_{\text {simp }}$, for simplicity. We will also be labeling the indices $i_{1}, \ldots, i_{\alpha}$ as $i_{\pi+1}, \ldots, i_{\alpha+1}$.

New induction. We will now prove the two Lemmas 2.1 and 2.3 by a new induction on the weight of the complete contractions in the hypotheses of those lemmas. We will assume that these two lemmas are true when the weight of the complete contractions in their hypotheses is $-W$, for any $W<K \leq n$. We will then show our lemmas for weight $-K$.

Reduce Lemma 2.1 to two lemmas. In order to show Lemma 2.1, we further break up $H_{2}$ into subsets: We say that $h \in H_{2}^{a}$ if $C^{h, i_{\pi+1} \ldots i_{\alpha+1}}$ has a free index (say the free index $i_{\alpha+1}$ without loss of generality) belonging to the factor $\nabla Y$. On the other 
hand, we say that $h \in H_{2}^{b}$ if the index in the factor $\nabla Y$ is not free. Lemma 2.1 will then follow from Lemmas 2.7 and 2.8 below.

Lemma 2.7. There exists a linear combination of acceptable $(\alpha-\pi+1)$-tensor fields, $\sum_{v \in V} a_{v} C_{g}^{v, i_{\pi+1} \ldots i_{\alpha+2}}\left(\Omega_{1}, \ldots, \Omega_{p}, Y, \phi_{1}, \ldots, \phi_{u}\right)$, where the index $i_{\alpha+1} b e$ longs to the factor $\nabla Y$, with a simple character $\vec{\kappa}_{\text {simp }}$, so that

$$
\begin{aligned}
& \sum_{h \in H_{2}^{a}} a_{h} C_{g}^{h, i_{\pi+1} \ldots i_{\alpha+1}}\left(\Omega_{1}, \ldots, \Omega_{p}, Y, \phi_{1}, \ldots, \phi_{u}\right) \nabla_{i_{\pi+1}} v \ldots \nabla_{i_{\alpha+1}} v \\
& =\sum_{v \in V} a_{v} X_{*} \operatorname{div}_{i_{\alpha+2}} C_{g}^{v, i_{\pi+1} \ldots i_{\alpha+2}}\left(\Omega_{1}, \ldots, \Omega_{p}, Y, \phi_{1}, \ldots, \phi_{u}\right) \\
& \quad \times \nabla_{i_{\pi+1}} v \ldots \nabla_{i_{\alpha+1}} v \\
& \quad+\sum_{j \in J} a_{j} C_{g}^{j, i_{\pi+1} \ldots i_{\alpha+1}}\left(\Omega_{1}, \ldots, \Omega_{p}, Y, \phi_{1}, \ldots, \phi_{u}\right) \nabla_{i_{\pi+1}} v \ldots \nabla_{i_{\alpha+1}} v .
\end{aligned}
$$

Each $C^{j}$ is simply subsequent to $\vec{\kappa}_{\text {simp. }}$

We observe that if we can show our first claim, then we can assume, with no loss of generality, that $H_{2}^{a}=\varnothing$, since it immediately follows from the above that

$$
\begin{aligned}
& \sum_{h \in H_{2}^{a}} a_{h} X_{*} \operatorname{div}_{i_{\pi+1}} \ldots X_{*} \operatorname{div}_{i_{\alpha+1}} C_{g}^{h, i_{\pi+1} \ldots i_{\alpha+1}}\left(\Omega_{1}, \ldots, \Omega_{p}, Y, \phi_{1}, \ldots, \phi_{u}\right) \\
& =\sum_{v \in V} a_{v} X_{*} \operatorname{div}_{i_{\pi+1}} \ldots X_{*} \operatorname{div}_{i_{\alpha+1}} X_{*} \operatorname{div}_{i_{\alpha+2}} \\
& \quad \times C_{g}^{v, i_{\pi+1} \ldots i_{\alpha+2}}\left(\Omega_{1}, \ldots, \Omega_{p}, Y, \phi_{1}, \ldots, \phi_{u}\right) \\
& \quad+\sum_{j \in J} a_{j} C_{g}^{j}\left(\Omega_{1}, \ldots, \Omega_{p}, Y, \phi_{1}, \ldots, \phi_{u}\right),
\end{aligned}
$$

where each complete contraction $C^{j}$ is subsequent to $\vec{\kappa}_{\text {simp. }}$. Note that one of the free indices in the tensor fields $C_{g}^{v, i_{\pi+1} \ldots i_{\alpha+2}}$ will belong to the factor $\nabla Y$.)

The second claim, in the setting of Lemma 2.1 is:

Lemma 2.8. We assume $H_{2}^{a}=\varnothing$. We then claim that modulo complete contractions of length $\geq \sigma+u+1$,

$$
\begin{gathered}
\sum_{h \in H_{2}} a_{h} C_{g}^{h, i_{\pi+1} \ldots i_{\alpha+1}}\left(\Omega_{1}, \ldots, \Omega_{p}, Y, \phi_{1}, \ldots, \phi_{u}\right) \nabla_{i_{\pi+1}} v \ldots \nabla_{i_{\alpha+1}} v \\
=\sum_{t \in T} a_{t} X_{*} \operatorname{div}_{i_{\alpha+2}} C_{g}^{t, i_{\pi+1} \ldots i_{\alpha+2}}\left(\Omega_{1}, \ldots, \Omega_{p}, Y, \phi_{1}, \ldots, \phi_{u}\right) \\
\quad+\sum_{j \in J} a_{j} C_{g}^{j}\left(\Omega_{1}, \ldots, \Omega_{p}, Y, \phi_{1}, \ldots, \phi_{u}\right), \\
\quad
\end{gathered}
$$

where each $C^{j}$ is acceptable and subsequent to $\vec{\kappa}_{\text {simp }}$. 
We observe that if we can show the above two lemmas then Lemma 2.1 will follow. (Notice that replacing by the right-hand side of (2-38) into the hypothesis of Lemma 2.1, we do not introduce 1-forbidden terms.)

We make two analogous claims for Lemma 2.3:

Reduce Lemma 2.3 to two lemmas. We say that $h \in H_{2}^{a}$ if $C^{h, i_{\pi+1} \ldots i_{\alpha+1}}$ has a free index belonging to one of the factors $\nabla \omega_{1}, \nabla \omega_{2}$. On the other hand, we say that $h \in H_{2}^{b}$ if none of the factors $\nabla \omega_{1}, \nabla \omega_{2}$ in $C^{h, i_{\pi+1} \ldots i_{\alpha+1}}$ contains a free index. (Observe that we may assume with no loss of generality that there are no tensor fields $C^{h, i_{\pi+1} \ldots i_{\alpha+1}}$ with free indices in both factors $\nabla \omega_{1}, \nabla \omega_{2}$ - this is by virtue of the antisymmetry of the factors $\nabla \omega_{1}, \nabla \omega_{2}$.) We make two claims. First:

Lemma 2.9. There is a linear combination of acceptable $(\alpha-\pi+1)$-tensor fields, $\sum_{v \in V} a_{v} C_{g}^{v, i_{\pi+1} \ldots i_{\alpha+2}}\left(\Omega_{1}, \ldots, \Omega_{p},\left[\omega_{1}, \omega_{2}\right], \phi_{1}, \ldots, \phi_{u}\right)$, in the form (2-35) with a simple character $\vec{\kappa}_{\text {simp }}$, so that

$$
\begin{aligned}
& \sum_{h \in H_{2}^{a}} a_{h} X_{+} \operatorname{div}_{i_{\pi+1}} \ldots X_{+} \operatorname{div}_{i_{\alpha+1}} \\
& \quad \times C_{g}^{h, i_{\pi+1} \ldots i_{\alpha+1}}\left(\Omega_{1}, \ldots, \Omega_{p},\left[\omega_{1}, \omega_{2}\right], \phi_{1}, \ldots, \phi_{u}\right) \\
& =\sum_{v \in V} a_{v} X_{+} \operatorname{div}_{i_{\pi+1}} \ldots X_{+} \operatorname{div}_{i_{\alpha+1}} X_{+} \operatorname{div}_{i_{\alpha+2}} \\
& \quad \times C_{g}^{v, i_{\pi+1} \ldots i_{\alpha+2}}\left(\Omega_{1}, \ldots, \Omega_{p},\left[\omega_{1}, \omega_{2}\right], \phi_{1}, \ldots, \phi_{u}\right) \\
& +\sum_{q \in Q} a_{q} X_{+} \operatorname{div}_{i_{\pi+1}} \ldots X_{+} \operatorname{div}_{i_{\alpha+1}} \\
& \quad \times C_{g}^{q, i_{\pi+1} \ldots i_{\alpha+1}}\left(\Omega_{1}, \ldots, \Omega_{p}, \nabla_{+}\left[\omega_{1}, \omega_{2}\right], \phi_{1}, \ldots, \phi_{u}\right) \\
& +\sum_{j \in J} a_{j} C_{g}^{j}\left(\Omega_{1}, \ldots, \Omega_{p},\left[\omega_{1}, \omega_{2}\right], \phi_{1}, \ldots, \phi_{u}\right) .
\end{aligned}
$$

(Recall that by definition the complete contractions indexed in $Q$ have a factor $\left.\nabla^{(2)} \omega_{1}.\right)$

We observe that if we can show our first claim, then we can, with no loss of generality, assume that $H_{2}^{a}=\varnothing$.

Second claim:

Lemma 2.10. We assume $H_{2}^{a}=\varnothing$, and that for some $k \geq 1$, we can write

$$
\begin{aligned}
& \sum_{h \in H_{2}^{b}} a_{x} X_{+} \operatorname{div}_{i_{\pi+1}} \ldots X_{+} \operatorname{div}_{i_{\alpha+1}} \\
& \times C_{g}^{h, i_{\pi+1} \ldots i_{\alpha+1}}\left(\Omega_{1}, \ldots, \Omega_{p},\left[\omega_{1}, \omega_{2}\right], \phi_{1}, \ldots, \phi_{u}\right) \\
&=\sum_{t \in T_{k}} a_{t} X_{+} \operatorname{div}_{i_{\pi+1}} \ldots X_{+} \operatorname{div}_{i_{\alpha+k}} \\
& \times C_{g}^{t, i_{\pi+1} \ldots i_{\alpha+k}}\left(\Omega_{1}, \ldots, \Omega_{p},\left[\omega_{1}, \omega_{2}\right], \phi_{1}, \ldots, \phi_{u}\right)
\end{aligned}
$$




$$
\begin{aligned}
& +\sum_{q \in Q} a_{q} X_{+} \operatorname{div}_{\pi+1} \ldots X_{+} \operatorname{div}_{i_{\alpha+1}} \\
& \quad \times C_{g}^{h, i_{\pi+1} \ldots i_{\alpha+1}}\left(\Omega_{1}, \ldots, \Omega_{p}, \nabla_{+}\left[\omega_{1}, \omega_{2}\right], \phi_{1}, \ldots, \phi_{u}\right) \\
& +\sum_{j \in J} a_{j} C_{g}^{j}\left(\Omega_{1}, \ldots, \Omega_{p},\left[\omega_{1}, \omega_{2}\right], \phi_{1}, \ldots, \phi_{u}\right),
\end{aligned}
$$

where the last two linear combinations on the left-hand side of the equality are generic linear combinations in the form described in the claim of Lemma 2.3. ${ }^{40}$ On the other hand,

$$
\sum_{t \in T_{k}} a_{t} C_{g}^{t, i_{\pi+1} \ldots i_{\alpha+k}}\left(\Omega_{1}, \ldots, \Omega_{p},\left[\omega_{1}, \omega_{2}\right], \phi_{1}, \ldots, \phi_{u}\right)
$$

is a linear combination of acceptable $(\alpha-\pi+k)$-tensor fields in the form (2-36) with a simple character $\vec{\kappa}_{\text {simp }}$, and with two antisymmetric factors $\nabla \omega_{1}, \nabla \omega_{2}$ that do not contain a free index. We then claim that modulo complete contractions of length $\geq \sigma+u+1$ we can write

$$
\begin{aligned}
& \sum_{t \in T_{k}} a_{t} X_{+} \operatorname{div}_{i_{1}} \ldots X_{+} \operatorname{div}_{i_{a+k}} \\
& \quad \times C_{g}^{t, i_{1} \ldots i_{a+k}}\left(\Omega_{1}, \ldots, \Omega_{p},\left[\omega_{1}, \omega_{2}\right], \phi_{1}, \ldots, \phi_{u}\right) \\
& =\sum_{t \in T_{k+1}} a_{t} X_{+} \operatorname{div}_{i_{\pi+1}} \ldots X_{+} \operatorname{div}_{i_{a+k+1}} \\
& \quad \times C_{g}^{t, i_{\pi+1} \ldots i_{a+k+1}}\left(\Omega_{1}, \ldots, \Omega_{p},\left[\omega_{1}, \omega_{2}\right], \phi_{1}, \ldots, \phi_{u}\right) \\
& +\sum_{q \in Q} a_{q} X_{+} \operatorname{div}_{i_{1}} \ldots X_{+} \operatorname{div}_{i_{a+1}} \\
& \times C_{g}^{q, i_{1} \ldots i_{a+1}}\left(\Omega_{1}, \ldots, \Omega_{p}, \nabla_{+}\left[\omega_{1}, \omega_{2}\right], \phi_{1}, \ldots, \phi_{u}\right) \\
& +\sum_{j \in J} a_{j} C_{g}^{j}\left(\Omega_{1}, \ldots, \Omega_{p},\left[\omega_{1}, \omega_{2}\right], \phi_{1}, \ldots, \phi_{u}\right),
\end{aligned}
$$

with the same notational conventions as above.

We observe that if we can show the above two claims, then Lemma 2.3 will follow by iterative repetition of the second claim.

We will now show the four lemmas above.

Proof of Lemmas 2.8 and 2.10. Lemma 2.8 is a direct consequence of Lemma 4.10 in [A 2010]. ${ }^{41}$ Lemma 2.10 can be proven in two steps: First, by Lemma 2.5 we derive that there exists a linear combination of acceptable $(a+k+1)$-tensor fields

\footnotetext{
${ }^{40}$ In Lemma 2.3, $Q$ is called $V$.

${ }^{41}$ Observe that our hypotheses on the tensor fields in the equation in Lemma 2.1 not being "bad" ensure that we do not fall under the "forbidden" cases of Lemma 4.10 in [A 2010].
} 
(indexed in $X$ below) with a $u$-simple character $\vec{\kappa}_{\text {simp }}$ so that

$$
\begin{aligned}
&(2-43) \sum_{t \in T_{k}} a_{t} C_{g}^{t, i_{1} \ldots i_{a+k}}\left(\Omega_{1}, \ldots, \Omega_{p},\left[\omega_{1}, \omega_{2}\right], \phi_{1}, \ldots, \phi_{u}\right) \nabla_{i_{1}} v \ldots \nabla_{i_{a+k}} v \\
&-\sum_{t \in T_{k+1}} a_{t} X_{*} \operatorname{div}_{i_{a+k+1}} C_{g}^{t, i_{\pi+1} \ldots i_{a+k+1}}\left(\Omega_{1}, \ldots, \Omega_{p},\left[\omega_{1}, \omega_{2}\right], \phi_{1}, \ldots, \phi_{u}\right) \\
& \quad \times \nabla_{i_{1}} v \ldots \nabla_{i_{a+k}} v \\
&=\sum_{j \in J} a_{j} C_{g}^{j}\left(\Omega_{1}, \ldots, \Omega_{p},\left[\omega_{1}, \omega_{2}\right], \phi_{1}, \ldots, \phi_{u}, v^{a+k}\right),
\end{aligned}
$$

where the complete contractions indexed in $J$ have length $\sigma+a+k+1$ and are simply subsequent to $\vec{\kappa}_{\text {simp. }}$. Then, making each factor $\nabla v$ in the above into an $X_{+}$div, we derive Lemma 2.10.

Proof of Lemma 2.7. We have denoted by $\vec{\kappa}_{\text {simp }}$ the simple character of our tensor fields. We distinguish two cases: In Case A there is a factor $\nabla^{(m)} R_{i j k l}$ in $\vec{\kappa}_{\text {simp }}$, and in Case B there is no such factor.

We denote $\alpha+1=\gamma$, for brevity.

Now we break the set $H_{2}^{b}$ into subsets: In Case A we say that $h \in H_{2}^{b,+}$ if $\nabla Y$ contracts against an internal index of a factor $\nabla^{(m)} R_{i j k l}$. In Case B we say that $h \in H_{2}^{b,+}$ if $\nabla Y$ contracts against one of the indices ${ }_{k}, l$ in a factor $S_{*} \nabla^{(v)} R_{i j k l}$.

We define $H_{2}^{b,-}=H_{2}^{b} \backslash H_{2}^{b,+}$.

In each of the above cases and subcases we treat the term $\nabla Y$ as a term $\nabla \phi_{u+1}$ in our lemma hypothesis. Then, by applying the first claim in Lemma 4.10 in [A $2010]^{42}$ to our lemma hypothesis and then making each $\nabla v$ into an $X_{*}$ div, we derive that we can write

$$
\begin{array}{r}
\text { (2-44) } \quad X_{*} \operatorname{div}_{i_{\pi+1}} \ldots X_{*} \operatorname{div}_{i_{\gamma}} \sum_{h \in H_{2}^{b,+}} a_{h} C_{g}^{h, i_{\pi+1} \ldots i_{\alpha}, i_{\gamma}}\left(\Omega_{1}, \ldots, \Omega_{p}, Y, \phi_{1}, \ldots, \phi_{u}\right) \\
=X_{*} \operatorname{div}_{i_{\pi+1}} \ldots X_{*} \operatorname{div}_{i_{\gamma}} \sum_{h \in H_{2}^{b, *,-}} a_{h} C_{g}^{h, i_{\pi+1} \ldots i_{\alpha}, i_{\gamma}}\left(\Omega_{1}, \ldots, \Omega_{p}, Y, \phi_{1}, \ldots, \phi_{u}\right) \\
+\sum_{j \in J} a_{j} C_{g}^{j}\left(\Omega_{1}, \ldots, \Omega_{p}, Y, \phi_{1}, \ldots, \phi_{u}\right),
\end{array}
$$

where $\sum_{h \in H_{2}^{b, *,-}} a_{h} C_{g}^{h, i_{\pi+1} \ldots i_{\alpha}, i_{\gamma}}\left(\Omega_{1}, \ldots, \Omega_{p}, Y, \phi_{1}, \ldots, \phi_{u}\right)$ stands for a generic linear combination as defined above (i.e., it is in the general form $\sum_{h \in H_{2}^{b}} \ldots$ but the factor $\nabla Y$ is not contracting against a special index in any factor $\nabla^{(m)} R_{i j k l}$

${ }^{42}$ By weight considerations, since we started out with no "bad terms" in Lemma 2.1, we will not encounter no "forbidden tensor fields" for Lemma 4.10 in [A 2010]. 
or $\left.S_{*} \nabla^{(v)} R_{i j k l}\right) .^{43}$ On the other hand, each $C_{g}^{j}\left(\Omega_{1}, \ldots, \Omega_{p}, Y, \phi_{1}, \ldots, \phi_{u}\right)$ is a complete contraction with a simple character that is subsequent to $\vec{\kappa}_{\text {simp }}$.

Thus, by virtue of (2-44), we reduce ourselves to the case where $H_{2}^{b,+}=\varnothing$. We will then show Lemma 2.7 separately in Cases A and B, under the assumption that $H_{2}^{b,+}=\varnothing$.

Proof of Lemma 2.7 in Case A. We will define the $C$-crucial factor, for the purposes of this proof only: We denote by Set the set of numbers $u$ for which $\nabla \phi_{u}$ contracts against one of the factors $\nabla^{(m)} R_{i j k l}$. If Set $\neq \varnothing$, we define $u_{+}$to be the minimum element of Set, and we pick out the factor $\nabla^{(m)} R_{i j k l}$ in each $C^{h}$ against which $\nabla \phi_{u_{+}}$contracts. We call that factor $\nabla^{(m)} R_{i j k l} C$-crucial. If $S e t=\varnothing$, we will say the $C$-crucial factors and will mean any of the factors $\nabla^{(m)} R_{i j k l}$.

Now we pick out the subset $H_{2}^{b, *} \subset H_{2}^{b}$, that is defined by the rule $h \in H_{2}^{b \text {,* }}$ if $\nabla Y$ contracts against the (one of the) $C$-crucial factor.

Now, for each $h \in H_{2}^{a}$ we denote by

$$
\operatorname{Hit}_{\operatorname{div}_{i_{\gamma}}} C_{g}^{h, i_{\pi+1} \ldots i_{\alpha+1}}\left(\Omega_{1}, \ldots, \Omega_{p}, Y, \phi_{1}, \ldots, \phi_{u}\right)
$$

the sublinear combination in $X_{*} \operatorname{div}_{i_{\gamma}} C_{g}^{h, i_{\pi+1} \ldots i_{\alpha+1}}\left(\Omega_{1}, \ldots, \Omega_{p}, Y, \phi_{1}, \ldots, \phi_{u}\right)$ that arises when $\nabla_{i_{\gamma}}$ hits the (one of the) $C$-crucial factor. ${ }^{44}$ It then follows that

$$
\begin{aligned}
& \sum_{h \in H_{2}^{a}} a_{h} X_{*} \operatorname{div}_{i_{\pi+1}} \ldots X_{*} \operatorname{div}_{i_{\alpha}} \operatorname{Hit}_{\operatorname{div}_{i_{\gamma}}} C_{g}^{h, i_{\pi+1} \ldots i_{\alpha+1}}\left(\Omega_{1}, \ldots, \Omega_{p}, Y, \phi_{1}, \ldots, \phi_{u}\right) \\
& +\sum_{h \in H_{2}^{b, *}} a_{h} X_{*} \operatorname{div}_{i_{\pi+1}} \ldots X_{*} \operatorname{div}_{i_{\gamma}} C_{g}^{h, i_{\pi+1} \ldots i_{\alpha+1}}\left(\Omega_{1}, \ldots, \Omega_{p}, Y, \phi_{1}, \ldots, \phi_{u}\right) \\
& =\sum_{j \in J} a_{j} C_{g}^{j}\left(\Omega_{1}, \ldots, \Omega_{p}, Y, \phi_{1}, \ldots, \phi_{u}\right),
\end{aligned}
$$

where each $C_{g}^{j}$ has the factor $\nabla Y$ contracting against the $C$-crucial factor $\nabla^{(m)} R_{i j k l}$ and is simply subsequent to $\vec{\kappa}_{\text {simp }}$.

Denote the $(u+1)$-simple character (the one defined by $\nabla \phi_{1}, \ldots, \nabla \phi_{u+1}=\nabla Y$ ) of the tensor fields Hit $\operatorname{div}_{i_{\gamma}} C_{g}^{h, i_{\pi+1} \ldots i_{\alpha}, i_{\gamma}}\left(\Omega_{1}, \ldots, \Omega_{p}, Y, \phi_{1}, \ldots, \phi_{u}\right)$ by $\vec{\kappa}_{\text {simp }}^{\prime}$. (Observe that they all have the same $(u+1)$-simple character.)

${ }^{43}$ Recall that a special index in a factor $\nabla^{(m)} R_{i j k l}$ is an internal index, while a special index in a factor $S_{*} \nabla^{(v)} R_{i j k l}$ is an index ${ }_{k}, l$.

${ }^{44}$ Recall that $i_{\gamma}$ is the free index that belongs to $\nabla Y$. 
We observe that by applying Corollary 1 in [A 2010] to (2-45) (all tensor fields are acceptable and have the same simple character $\vec{\kappa}_{\text {simp }}^{\prime}$ ), ${ }^{45}$ we obtain

$$
\begin{gathered}
\sum_{h \in H_{2}^{a}} a_{h} \operatorname{Hit}_{\operatorname{div}_{i_{\gamma}} C_{g}^{h, i_{\pi+1} \ldots i_{\alpha}}\left(\Omega_{1}, \ldots, \Omega_{p}, Y, \phi_{1}, \ldots, \phi_{u}\right) \nabla_{i_{\pi+1}} v \ldots \nabla_{i_{\alpha}} v} \\
+\sum_{u \in U} a_{u} X \operatorname{div}_{i_{\alpha+1}} C_{g}^{u, i_{\pi+1} \ldots i_{\alpha}, i_{\alpha+1}}\left(\Omega_{1}, \ldots, \Omega_{p}, Y, \phi_{1}, \ldots, \phi_{u}\right) \\
\quad \times \nabla_{i_{\pi+1}} v \ldots \nabla_{i_{\alpha}} v \\
=\sum_{j \in J} a_{j} C_{g}^{j, i_{\pi+1} \ldots i_{\alpha}}\left(\Omega_{1}, \ldots, \Omega_{p}, Y, \phi_{1}, \ldots, \phi_{u}\right) \nabla_{i_{\pi+1}} v \ldots \nabla_{i_{\alpha}} v=0,
\end{gathered}
$$

where the tensor fields indexed in $U$ are acceptable (we are treating $\nabla Y$ as a factor $\nabla \phi_{u+1}$ ), have a simple character $\vec{\kappa}_{\text {simp }}^{\prime}$ and each $C^{j}$ is simply subsequent to $\vec{\kappa}_{\text {simp }}^{\prime}$.

But then, our first claim follows almost immediately. We recall the operation Erase $_{\nabla Y}[\ldots]$ from the Appendix in [A 2012] which acts on the complete contractions in the above by erasing the factor $\nabla Y$ and the (derivative) index that it contracts against. Then, since (2-46) holds formally, we have that the tensor field required for Lemma 2.7 is

$$
\sum_{u \in U} a_{u} \operatorname{Erase}_{\nabla Y}\left[C_{g}^{u, i_{\pi+1} \ldots i_{\alpha}, i_{\alpha+1}}\left(\Omega_{1}, \ldots, \Omega_{p}, Y, \phi_{1}, \ldots, \phi_{u}\right)\right] \cdot \nabla_{i_{\gamma}} Y .
$$

Proof of Lemma 2.7 in Case B. We again distinguish two subcases: In Subcase (i) there is some nonsimple factor $S_{*} \nabla^{(v)} R_{i j k l}$ in $\vec{\kappa}_{\text {simp }}$ or a nonsimple factor $\nabla^{(B)} \Omega_{x}$ contracting against two factors $\nabla \phi_{h}^{\prime}$ in $\vec{\kappa}_{\text {simp. In Subcase (ii) there are no such }}$ factors.

In Subcase (i), we arbitrarily pick out one factor $S_{*} \nabla^{(v)} R_{i j k l}$ or $\nabla^{(B)} \Omega_{x}$ with the properties described above and call it the $D$-crucial factor. In this first subcase we will show our claim for the whole sublinear combination $\sum_{h \in H_{2}^{a}} \ldots$ in one piece.

In Subcase (ii), we will introduce some notation: We will examine each factor $T=S_{*} \nabla^{(v)} R_{i j k l}, T=\nabla^{(B)} \Omega_{x}$ in each tensor field $C_{g}^{h, i_{\pi+1} \ldots i_{\alpha}, i_{\alpha+1}}$ and define its "measure" as follows: If $T=S_{*} \nabla^{(v)} R_{i j k l}$ then its "measure" will stand for its total number of free indices plus $\frac{1}{2}$. If $T=\nabla^{(B)} \Omega_{x}$ then its "measure" will stand for its total number of free indices plus the number of factors $\nabla \phi_{h}$ against which it contracts.

We divide the index set $H_{2}^{a}$ into subsets according to the measure of any given factor. We denote by $M$ the maximum measure among all factors among the tensor fields $C_{g}^{h, i_{\pi+1} \ldots i_{\alpha}, i_{\alpha+1}}, h \in H_{2}^{a}$. We denote by $H_{a}^{2, *} \subset H_{2}^{a}$ the index set of the tensor fields that contain a factor of maximum measure. We will show the claim of

${ }^{45}$ Notice that by weight considerations, since we started out with no "bad" terms in the hypothesis of Lemma 2.1, there is no danger of falling under a "forbidden case" of that corollary. 
Lemma 2.7 for the sublinear combination $\sum_{h \in H_{a}^{2, *}} \ldots$ Clearly, if we can do this, then Lemma 2.7 will follow by induction.

We will prove Lemma 2.7 in the second subcase (which is the hardest). The proof in the first subcase follows by the same argument, only by disregarding any reference to $M$ free indices belonging to a given factor and so on.

Proof of Lemma 2.7 in Case B for the sublinear combination $\sum_{h \in H_{a}^{2, *}} \ldots$ We will further divide $H_{a}^{2, *}$ into subsets, $H_{a}^{2, *, k}, k=1, \ldots, \sigma$, according to the factor of maximum measure: First, we order the factors $S_{*} \nabla^{(v)} R_{i j k l}, \ldots \nabla^{(p)} \Omega_{h}$ in $\vec{\kappa}_{\text {simp }}$, and label them $T_{1}, \ldots, T_{\sigma}$ (observe each factor is well-defined in $\vec{\kappa}_{\text {simp }}$, because we are in Case B). We then say that $h \in H_{2}^{a, *, 1}$ if in $C_{g}^{u, i_{\pi+1} \ldots i_{\alpha}}$ the factor $T_{1}$ has measure $M$. We say that $h \in H_{2}^{a, *, 2}$ if in $C_{g}^{u, i_{\pi+1} \ldots i_{\alpha}}$ the factor $T_{2}$ has measure $M$ and $T_{1}$ has measure less than $M$, and so on. We will then prove our claim for each of the index sets $h \in H_{2}^{a, *, k}:{ }^{46}$ We arbitrarily pick a $k \leq K$ and show our claim for $\sum_{h \in H_{a}^{2, *, k}} \ldots$

For the purposes of this proof, we call the factor $T_{k}$ the $D$-crucial factor.

Now we pick out the subset $H_{2}^{b, k} \subset H_{2}^{b}$, that is defined by the rule $h \in H_{2}^{b, k}$ if and only if $\nabla Y$ is contracting against the $D$-crucial factor $T_{k}$.

Now, for each $h \in H_{2}^{a}$ we denote by

$$
\text { Hit } \operatorname{div}_{i_{\gamma}} C_{g}^{h, i_{\pi+1} \ldots i_{\alpha+1}}\left(\Omega_{1}, \ldots, \Omega_{p}, Y, \phi_{1}, \ldots, \phi_{u}\right)
$$

the sublinear combination in $X \operatorname{div}_{i_{\gamma}} C_{g}^{h, i_{\pi+1} \ldots i_{\alpha+1}}\left(\Omega_{1}, \ldots, \Omega_{p}, Y, \phi_{1}, \ldots, \phi_{u}\right)$ that arises when $\nabla_{i_{\gamma}}$ hits the $D$-crucial factor. ${ }^{47}$ It then follows that

$$
\begin{aligned}
& \sum_{h \in H_{2}^{a}} a_{h} X \operatorname{div}_{i_{\pi+1}} \ldots X \operatorname{div}_{i_{\alpha}} \operatorname{Hit}_{\operatorname{div}_{i_{\gamma}} C_{g}^{h, i_{\pi+1} \ldots i_{\alpha+1}}\left(\Omega_{1}, \ldots, \Omega_{p}, Y, \phi_{1}, \ldots, \phi_{u}\right)} \\
& \quad+\sum_{h \in H_{2}^{b, k}} a_{h} X \operatorname{div}_{i_{\pi+1}} \ldots X \operatorname{div}_{i_{\gamma}} C_{g}^{h, i_{\pi+1} \ldots i_{\alpha+1}}\left(\Omega_{1}, \ldots, \Omega_{p}, Y, \phi_{1}, \ldots, \phi_{u}\right) \\
& =\sum_{j \in J} a_{j} C_{g}^{j}\left(\Omega_{1}, \ldots, \Omega_{p}, Y, \phi_{1}, \ldots, \phi_{u}\right)
\end{aligned}
$$

where each $C_{g}^{j}$ has the factor $\nabla Y$ contracting against the $D$-crucial factor and is simply subsequent to $\vec{\kappa}_{\text {simp }}$.

Denote the $(u+1)$-simple character (the one defined by $\nabla \phi_{1}, \ldots, \nabla \phi_{u+1}=\nabla Y$ ) of the tensor fields $\operatorname{Hit}_{\operatorname{div}_{i_{\gamma}}} C_{g}^{h, i_{\pi+1} \ldots i_{\alpha}, i_{\gamma}}\left(\Omega_{1}, \ldots, \Omega_{p}, Y, \phi_{1}, \ldots, \phi_{u}\right)$ by $\vec{\kappa}_{\text {simp }}^{\prime}$. (Observe that they all have the same $(u+1)$-simple character.)

We apply Corollary 1 in [A 2010] to (2-47) (all tensor fields are acceptable and have the same simple character $\vec{\kappa}_{\text {simp }}^{\prime}$ ) and then pick out the sublinear combination

${ }^{46}$ Again we observe that if we can prove this then Lemma 2.7 in Case B will follow by induction.

${ }^{47}$ Recall that $i_{\gamma}=i_{\alpha+1}$ belongs to $\nabla Y$ by hypothesis. 
where there are $M$ factors $\nabla v$ or $\nabla \phi_{h}$ or $\nabla \phi_{h}^{\prime}$ contracting against $T_{k}$, obtaining

$$
\begin{gathered}
\sum_{h \in H_{2}^{a, *, k}} a_{h} \operatorname{Hit}_{\operatorname{div}_{i_{\gamma}} C_{g}, i_{\pi+1} \ldots i_{\alpha+1}}\left(\Omega_{1}, \ldots, \Omega_{p}, Y, \phi_{1}, \ldots, \phi_{u}\right) \nabla_{i_{\pi+1}} v \ldots \nabla_{i_{\alpha}} v \\
+\sum_{u \in U} a_{u} X \operatorname{div}_{i_{\alpha+1}} C_{g}^{h, i_{\pi+1} \ldots i_{\alpha}, i_{\alpha+1}}\left(\Omega_{1}, \ldots, \Omega_{p}, Y, \phi_{1}, \ldots, \phi_{u}\right) \\
\quad \times \nabla_{i_{\pi+1}} v \ldots \nabla_{i_{\alpha}} v \\
=\sum_{j \in J} a_{j} C_{g}^{j, i_{\pi+1} \ldots i_{\alpha}}\left(\Omega_{1}, \ldots, \Omega_{p}, Y, \phi_{1}, \ldots, \phi_{u}\right) \nabla_{i_{\pi+1}} v \ldots \nabla_{i_{\alpha}} v=0,
\end{gathered}
$$

where the tensor fields indexed in $U$ are acceptable and have a simple character $\vec{\kappa}_{\text {simp }}^{\prime}$ and each $C^{j}$ is simply subsequent to $\vec{\kappa}_{\text {simp }}^{\prime}$.

Now, observe that if $M \geq \frac{3}{2}$, we can apply the eraser to $\nabla Y$ (see the Appendix in [A 2012]) and the index it contracts against in the $D$-crucial factor and derive our conclusion as in Case A.

On the other hand, in the remaining cases ${ }^{48}$ the above argument cannot be directly applied. In those cases, we derive our claim as follows:

In the case $M=1$ the $D$-crucial factor is of the form $\nabla^{(p)} \Omega_{h}$, then we cannot directly derive our claim by the above argument, because if for some tensor fields in $U$ above we have $\nabla Y$ contracting according to the pattern $\nabla_{i} Y \nabla^{i j} \Omega_{h} \nabla_{j} \psi$ (where $\psi=v$ or $\psi=\phi_{h}$ ), then we will not obtain acceptable tensor fields after we apply the eraser. Therefore, if $M=1$ and the $D$-crucial factor is of the form $\nabla^{(p)} \Omega_{h}$, we apply Lemma 4.6 in [A 2010] to (2-48) (treating the factors $\nabla v$ as factors $\nabla \phi)^{49}$ to obtain a new equation in the form (2-48), where for any tensor field indexed in $U$ the factor $\nabla Y$ contracts against a factor $\nabla^{(l)} \Omega_{h}, l \geq 3 .{ }^{50}$ Then, applying the eraser as explained, we derive our Lemma 2.7 in this case.

When $M=\frac{1}{2}$ or $M=0$, then we first apply the inductive assumptions of Corollaries 3 and 2 in [A 2010] (respectively) to (2-48), ${ }^{51}$ in order to assume with no loss of generality that for each tensor field indexed in $U$ there, the factor $\nabla Y$ either contracts against a factor $\nabla^{(B)} \Omega_{h}, B \geq 3$ or a factor $S_{*} \nabla^{(v)} R_{i j k l}, v \geq 1$. Then the eraser can be applied and it produces acceptable tensor fields. Hence, applying Erase $_{\nabla Y}$ to (2-48) we derive our claim.

${ }^{48}$ Observe that the remaining cases are when $M=0, M=\frac{1}{2}, M=1$.

${ }^{49}$ Furthermore, we can observe that we do not fall under a "forbidden case" of Lemma 4.1 in [A 2010], by weight considerations, and since the tensor fields in our lemma assumption are not "bad".

${ }^{50}$ Note that the weight becomes less negative, hence Lemma 4.10 in [A 2010] applies.

${ }^{51} \mathrm{By}$ our assumptions there will be a removable index in these cases. Hence our extra requirements of those lemmas are fulfilled. 
Proof of Lemma 2.9. We rewrite the hypothesis of Lemma 2.3 (which is also the hypothesis of Lemma 2.9) as

$$
\begin{gathered}
\sum_{h \in H_{2}} a_{h} X_{*} \operatorname{div}_{i_{\pi+1}} \ldots X_{*} \operatorname{div}_{i_{\alpha+1}}\left\{C_{g}^{h, i_{1} \ldots i_{\alpha+1}}\left(\Omega_{1}, \ldots, \Omega_{p}, \omega_{1}, \omega_{2}, \phi_{1}, \ldots, \phi_{u}\right)\right. \\
\left.\quad-\operatorname{Switch}[C]_{g}^{h, i_{\pi+1} \ldots i_{\alpha+1}}\left(\Omega_{1}, \ldots, \Omega_{p}, \omega_{1}, \omega_{2}, \phi_{1}, \ldots, \phi_{u}\right)\right\} \\
=\sum_{j \in J} a_{j} C_{g}^{j}\left(\Omega_{1}, \ldots, \Omega_{p},\left[\omega_{1}, \omega_{2}\right], \phi_{1}, \ldots, \phi_{u}\right) .
\end{gathered}
$$

Here the operation Switch interchanges the indices $a$ and $b$ in the two factors $\nabla_{a} \omega_{1}$, $\nabla_{b} \omega_{2}$.

Notational conventions: We have again denoted by $H_{2}^{a} \subset H_{2}$ the index set of those vector fields for which one of the free indices (say $i_{\alpha+1}$ ) belongs to a factor $\nabla \omega_{1}$ or $\nabla \omega_{2}$. With no loss of generality we assume that for each $h \in H_{2}^{a}$, the index $i_{\alpha+1}$ belongs to the factor $\nabla \omega_{1}$. We can clearly do this, due to the antisymmetry of the factors $\nabla \omega_{1}, \nabla \omega_{2}$.

We have defined $H_{2}^{b}=H_{2} \backslash H_{2}^{a}$. For each $h \in H_{2}^{b}$ we denote by $T_{\omega_{1}}, T_{\omega_{2}}$ the factors against which $\nabla \omega_{1}, \nabla \omega_{2}$ contract. Also, for each $h \in H_{2}^{a}$ we will denote by $T_{\omega_{2}}$ the factor against which $\nabla \omega_{2}$ contracts. ${ }^{52}$

For each $h \in H_{2}$, we will call the factors $T_{\omega_{1}}, T_{\omega_{2}}$ against which $\nabla \omega_{1}$ or $\nabla \omega_{2}$ are contracting "problematic" in the following cases: If $T_{\omega_{1}}$ or $T_{\omega_{2}}$ is of the form $\nabla^{(m)} R_{i j k l}$ and $\nabla \omega_{1}$ or $\nabla \omega_{2}$ contracts against an internal index; or if $T_{\omega_{1}}$ or $T_{\omega_{2}}$ is of the form $S_{*} \nabla^{(v)} R_{i j k l}$ and the factor $\nabla \omega_{1}$ or $\nabla \omega_{2}$ contracts against one of the indices $k$ or $l$.

We then define a few subsets of $H_{2}^{a}, H_{2}^{b}$ :

Definition. We define $H_{2, * *}^{b}$ to be the index set of the tensor fields $C_{g}^{h, i_{\pi+1} \ldots i_{\alpha+1}}$ for which $\nabla \omega_{1}, \nabla \omega_{2}$ contract against different factors and both $T_{\omega_{1}}$ and $T_{\omega_{2}}$ are problematic.

We define $H_{2, *}^{a} \subset H_{2}^{a}$ to be the index set of the tensor fields $C_{g}^{h, i_{\pi+1} \ldots i_{\alpha+1}} \mathrm{~s}$ for which $T_{\omega_{2}}$ is problematic.

We define $H_{2, *}^{b}$ to stand for the index set of the tensor fields $C_{g}^{h, i_{\pi+1} \ldots i_{\alpha+1}} \mathrm{~s}$ for which either $T_{\omega_{1}}=T_{\omega_{2}}$ or $T_{\omega_{1}} \neq T_{\omega_{2}}$ and one of the factors $T_{\omega_{1}}, T_{\omega_{2}}$ is problematic.

Abusing notation, we will use the symbols $\sum_{h \in H_{2, *}^{b}}$ and so on to denote generic linear combinations as above, when these symbols appear in the right-hand sides of the equations below.

${ }^{52}$ Note that the definition of $T_{\omega_{1}}, T_{\omega_{2}}$ depends on $h$; however, to simplify notation we suppress the index $h$ that should appear in $T_{\omega_{1}}, T_{\omega_{2}}$. 
We then state three preparatory claims. First, we claim that we can write

$$
\begin{aligned}
& \sum_{h \in H_{2, * *}^{b}} a_{h} X_{+} \operatorname{div}_{i_{\pi+1}} \ldots X_{+} \operatorname{div}_{i_{\alpha+1}} \\
& \times\left\{C_{g}^{h, i_{\pi+1} \ldots i_{\alpha+1}}\left(\Omega_{1}, \ldots, \Omega_{p}, \omega_{1}, \omega_{2}, \phi_{1}, \ldots, \phi_{u}\right)\right. \\
& \left.-\operatorname{Switch}[C]_{g}^{h, i_{\pi+1} \ldots i_{\alpha+1}}\left(\Omega_{1}, \ldots, \Omega_{p}, \omega_{1}, \omega_{2}, \phi_{1}, \ldots, \phi_{u}\right)\right\} \\
& =\sum_{h \in H_{2, *}^{b}} a_{h} X_{+} \operatorname{div}_{i_{\pi+1}} \ldots X_{+} \operatorname{div}_{i_{\alpha+1}} \\
& \times\left\{C_{g}^{h, i_{\pi+1} \ldots i_{\alpha+1}}\left(\Omega_{1}, \ldots, \Omega_{p}, \omega_{1}, \omega_{2}, \phi_{1}, \ldots, \phi_{u}\right)\right. \\
& \left.-\operatorname{Switch}[C]_{g}^{h, i_{\pi+1} \ldots i_{\alpha+1}}\left(\Omega_{1}, \ldots, \Omega_{p}, \omega_{1}, \omega_{2}, \phi_{1}, \ldots, \phi_{u}\right)\right\} \\
& +\sum_{j \in J} a_{j} C_{g}^{j}\left(\Omega_{1}, \ldots, \Omega_{p},\left[\omega_{1}, \omega_{2}\right], \phi_{1}, \ldots, \phi_{u}\right),
\end{aligned}
$$

where the linear combination $\sum_{h \in H_{2, *}^{b}} \ldots$ on the right-hand side stands for a generic linear combination in the form described above. Observe that if we can show (2-50) then we may assume with no loss of generality that $H_{2, * *}^{b}=\varnothing$ in our lemma hypothesis.

Then, assuming that $H_{2, * *}^{b}=\varnothing$ in our lemma hypothesis we will show that there exists a linear combination of $(\alpha-\pi+1)$-tensor fields (indexed in $X$ below) which are in the form (2-5) with a simple character $\vec{\kappa}_{\text {simp }}$ so that

$$
\begin{aligned}
& \sum_{h \in H_{2, *}^{a}} a_{h}\left\{C_{g}^{h, i_{\pi+1} \ldots i_{\alpha+1}}\left(\Omega_{1}, \ldots, \Omega_{p}, \omega_{1}, \omega_{2}, \phi_{1}, \ldots, \phi_{u}\right)\right. \\
& \left.-\operatorname{Switch}[C]_{g}^{h, i_{\pi+1} \ldots i_{\alpha+1}}\left(\Omega_{1}, \ldots, \Omega_{p}, \omega_{1}, \omega_{2}, \phi_{1}, \ldots, \phi_{u}\right)\right\} \nabla_{i_{\pi+1}} v \ldots \nabla_{i_{\alpha+1}} v \\
& -X_{*} \operatorname{div}_{i_{\alpha+2}} \sum_{x \in X} a_{x}\left\{C_{g}^{x, i_{1} \ldots i_{\alpha+1} i_{\alpha+2}}\left(\Omega_{1}, \ldots, \Omega_{p}, \omega_{1}, \omega_{2}, \phi_{1}, \ldots, \phi_{u}\right)\right. \\
& \left.-\operatorname{Switch}[C]_{g}^{h, i_{\pi+1} \ldots i_{\alpha+1}}\left(\Omega_{1}, \ldots, \Omega_{p}, \omega_{1}, \omega_{2}, \phi_{1}, \ldots, \phi_{u}\right)\right\} \nabla_{i_{\pi+1}} v \ldots \nabla_{i_{\alpha+1}} v \\
& +\sum_{h \in H_{2, *}^{b}} a_{h}\left\{C_{g}^{h, i_{1} \ldots i_{\alpha+1}}\left(\Omega_{1}, \ldots, \Omega_{p}, \omega_{1}, \omega_{2}, \phi_{1}, \ldots, \phi_{u}\right)\right. \\
& \left.-\operatorname{Switch}[C]_{g}^{h, i_{\pi+1} \ldots i_{\alpha+1}}\left(\Omega_{1}, \ldots, \Omega_{p}, \omega_{1}, \omega_{2}, \phi_{1}, \ldots, \phi_{u}\right)\right\} \nabla_{i_{\pi+1}} v \ldots \nabla_{i_{\alpha+1}} v \\
& =\sum_{j \in J} a_{j} C_{g}^{j}\left(\Omega_{1}, \ldots, \Omega_{p},\left[\omega_{1}, \omega_{2}\right], \phi_{1}, \ldots, \phi_{u}, v^{\alpha-\pi}\right) .
\end{aligned}
$$

We observe that if we can show the above, we may then assume that $H_{2, *}^{a}=\varnothing$ (and $H_{2, * *}^{b}=\varnothing$ ) in the hypothesis of Lemma 2.9. 
Finally, under the assumption that $H_{2, * *}^{b}=H_{2, *}^{a}=\varnothing$ in our lemma hypothesis, we will show that we can write

$$
\begin{aligned}
& \sum_{h \in H_{2, *}^{b}} a_{h} X_{+} \operatorname{div}_{i_{\pi+1}} \ldots X_{+} \operatorname{div}_{i_{\alpha+1}} \\
& \times\left\{C_{g}^{h, i_{\pi+1} \ldots i_{\alpha+1}}\left(\Omega_{1}, \ldots, \Omega_{p}, \omega_{1}, \omega_{2}, \phi_{1}, \ldots, \phi_{u}\right)\right. \\
& \left.\quad-\quad \operatorname{Switch}[C]_{g}^{h, i_{\pi+1} \ldots i_{\alpha+1}}\left(\Omega_{1}, \ldots, \Omega_{p}, \omega_{1}, \omega_{2}, \phi_{1}, \ldots, \phi_{u}\right)\right\} \\
& =\sum_{h \in H_{2, O K}^{b}} a_{h} X_{+} \operatorname{div}_{i_{\pi+1}} \ldots X_{+} \operatorname{div}_{i_{\alpha+1}} \\
& \quad \times\left\{C_{g}^{h, i_{\pi+1} \ldots i_{\alpha+1}}\left(\Omega_{1}, \ldots, \Omega_{p}, \omega_{1}, \omega_{2}, \phi_{1}, \ldots, \phi_{u}\right)\right. \\
& \left.\quad-\operatorname{Switch}[C]_{g}^{h, i_{\pi+1} \ldots i_{\alpha+1}}\left(\Omega_{1}, \ldots, \Omega_{p}, \omega_{1}, \omega_{2}, \phi_{1}, \ldots, \phi_{u}\right)\right\} \\
& +\sum_{j \in J} a_{j} C_{g}^{j}\left(\Omega_{1}, \ldots, \Omega_{p},\left[\omega_{1}, \omega_{2}\right], \phi_{1}, \ldots, \phi_{u}\right),
\end{aligned}
$$

where the sublinear combination $\sum_{h \in H_{2, O K}^{b}} \ldots$ on the right-hand side stands for a generic linear combination of acceptable tensor fields in the form (2-5) with simple character $\vec{\kappa}_{\text {simp }}$, with no free indices in the factors $\nabla \omega_{1}, \nabla \omega_{2}$ and where the factors $T_{\omega_{1}}, T_{\omega_{2}}$ are not problematic. Therefore, if we can show the above equations, we are reduced to showing Lemma 2.9 under the assumptions that $H_{a, *}^{2}=H_{b, * *}^{2}=$ $H_{b, *}^{2}=\varnothing$.

Sketch of the proof of (2-50), (2-51), (2-52). Equation (2-50) follows by reiterating the proof of the first claim of Lemma 4.10 in [A 2010]. ${ }^{53}$ (2-51) follows by reiterating the proof of the first claim of Lemma 4.10 in [A 2010], but rather than applying Corollary 1 [A 2010] in that proof, we now apply Lemma 2.7 (which we have shown). ${ }^{54}$ Finally, the claim of (2-52) for the sublinear combination in $H_{2, *}^{b}$ where $T_{\omega_{1}} \neq T_{\omega_{2}}$ follows by applying Lemma 2.5..$^{55}$ We can then show that the remaining sublinear combination in $\sum_{h \in H_{2, *}^{b}} \ldots$ must vanish separately (modulo a linear combination $\sum_{j \in J} \ldots$ ) by picking out the sublinear combination in the hypothesis of Lemma 2.10 where both factors $\nabla \omega_{1}, \nabla \omega_{2}$ are contracting against the same factor.

Now, under these additional assumptions that $H_{a, *}^{2}=H_{b, * *}^{2}=H_{b, *}^{2}=\varnothing$, we will show our claim by distinguishing two cases: In Case A there is a factor $\nabla^{(m)} R_{i j k l}$

${ }^{53} \mathrm{By}$ the additional restrictions imposed on the assumption of Lemma 2.3 there is no danger of falling under a "forbidden case" of Corollary 1 in [A 2010].

${ }^{54}$ Observe that the assumption that Lemma 2.3 does not include "forbidden cases" ensures that we will not need to apply Lemma 2.7 in a "forbidden case".

${ }^{55}$ In this case there will be a factor $\nabla \omega_{1}$ or $\nabla \omega_{2}$ contracting against a nonspecial index; therefore there is no danger of falling under a "forbidden" case of Lemma 2.7. 
in $\vec{\kappa}_{\text {simp }}$; in Case B there is no such factor. An important note: We may now use Lemma 2.7, which we have proven earlier in this section.

Proof of Lemma 2.9 in Case A. We define the (set of) $C$-crucial factors (which will necessarily be of the form $\nabla^{(m)} R_{i j k l}$ ) as in the setting of Lemma 2.7. First we prove a mini-claim which only applies to the case where the $C$-crucial factor is unique.

Mini-claim, when the $C$-crucial factor is unique. We then consider the tensor fields $C_{g}^{h, i_{\pi+1} \ldots i_{\alpha+1}}, h \in H_{2}^{a}$ for which $\nabla \omega_{2}$ contracts against the $C$-crucial factor. Notice that by our hypothesis that $H_{a, *}^{2}=\varnothing$, it follows that $\nabla \omega_{2}$ contracts against a derivative index in the $C$-crucial factor. Denote by $H_{2}^{a,+} \subset H_{2}^{a}$ the index set of these tensor fields.

We observe that for each $h \in H_{2}^{a,+}$ we can now construct a tensor field by erasing the index in the factor $\nabla^{(m)} R_{i j k l}$ that contracts against the factor $\nabla \omega_{2}$ and making the index in $\nabla \omega_{2}$ into a free index $i_{\beta}$. We denote this tensor field by $C_{g}^{h, i_{\pi+1} \ldots i_{\alpha+1} i_{\beta}}\left(\Omega_{1}, \ldots, \Omega_{p}, \omega_{1}, \omega_{2}, \phi_{1}, \ldots, \phi_{u}\right)$. By the analogous operation we obtain a tensor field $\operatorname{Switch}\left[C_{g}^{h, i_{\pi+1} \ldots i_{\alpha+1} i_{\beta}}\left(\Omega_{1}, \ldots, \Omega_{p}, \omega_{1}, \omega_{2}, \phi_{1}, \ldots, \phi_{u}\right)\right]$.

It follows that in the case where the $C$-crucial factor is unique, for each $h \in H_{2}^{a,+}$,

$$
\begin{gathered}
X_{*} \operatorname{div}_{i_{\pi+1}} \ldots X_{*} \operatorname{div}_{i_{\alpha+1}}\left\{C_{g}^{h, i_{\pi+1} \ldots i_{\alpha+1}}\left(\Omega_{1}, \ldots, \Omega_{p}, \omega_{1}, \omega_{2}, \phi_{1}, \ldots, \phi_{u}\right)\right. \\
\left.\quad-\quad \operatorname{Switch}[C]_{g}^{h, i_{\pi+1} \ldots i_{\alpha+1}}\left(\Omega_{1}, \ldots, \Omega_{p}, \omega_{1}, \omega_{2}, \phi_{1}, \ldots, \phi_{u}\right)\right\} \\
=X_{*} \operatorname{div}_{i_{\pi+1}} \ldots X_{*} \operatorname{div}_{i_{\alpha+1}} X_{*} \operatorname{div}_{i_{\beta}} \\
\times\left\{C_{g}^{h, i_{\pi+1} \ldots i_{\alpha+1} i_{\beta}}\left(\Omega_{1}, \ldots, \Omega_{p}, \omega_{1}, \omega_{2}, \phi_{1}, \ldots, \phi_{u}\right)\right. \\
\left.\quad-\operatorname{Switch}[C]_{g}^{h, i_{\pi+1} \ldots i_{\alpha+1} i_{\beta}}\left(\Omega_{1}, \ldots, \Omega_{p}, \omega_{1}, \omega_{2}, \phi_{1}, \ldots, \phi_{u}\right)\right\} \\
+\sum_{r \in R} a_{r} X_{*} \operatorname{div}_{i_{\pi+1}} \ldots X_{*} \operatorname{div}_{i_{\alpha+1}} \\
\times\left\{C_{g}^{r, i_{\pi+1} \ldots i_{\alpha+1}}\left(\Omega_{1}, \ldots, \Omega_{p}, \omega_{1}, \omega_{2}, \phi_{1}, \ldots, \phi_{u}\right)\right. \\
\left.\quad-\operatorname{Switch}[C]^{r, i_{\pi+1} \ldots i_{\alpha+1}}\left(\Omega_{1}, \ldots, \Omega_{p}, \omega_{1}, \omega_{2}, \phi_{1}, \ldots, \phi_{u}\right)\right\} \\
+\sum_{j \in J} a_{j} C_{g}^{j}\left(\Omega_{1}, \ldots, \Omega_{p}, \omega_{1}, \omega_{2}, \phi_{1}, \ldots, \phi_{u}\right),
\end{gathered}
$$

where each tensor field $C_{g}^{r, i_{\pi+1} \ldots i_{\alpha+1}}\left(\Omega_{1}, \ldots, \Omega_{p}, \omega_{1}, \omega_{2}, \phi_{1}, \ldots, \phi_{u}\right)$ has the factor $\nabla \omega_{2}$ contracting against some factor other than the $C$-crucial factor.

But we observe that

$$
\begin{aligned}
X_{*} & \operatorname{div}_{i_{\pi+1}} \ldots X_{*} \operatorname{div}_{i_{\alpha+1}} X_{*} \operatorname{div}_{i_{\beta}} \\
\times & \left\{C_{g}^{h, i_{\pi+1} \ldots i_{\alpha+1} i_{\beta}}\left(\Omega_{1}, \ldots, \Omega_{p}, \omega_{1}, \omega_{2}, \phi_{1}, \ldots, \phi_{u}\right)\right. \\
& \left.\quad-\operatorname{Switch}[C]_{g}^{h, i_{\pi+1} \ldots i_{\alpha+1} i_{\beta}}\left(\Omega_{1}, \ldots, \Omega_{p}, \omega_{1}, \omega_{2}, \phi_{1}, \ldots, \phi_{u}\right)\right\}=0 .
\end{aligned}
$$


Therefore, in the case $\operatorname{Set} \neq \varnothing$ or $\operatorname{Set}=\varnothing$ and $\sigma_{1}=1$, we have now reduced Lemma 2.9 to the case where $H_{2}^{a,+}=\varnothing$.

Now (under the assumption that $H_{2}^{a,+}=\varnothing$ when the $C$-crucial factor is unique) we consider the sublinear combination Special in the hypothesis of Lemma 2.9 that consists of complete contractions with $\nabla \omega_{1}$ contracting against the $C$-crucial factor while the factor $\nabla \omega_{2}$ is contracting against some other factor. (If Set $=\varnothing$ and $\sigma_{1}>1$ Special stands for the sublinear combination where $\nabla \omega_{1}$ is contracting against a generic $C$-crucial factor and $\nabla \omega_{2}$ is contracting against some other factor.) In particular, for each $h \in H_{2}^{a}$, since $H_{2}^{a,+}=\varnothing$ we see that the sublinear combination in

$$
\begin{aligned}
\sum_{h \in H_{2}^{a}} a_{h} X_{*} \operatorname{div}_{i_{\pi+1}} \ldots X_{*} \operatorname{div}_{i_{\alpha+1}} & \times\left\{C_{g}^{h, i_{\pi+1} \ldots i_{\alpha+1}}\left(\Omega_{1}, \ldots, \Omega_{p}, \omega_{1}, \omega_{2}, \phi_{1}, \ldots, \phi_{u}\right)\right. \\
& \left.\quad-\operatorname{Switch}[C]_{g}^{h, i_{\pi+1} \ldots i_{\alpha+1}}\left(\Omega_{1}, \ldots, \Omega_{p}, \omega_{1}, \omega_{2}, \phi_{1}, \ldots, \phi_{u}\right)\right\}
\end{aligned}
$$

that belongs to Special is precisely

$$
\begin{aligned}
& \sum_{h \in H_{2}^{a}} a_{h} X_{*} \operatorname{div}_{i_{\pi+1}} \ldots X_{*} \operatorname{div}_{i_{\alpha}} \\
& \times \operatorname{Hit}_{\operatorname{div}_{i_{\alpha+1}}} C_{g}^{h, i_{\pi+1} \ldots i_{\alpha+1}}\left(\Omega_{1}, \ldots, \Omega_{p}, \omega_{1}, \omega_{2}, \phi_{1}, \ldots, \phi_{u}\right) ;
\end{aligned}
$$

(in the case Set $=\varnothing$ and $\sigma_{1}>1$, Hit $\operatorname{div}_{i_{\alpha+1}}$ just means that $\nabla_{i_{\gamma}}$ can hit any factor $\nabla^{(m)} R_{i j k l}$ that is not contracting against $\nabla \omega_{2}$; recall that in the other cases it means that it must hit the unique $C$-crucial factor).

We also consider the tensor fields $C^{h, i_{\pi+1} \ldots i_{\alpha+1}}$, Switch $[C]^{h, i_{\pi+1} \ldots i_{\alpha+1}}, h \in H_{2}^{b}$, for which $\nabla \omega_{1}$ contracts against the $C$-crucial factor and $\nabla \omega_{2}$ does not (or, if there are multiple $C$-crucial factors, where $\nabla \omega_{1}, \nabla \omega_{2}$ contract against different $C$ crucial factors). For this proof, we index all those tensor fields in $H_{2}^{b, \Psi}$ and we will denote them by $C_{g}^{h, i_{\pi+1} \ldots i_{\alpha+1}}$.

Thus we derive

$$
\begin{aligned}
& \sum_{h \in H_{2}^{a}} a_{h} X_{*} \operatorname{div}_{i_{\pi+1}} \ldots X_{*} \operatorname{div}_{i_{\alpha}} \operatorname{Hit}_{\operatorname{div}_{i_{\alpha+1}}} \\
& \times C_{g}^{h, i_{\pi+1} \ldots i_{\alpha+1}}\left(\Omega_{1}, \ldots, \Omega_{p}, \omega_{1}, \omega_{2}, \phi_{1}, \ldots, \phi_{u}\right) \\
& +\sum_{h \in H_{2}^{b, \Psi}} a_{h} X_{*} \operatorname{div}_{i_{\pi+1}} \ldots X_{*} \operatorname{div}_{i_{\alpha+1}} \\
& \times C_{g}^{h, i_{\pi+1} \ldots i_{\alpha+1}}\left(\Omega_{1}, \ldots, \Omega_{p}, \omega_{1}, \omega_{2}, \phi_{1}, \ldots, \phi_{u}\right) \\
& =\sum_{j \in J} a_{j} C_{g}^{j}\left(\Omega_{1}, \ldots, \Omega_{p}, \omega_{1}, \omega_{2}, \phi_{1}, \ldots, \phi_{u}\right) .
\end{aligned}
$$

We group up the vector fields on the left-hand side according to their weak $(u+$ 2)- characters $^{56}$ (defined by $\nabla \phi_{1}, \ldots, \nabla \phi_{u}, \nabla \omega_{1}, \nabla \omega_{2}$ ). (Recall that we started off

${ }^{56}$ See [A 2010] for a definition of this notion. 
with complete contractions with the same $u$-simple characters - so the only new information that we are taking into account is what type of factor $\nabla \omega_{2}$ contracts against.) We consider the set of weak simple characters that we have obtained. We denote this set by $\left\{\vec{\kappa}_{1}, \ldots \vec{\kappa}_{B}\right\}$, and we respectively have the index sets $H_{2}^{a, \vec{\kappa}_{f}}$ and $H_{2}^{b, \vec{\kappa}_{f}}$.

We will show our Lemma 2.9 by replacing the index set $H_{2}^{a}$ by any $H_{2}^{a, \vec{\kappa}_{f}}$, $f \leq B$.

It follows that for each $f \leq B$,

$$
\begin{aligned}
& \sum_{h \in H_{2}^{a, \vec{k}_{f}}} a_{h} X_{*} \operatorname{div}_{i_{\pi+1}} \ldots X_{*} \operatorname{div}_{i_{\alpha}} \operatorname{Hit}_{\operatorname{div}_{i_{\alpha+1}}} \\
& \times C_{g}^{h, i_{\pi+1} \ldots i_{\alpha+1}}\left(\Omega_{1}, \ldots, \Omega_{p}, \omega_{1}, \omega_{2}, \phi_{1}, \ldots, \phi_{u}\right) \\
& +\sum_{h \in H_{2}^{b, \vec{k}_{f}}} a_{h} X_{*} \operatorname{div}_{i_{\pi+1}} \ldots X_{*} \operatorname{div}_{i_{\alpha+1}} \\
& \times C_{g}^{h, i_{\pi+1} \ldots i_{\alpha+1}}\left(\Omega_{1}, \ldots, \Omega_{p}, \omega_{1}, \omega_{2}, \phi_{1}, \ldots, \phi_{u}\right) \\
& =\sum_{j \in J} a_{j} C_{g}^{j}\left(\Omega_{1}, \ldots, \Omega_{p}, \omega_{1}, \omega_{2}, \phi_{1}, \ldots, \phi_{u}\right),
\end{aligned}
$$

where the complete contractions $C_{g}^{j}$ have a $u$-simple character that is subsequent

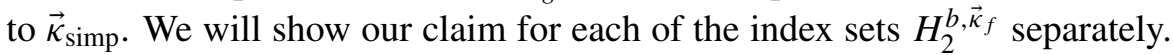

Now, we treat the factors $\nabla \omega_{1}, \nabla \omega_{2}$ in the above as factors $\nabla \phi_{u+1}, \nabla \phi_{u+2}$. We see that since $H_{2, * *}^{b}=H_{b, *}^{2}=H_{a, *}^{2}=\varnothing$, all the tensor fields in the above have the same $(u+2)$-simple character.

Our claim (Lemma 2.9) for the index set $H_{2}^{a, \vec{\kappa}_{f}}$ then follows: First, apply the operator Erase $_{\nabla \omega_{1}}[\ldots]$ to $(2-57) .{ }^{57}$ We are then left with tensor fields (denote them by

$$
\begin{array}{rr}
C_{g}^{h, i_{\pi+1} \ldots i_{\alpha}}\left(\Omega_{1}, \ldots, \Omega_{p}, \omega_{2}, \phi_{1}, \ldots, \phi_{u}\right), & h \in H_{2}^{a, \vec{\kappa}_{f}}, \\
C_{g}^{h, i_{\pi+1} \ldots i_{\alpha+1}}\left(\Omega_{1}, \ldots, \Omega_{p}, \omega_{2}, \phi_{1}, \ldots, \phi_{u}\right), & h \in H_{2}^{b, \vec{\kappa}_{f}},
\end{array}
$$

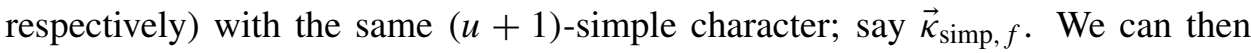
apply Corollary 1 from [A 2010] (since we have weight $-n+2 k, k>0$ by virtue of the eraser - notice that by weight considerations, since we started out with no "bad" tensor fields, there is no danger of falling under a "forbidden case"), to derive that there is a linear combination of acceptable $\alpha$-tensor fields indexed in $V$ below, with $(u+1)$-simple character $\vec{\kappa}_{\text {simp, } f \text {, so that }}$

$$
\sum_{h \in H_{2}^{a, \vec{k}_{f}}} a_{h} C_{g}^{h, i_{\pi+1} \ldots i_{\alpha}}\left(\Omega_{1}, \ldots, \Omega_{p}, \omega_{2}, \phi_{1}, \ldots, \phi_{u}\right) \nabla_{i_{\pi+1}} v \ldots \nabla_{i_{\alpha}} v
$$

${ }^{57}$ See the relevant lemma in the Appendix of [A 2012]. 


$$
\begin{array}{r}
-\sum_{v \in V} a_{v} X_{*} \operatorname{div}_{i_{\alpha+1}} C_{g}^{v, i_{\pi+1} \ldots i_{\alpha+1}}\left(\Omega_{1}, \ldots, \Omega_{p}, \omega_{2}, \phi_{1}, \ldots, \phi_{u}\right) \\
\times \nabla_{i_{\pi+1}} v \ldots \nabla_{i_{\alpha}} v \\
=\sum_{j \in J} a_{j} C_{g}^{j, i_{\pi+1} \ldots i_{\alpha}}\left(\Omega_{1}, \ldots, \Omega_{p}, \omega_{2}, \phi_{1}, \ldots, \phi_{u}\right) \nabla_{i_{\pi+1}} v \ldots \nabla_{i_{\alpha}} v
\end{array}
$$

where each complete contraction indexed in $J$ is $(u+1)$-subsequent to $\vec{\kappa}_{\text {simp, } f}$. In this setting $X_{*} \operatorname{div}_{i}$ just means that in addition to the restrictions imposed on $X \operatorname{div}_{i}$ we are not allowed to hit the factor $\nabla \omega_{2}$.

Then, if we multiply the above equation by an expression $\nabla_{i} \omega_{1} \nabla^{i} v$ and then antisymmetrize the indices ${ }_{a},{ }_{b}$ in the factors $\nabla_{a} \omega_{1}, \nabla_{b} \omega_{2}$ and finally make all $\nabla v \mathrm{~s}$ into $X_{+}$divs, we derive our claim.

Proof of Lemma 2.9 in Case B (when $\sigma_{1}=0$ ). Our proof follows the same pattern as the proof of Lemma 2.7 in Case B.

We again define the "measure" of each factor in each tensor field $C_{g}^{h, i_{\pi+1} \ldots i_{\alpha+1}}$ as in the proof of Case B in Lemma 2.7. Again, let $M$ stand for the maximum measure among all factors in all tensor fields $C_{g}^{h, i_{\pi+1} \ldots i_{\alpha+1}}, h \in H_{2}^{a}$. We denote by $H_{2}^{a, M} \subset H_{2}^{a}$ the index set of the tensor fields for which some factor has measure $M$.

We will further divide $H_{a}^{2, M}$ into subsets $H_{a}^{2, M, k}, k=1, \ldots, \sigma$, according to the factor which has measure $M$ : First, we order the factors $S_{*} \nabla^{(v)} R_{i j k l}, \ldots \nabla^{(p)} \Omega_{h}$ in $\vec{\kappa}_{\text {simp }}$, and label them $T_{1}, \ldots, T_{\sigma}$ (observe each factor is well-defined in $\vec{\kappa}_{\text {simp }}$, because we are in Case B). We then say that $h \in H_{2}^{a, M, 1}$ if in $C_{g}^{h, i_{\pi+1} \ldots i_{\alpha}}, T_{1}$ has measure $M$. We say that $h \in H_{2}^{a, M, 2}$ if in $C_{g}^{h, i_{\pi+1} \ldots i_{\alpha}}, T_{2}$ has measure $M$ and $T_{1}$ has measure less than $M$, and so on. We will then prove our claim for each of the index sets $h \in H_{2}^{a, M, k}$. ${ }^{58}$ We arbitrarily pick a $k \leq \sigma$ and show our claim for $\sum_{h \in H_{a}^{2, M, k}} \ldots$

For the purposes of this proof, we call the factor $T_{k}$ the $D$-crucial factor (in this setting the $D$-crucial factor is unique).

Now, we pick out the subset $H_{2}^{b, k,+} \subset H_{2}^{b}$ that is defined by the rule $h \in H_{2}^{b, k}$ if and only if $\nabla \omega_{1}$ contracts against the $D$-crucial factor $T_{k}$. We also pick out the subset $H_{2}^{b, k,-} \subset H_{2}^{b}$ that is defined by the rule $h \in H_{2}^{b, k}$ if and only if $\nabla \omega_{2}$ contracts against the $D$-crucial factor $T_{k}$. Finally, we define $H_{2}^{a,} \subset H_{2}^{a}, H_{2}^{a,-} \subset H_{2}^{a}$ to stand for the index set of tensor fields for which $\nabla \omega_{2}$ contracts against the $D$-crucial factor.

Now, for each $h \in H_{2}^{a}$ we denote by

$$
\operatorname{Hit}_{\operatorname{div}_{i_{\gamma}}} C_{g}^{h, i_{\pi+1} \ldots i_{\alpha+1}}\left(\Omega_{1}, \ldots, \Omega_{p}, \omega_{1}, \omega_{2}, \phi_{1}, \ldots, \phi_{u}\right)
$$

${ }^{58}$ Again we observe that if we can prove this then Lemma 2.9 in Case B will follow by induction. 
the sublinear combination in $X \operatorname{div}_{i_{\gamma}} C_{g}^{h, i_{\pi+1} \ldots i_{\alpha+1}}\left(\Omega_{1}, \ldots, \Omega_{p}, \omega_{1}, \omega_{2}, \phi_{1}, \ldots, \phi_{u}\right)$ that arises when $\nabla_{i_{\gamma}}$ hits the $D$-crucial factor. It then follows that

$$
\begin{aligned}
& \begin{aligned}
\sum_{h \in H_{2}^{a}} a_{h} X_{*} \operatorname{div}_{i_{\pi+1}} \ldots X_{*} \operatorname{div}_{i_{\alpha}} & \\
& \times \operatorname{Hit}_{\operatorname{div}_{i_{\gamma}}} C_{g}^{h, i_{\pi+1} \ldots i_{\alpha+1}}\left(\Omega_{1}, \ldots, \Omega_{p}, \omega_{1}, \omega_{2}, \phi_{1}, \ldots, \phi_{u}\right)
\end{aligned} \\
& -\sum a_{h} X_{*} \operatorname{div}_{i_{\pi+1}} \ldots X_{*} \operatorname{div}_{i_{\alpha+1}} \\
& h \in H_{2}^{a^{\sim}} \times \operatorname{Switch}[C]_{g}^{h, i_{\pi+1} \ldots i_{\alpha+1}}\left(\Omega_{1}, \ldots, \Omega_{p}, \omega_{1}, \omega_{2}, \phi_{1}, \ldots, \phi_{u}\right) \\
& \begin{aligned}
+\sum_{h \in H_{2}^{b, k,+}} a_{h} X \operatorname{div}_{i_{\pi+1}} \ldots X \operatorname{div}_{i_{\gamma}} \\
\times C_{g}^{h, i_{\pi+1} \ldots i_{\alpha+1}}\left(\Omega_{1}, \ldots, \Omega_{p}, \omega_{1}, \omega_{2}, \phi_{1}, \ldots, \phi_{u}\right)
\end{aligned} \\
& -\sum a_{h} X \operatorname{div}_{i_{\pi+1}} \ldots X \operatorname{div}_{i_{\gamma}} \\
& h \in H_{2}^{b, k,-} \times \operatorname{Switch}[C]_{g}^{h, i_{\pi+1} \ldots i_{\alpha+1}}\left(\Omega_{1}, \ldots, \Omega_{p}, \omega_{1}, \omega_{2}, \phi_{1}, \ldots, \phi_{u}\right) \\
& =\sum_{j \in J} a_{j} C_{g}^{j}\left(\Omega_{1}, \ldots, \Omega_{p}, \omega_{1}, \omega_{2}, \phi_{1}, \ldots, \phi_{u}\right) \text {, }
\end{aligned}
$$

where each $C_{g}^{j}$ has the factor $\nabla \omega_{1}$ contracting against the $D$-crucial factor and is simply subsequent to $\vec{\kappa}_{\text {simp }}$.

We now denote the $(u+1)$-simple character (the one defined by $\left.\nabla \phi_{1}, \ldots, \nabla \omega_{1}\right)$ of the tensor fields Hit $\operatorname{div}_{i_{\gamma}} C_{g}^{h, i_{\pi+1} \ldots i_{\alpha}, i_{\gamma}}\left(\Omega_{1}, \ldots, \Omega_{p}, \omega_{1}, \omega_{2}, \phi_{1}, \ldots, \phi_{u}\right)$ by $\vec{\kappa}_{\text {simp }}^{\prime}$. (Observe that they all have the same $(u+1)$-simple character.)

We observe that just applying Lemma 2.1 to (2-59) (all tensor fields are acceptable and have the same simple character $\vec{\kappa}_{\text {simp }}^{\prime}$ - we treat $\nabla \omega_{1}$ as a factor $\nabla \phi_{u+1}$ and the factor $\nabla \omega_{2}$ as a factor $\nabla Y$ ) and we then pick out the sublinear combination where there are $M$ factors $\nabla v$ contracting against $T_{k}$, we obtain

$$
\begin{aligned}
& \text { (2-60) } \sum_{h \in H_{2}^{a, *, k}} a_{h} \operatorname{Hit}_{\operatorname{div}_{i_{\gamma}}} C_{g}^{h, i_{\pi+1} \ldots i_{\alpha}}\left(\Omega_{1}, \ldots, \Omega_{p}, \omega_{1}, \omega_{2}, \phi_{1}, \ldots, \phi_{u}\right) \nabla_{i_{\pi+1}} v \ldots \nabla_{i_{\alpha}} v \\
& +\sum_{x \in X} a_{x} X \operatorname{div}_{i_{\alpha+1}} C_{g}^{x, i_{\pi+1} \ldots i_{\alpha}, i_{\alpha+1}}\left(\Omega_{1}, \ldots, \Omega_{p}, \omega_{1}, \omega_{2}, \phi_{1}, \ldots, \phi_{u}\right) \\
& \times \nabla_{i_{\pi+1}} v \ldots \nabla_{i_{\alpha}} v \\
& +\sum_{j \in J} a_{j} C_{g}^{j, i_{\pi+1} \ldots i_{\alpha}}\left(\Omega_{1}, \ldots, \Omega_{p}, \omega_{1}, \omega_{2}, \phi_{1}, \ldots, \phi_{u}\right) \nabla_{i_{\pi+1}} v \ldots \nabla_{i_{\alpha}} v=0,
\end{aligned}
$$

where the tensor fields indexed in $X$ are acceptable and have a $(u+1)$-simple character $\vec{\kappa}_{\text {simp }}^{\prime}$ and each $C^{j}$ is simply subsequent to $\vec{\kappa}_{\text {simp }}^{\prime}$.

Now, observe that if $M \geq \frac{3}{2}$ then we can apply the Eraser (from the Appendix in [A 2012]) to $\nabla \omega_{1}$ and the index it contracts against in the $D$-crucial factor and derive our conclusion as in Case A. 
The remaining cases are when $M=1, M=\frac{1}{2}$ and $M=0$. The first one is easier, so we proceed to show our claim in that case. The two subcases $M=\frac{1}{2}, M=0$ will be discussed in the next subsection.

In the case $M=1$, i.e., the $D$-crucial factor is of the form $\nabla^{(p)} \Omega_{h}$, then we cannot derive our claim, because of the possibility that some tensor fields indexed in $X$ above have $\nabla \omega_{1}$ contracting according to the pattern $\nabla_{i} \omega_{1} \nabla^{i j} \Omega_{h} \nabla_{j} \psi$, where $\psi=v$ or $\psi=\phi_{h}$. Therefore, in this setting, we first apply the eraser twice to remove the expression $\nabla_{i j}^{(2)} \Omega_{h} \nabla^{i} \psi \nabla^{j} \omega_{1}$ and then apply Corollary 2 from [A 2010] ${ }^{59}$ to (2-60) (observe that (2-60) now falls under the inductive assumption of Lemma 4.6 in [A 2010] since we have lowered the weight ${ }^{60}$ to obtain a new equation in the form (2-60), where each tensor field in $X$ has the factor $\nabla \omega_{1}$ contracting against a factor $\nabla^{(l)} \Omega_{h}, l \geq 3$. Then, applying the eraser as explained, we derive our Lemma 2.9 in this case.

The cases $M=\frac{1}{2}, M=0$. Notice that in these cases we must have $\alpha=\pi$, by virtue of the definition of maximal "measure" above. We will then prove our claim by proving a more general claim by induction, in the next subsection.

2D. The remaining cases of Lemma 2.9. We prove our claim in these cases via an induction. In order to give a detailed proof, we will restate our lemma hypothesis in this case (with a slight change of notation).

The hypothesis of the remaining cases of Lemma 2.9. Recall that we assume that

$$
\begin{aligned}
\sum_{x \in X_{a}} a_{x} X_{*} \operatorname{div}_{i_{1}} C_{g}^{x, i_{1}}\left(\Omega_{1}, \ldots, \Omega_{p}, \phi_{1}, \ldots, \phi_{u},\left[\omega_{1}, \omega_{2}\right]\right) \\
\quad+\sum_{x \in X_{b}} a_{x} X_{*} \operatorname{div}_{i_{1}} C_{g}^{x, i_{1}}\left(\Omega_{1}, \ldots, \Omega_{p}, \phi_{1}, \ldots, \phi_{u},\left[\omega_{1}, \omega_{2}\right]\right) \\
\quad+\sum_{j \in J} a_{j} C_{g}^{j}\left(\Omega_{1}, \ldots, \Omega_{p}, \phi_{1}, \ldots, \phi_{u}\right)=0
\end{aligned}
$$

holds modulo complete contractions of length $\geq \sigma+u+3$ ( $\sigma \geq 3$ - here $\sigma$ stands for $u+p-$ see the next equation). We denote the weight of the complete contractions in the above by $-K$. The tensor fields in the above equation are each in the form

$$
\begin{aligned}
& \operatorname{pcontr}\left(S_{*} \nabla^{\left(v_{1}\right)} R_{x_{1} j k l} \otimes \cdots \otimes S_{*} \nabla^{\left(v_{u}\right)} R_{x_{z} j^{\prime} k^{\prime} l^{\prime}}\right. \\
& \left.\quad \otimes \nabla^{\left(a_{1}\right)} \Omega_{1} \otimes \cdots \otimes \nabla^{\left(a_{p}\right)} \Omega_{p} \otimes\left[\nabla \omega_{1} \otimes \nabla \omega_{2}\right] \otimes \nabla^{x_{1}} \tilde{\phi}_{1} \otimes \cdots \otimes \nabla^{x_{u}} \tilde{\phi}_{z}\right) .
\end{aligned}
$$

\footnotetext{
${ }^{59}$ Recall that we showed in [A 2010] that this is a corollary of Lemma 4.6 in [A 2010], which we have now shown.

${ }^{60}$ There is no danger of falling under a "forbidden case" of Lemma 2.1 by weight considerations since we are assuming that none of the tensor fields of minimum rank in the assumption of Lemma 2.3 are "bad".
} 
We recall that the $u$-simple character of the above has been denoted by $\vec{\kappa}_{\text {simp. }}$. Recall that we are now assuming that all the factors $\nabla^{\left(a_{i}\right)} \Omega_{x}$ in $\vec{\kappa}_{\text {simp }}$ are acceptable. ${ }^{61}$ The complete contractions indexed in $J$ in (2-61) are simply subsequent to $\vec{\kappa}_{\text {simp. We }}$ also recall that $X_{*} \operatorname{div}_{i}$ stands for the sublinear combination in $X \operatorname{div}_{i}$ where $\nabla_{i}$ is not allowed to hit either of the factors $\nabla \omega_{1}, \nabla \omega_{2}$.

We recall that the tensor fields indexed in $X_{a}$ have the free index $i_{i_{1}}$ belonging to the factor $\nabla \omega_{1}$. The tensor fields indexed in $X_{b}$ have the free index $i_{1}$ not belonging to any of the factors $\nabla \omega_{1}, \nabla \omega_{2}$.

We recall the key assumption that for each of the tensor fields indexed in $X_{a}$, there is at least one removable index in each tensor field

$$
C_{g}^{x, i_{1}}\left(\Omega_{1}, \ldots, \Omega_{p}, \phi_{1}, \ldots, \phi_{u},\left[\omega_{1}, \omega_{2}\right]\right),
$$

$x \in X_{a}{ }^{62}$

In order to complete our proof of Lemma 2.9, we will show that we can write

$$
\begin{aligned}
& \begin{array}{l}
\sum_{x \in X_{a}} a_{x} C_{g}^{x, i_{1}}\left(\Omega_{1}, \ldots, \Omega_{p}, \phi_{1}, \ldots, \phi_{u},\left[\omega_{1}, \omega_{2}\right]\right) \nabla_{i_{1}} v \\
=\sum_{x \in X^{\prime}} a_{x} X_{*} \operatorname{div}_{i_{2}} \ldots X_{*} \operatorname{div}_{i_{a}} \\
\quad \times C_{g}^{x, i_{1} \ldots i_{a}}\left(\Omega_{1}, \ldots, \Omega_{p}, \phi_{1}, \ldots, \phi_{u},\left[\omega_{1}, \omega_{2}\right]\right) \nabla_{i_{1}} v \\
\quad+\sum_{j \in J} a_{j} C_{g}^{j}\left(\Omega_{1}, \ldots, \Omega_{p}, \phi_{1}, \ldots, \phi_{u}\right)
\end{array}
\end{aligned}
$$

where the tensor fields indexed in $X^{\prime}$ are acceptable in the form (2-62), each with rank $a \geq 2$. Note that this will imply the remaining cases of Lemma 2.9, completing the proof of Lemma 2.3.

We recall that we are proving this claim when the assumption (2-61) formally falls under our inductive assumption of Proposition 1.1 (if we formally treat $\nabla \omega_{1}$, $\nabla \omega_{2}$ as factors $\left.\nabla \phi_{z+1}, \nabla \phi_{z+2}\right)$.

We will prove (2-63) by inductively proving a more general statement.

Assumptions. We consider vector fields (that is, partial contractions with one free index)

$$
\begin{aligned}
& C_{g}^{\zeta, i_{1}}\left(\Omega_{1}, \ldots, \Omega_{b}, \phi_{1}, \ldots, \phi_{v}, Y, \psi_{1}, \ldots, \psi_{\tau}\right), \\
& C_{g}^{\zeta, i_{1}}\left(\Omega_{1}, \ldots, \Omega_{b}, \phi_{1}, \ldots, \phi_{v},\left[\chi_{1}, \chi_{2}\right], \psi_{1}, \ldots, \psi_{\tau}\right),
\end{aligned}
$$

${ }^{61}$ Meaning that each $a_{i} \geq 2$.

${ }^{62}$ Recall the definition of a "removable" index from page 8 . 
in the following forms, respectively,

$$
\begin{aligned}
\operatorname{pcontr}\left(S_{*}\right. & \nabla^{\left(v_{1}\right)} R_{x_{1} j k l} \otimes \cdots \otimes S_{*} \nabla^{\left(v_{v}\right)} R_{x_{v} j^{\prime} k^{\prime} l^{\prime}} \\
& \otimes \nabla^{\left(a_{1}\right)} \Omega_{1} \otimes \ldots \nabla^{\left(a_{b}\right)} \Omega_{b} \otimes \nabla Y \\
& \left.\otimes \nabla \psi_{1} \otimes \cdots \otimes \nabla \psi_{\tau} \otimes \nabla^{x_{1}} \tilde{\phi}_{1} \otimes \cdots \otimes \nabla^{x_{v}} \tilde{\phi}_{v}\right), \\
\operatorname{pcontr}\left(S_{*}\right. & \nabla^{\left(v_{1}\right)} R_{x_{1} j k l} \otimes \cdots \otimes S_{*} \nabla^{\left(v_{v}\right)} R_{x_{v} j^{\prime} k^{\prime} l^{\prime}} \\
& \otimes \nabla^{\left(a_{1}\right)} \Omega_{1} \otimes \ldots \nabla^{\left(a_{b}\right)} \Omega_{b} \otimes\left[\nabla \chi_{1} \otimes \nabla \chi_{2}\right] \\
& \left.\otimes \nabla \psi_{1} \otimes \cdots \otimes \nabla \psi_{\tau} \otimes \nabla^{x_{1}} \tilde{\phi}_{1} \otimes \cdots \otimes \nabla^{x_{v}} \tilde{\phi}_{v}\right),
\end{aligned}
$$

for which the weight is $-W+1, W \leq K$. We also assume $v+b \geq 2$. Note: the bracket [...] stands for the antisymmetrization of the indices ${ }_{a},{ }_{b}$ in the expression $\nabla_{a} \omega_{1} \nabla_{b} \omega_{2}$.

We assume (respectively) that

$$
\begin{aligned}
& \sum_{\zeta \in Z_{a}} a_{\zeta} X_{*} \operatorname{div}_{i_{1}} C_{g}^{\zeta, i_{1}}\left(\Omega_{1}, \ldots, \Omega_{b}, \phi_{1}, \ldots, \phi_{v}, Y, \psi_{1}, \ldots, \psi_{\tau}\right) \\
& +\sum_{\zeta \in \bar{Z}_{a}} a_{\zeta} X_{*} \operatorname{div}_{i_{1}} \ldots X_{*} \operatorname{div}_{i_{\gamma}} \\
& \quad \times C_{g}^{\zeta, i_{1} \ldots i_{\gamma}}\left(\Omega_{1}, \ldots, \Omega_{b}, \phi_{1}, \ldots, \phi_{v}, Y, \psi_{1}, \ldots, \psi_{\tau}\right) \\
& +\sum_{\zeta \in Z_{b}} a_{\zeta} X_{*} \operatorname{div}_{i_{1}} C_{g}^{\zeta, i_{1}}\left(\Omega_{1}, \ldots, \Omega_{b}, \phi_{1}, \ldots, \phi_{v}, Y, \psi_{1}, \ldots, \psi_{\tau}\right) \\
& +\sum_{j \in J} a_{j} C_{g}^{j}\left(\Omega_{1}, \ldots, \Omega_{b}, \phi_{1}, \ldots, \phi_{v}, Y, \psi_{1}, \ldots, \psi_{\tau}\right)=0,
\end{aligned}
$$

and

$$
\begin{aligned}
& \sum_{\zeta \in Z_{a}} a_{\zeta} X_{*} \operatorname{div}_{i_{1}} C_{g}^{\zeta, i_{1}}\left(\Omega_{1}, \ldots, \Omega_{b}, \phi_{1}, \ldots, \phi_{v},\left[\chi_{1}, \chi_{2}\right], \psi_{1}, \ldots, \psi_{\tau}\right) \\
& +\sum_{\zeta \in \bar{Z}_{a}} a_{\zeta} X_{*} \operatorname{div}_{i_{1}} \ldots X_{*} \operatorname{div}_{i_{\gamma}} \\
& \quad \times C_{g}^{\zeta, i_{1} \ldots i_{\gamma}}\left(\Omega_{1}, \ldots, \Omega_{b}, \phi_{1}, \ldots, \phi_{v},\left[\chi_{1}, \chi_{2}\right], \psi_{1}, \ldots, \psi_{\tau}\right) \\
& +\sum_{\zeta \in Z_{b}} a_{\zeta} X_{*} \operatorname{div}_{i_{1}} C_{g}^{\zeta, i_{1}}\left(\Omega_{1}, \ldots, \Omega_{b}, \phi_{1}, \ldots, \phi_{v},\left[\chi_{1}, \chi_{2}\right], \psi_{1}, \ldots, \psi_{\tau}\right) \\
& +\sum_{j \in J} a_{j} C_{g}^{j}\left(\Omega_{1}, \ldots, \Omega_{b}, \phi_{1}, \ldots, \phi_{v},\left[\chi_{1}, \chi_{2}\right], \psi_{1}, \ldots, \psi_{\tau}\right)=0,
\end{aligned}
$$

hold modulo complete contractions of length $\geq v+b+\tau+3$.

The tensor fields indexed in $Z_{a}$ are assumed to have the free index in one of the factors $\nabla Y, \nabla \psi_{1}, \ldots, \nabla \psi_{\tau}$, or one of the factors $\nabla \chi_{1}, \nabla \chi_{2}, \nabla \psi_{1}, \ldots, \nabla \psi_{\tau}$, respectively. The tensor fields indexed in $\bar{Z}_{a}$ have rank $\gamma \geq 2$ and all their free indices 
belong to the factors $\nabla Y, \nabla \psi_{1}, \ldots, \nabla \psi_{\tau}$, or the factors $\nabla \chi_{1}, \nabla \chi_{2}, \nabla \psi_{1}, \ldots, \nabla \psi_{\tau}$, respectively. The tensor fields indexed in $Z_{b}$ have the property that $i_{1}$ does not belong to any of the factors

$$
\nabla Y, \nabla \psi_{1}, \ldots, \nabla \psi_{\tau} \quad \text { or } \quad \nabla \chi_{1}, \nabla \chi_{2}, \nabla \psi_{1}, \ldots, \nabla \psi_{\tau},
$$

respectively. We also assume that for the tensor fields indexed in $Z_{a} \cup Z_{b} \cup \bar{Z}_{a}$, none of the factors $\nabla \psi_{1}, \ldots, \nabla \psi_{\tau}$ are contracting against a special index in any factor $S_{*} \nabla^{(v)} R_{i j k l}$ and none of them are contracting against the rightmost index in any $\nabla^{\left(a_{h}\right)} \Omega_{h}$ (we will refer to this property as the $\mathfrak{p}$-property). We assume that $v+b \geq 2$, and furthermore if $v+b=2$ then for each $\zeta \in Z_{a} \cup Z_{b}$, the factors $\nabla Y$ (or $\nabla \chi_{1}, \nabla \chi_{2}$ ) are also not contracting against a special index in any $S_{*} \nabla^{(v)} R_{i j k l}$ and are not contracting against the rightmost index in any $\nabla^{\left(a_{h}\right)} \Omega_{h}$. Finally (and importantly) we assume that for the tensor fields indexed in $Z_{a}$, there is at least one removable index in each $C^{\zeta, i_{1}}$. (In this setting, for a tensor field indexed in $Z_{a}$, a "removable" index is either a nonspecial index in a factor $S_{*} \nabla^{(v)} R_{i j k l}$, with $v>0$ or an index in a factor $\nabla^{(B)} \Omega_{h}, B \geq 3$.)

Convention. In this subsection only, for tensor fields in the forms (2-66), (2-67) we say then an index is special if it is one of the indices ${ }_{k}, l$ in a factor $S_{*} \nabla^{(v)} R_{i j k l}$ (this is the usual convention), or if it is an index in a factor $\nabla_{r_{1} \ldots r_{B}}^{(B)} \Omega_{h}$ for which all the other indices are contracting against factors $\nabla \psi_{1}, \ldots, \nabla \psi_{\tau}$.

All tensor fields in (2-66), (2-67) have a given $v$-simple character $\bar{\kappa}_{\text {simp }}$. We assume the complete contractions indexed in $J$ have a weak $v$-character Weak $\left(\bar{\kappa}_{\text {simp }}\right)$ and are simply subsequent to $\bar{\kappa}_{\text {simp. }}$. Here $X_{*} \operatorname{div}_{i}$ stands for the sublinear combination in $X \operatorname{div}_{i}$ where $\nabla_{i}$ is not allowed to hit any of the factors

$$
\nabla Y, \nabla \psi_{1}, \ldots, \nabla \psi_{\tau} \quad \text { or } \quad \nabla \chi_{1}, \nabla \chi_{2}, \nabla \psi_{1}, \ldots, \nabla \psi_{\tau}
$$

respectively.

The claims of the general statement. We claim that under the assumption (2-67), there exists a linear combination of acceptable 2-tensor fields in the form (2-64), (2-65) respectively (indexed in $W$ below), for which the p-property is satisfied, so that (respectively)

$$
\begin{aligned}
& \sum_{\zeta \in Z_{a}} a_{\zeta} C_{g}^{\zeta, i_{1}}\left(\Omega_{1}, \ldots, \Omega_{b}, \phi_{1}, \ldots, \phi_{v}, Y, \psi_{1}, \ldots, \psi_{\tau}\right) \nabla_{i_{1}} v \\
& -\sum_{w \in W} a_{w} X_{*} \operatorname{div}_{i_{2}} C_{g}^{w, i_{1} i_{2}}\left(\Omega_{1}, \ldots, \Omega_{b}, \phi_{1}, \ldots, \phi_{v}, Y, \psi_{1}, \ldots, \psi_{\tau}\right) \nabla_{i_{1}} v \\
& +\sum_{j \in J} a_{j} C_{g}^{j, i_{1}}\left(\Omega_{1}, \ldots, \Omega_{b}, \phi_{1}, \ldots, \phi_{v}, Y, \psi_{1}, \ldots, \psi_{\tau}\right) \nabla_{i_{1}} v=0,
\end{aligned}
$$


and

$$
\begin{aligned}
& \sum_{\zeta \in Z_{a}} a_{\zeta} C_{g}^{\zeta, i_{1}}\left(\Omega_{1}, \ldots, \Omega_{b}, \phi_{1}, \ldots, \phi_{v},\left[\chi_{1}, \chi_{2}\right], \psi_{1}, \ldots, \psi_{\tau}\right) \nabla_{i_{1}} v \\
& +\sum_{w \in W} a_{w} X_{*} \operatorname{div}_{i_{2}} \\
& \quad \times C_{g}^{w, i_{1} i_{2}}\left(\Omega_{1}, \ldots, \Omega_{b}, \phi_{1}, \ldots, \phi_{v},\left[\chi_{1}, \chi_{2}\right], \psi_{1}, \ldots, \psi_{\tau}\right) \nabla_{i_{1}} v \\
& +\sum_{j \in J} a_{j} C_{g}^{j, i_{1}}\left(\Omega_{1}, \ldots, \Omega_{b}, \phi_{1}, \ldots, \phi_{v},\left[\chi_{1}, \chi_{2}\right], \psi_{1}, \ldots, \psi_{\tau}\right) \nabla_{i_{1}} v=0 .
\end{aligned}
$$

We observe that when $\tau=0$ and $v+b \geq 3$, (2-69) coincides with (2-63). ${ }^{63}$ Therefore, if we can prove this general statement, we will have shown Lemma 2.9 in full generality, thus also completing the proof of Lemma 2.3.

We also have a further claim, when we assume (2-66), (2-67) with $v+b=2$. In that case, we also claim that we can write

$$
\begin{array}{r}
\sum_{\zeta \in Z_{a} \cup Z_{b} \cup \bar{Z}_{a}} X_{+} \operatorname{div}_{i_{1}} C_{g}^{\zeta, i_{1}}\left(\Omega_{1}, \ldots, \Omega_{b}, \phi_{1}, \ldots, \phi_{v}, Y, \psi_{1}, \ldots, \psi_{\tau}\right) \\
=\sum_{q \in Q} a_{q} X_{+} \operatorname{div}_{i_{1}} C_{g}^{q, i_{1}}\left(\Omega_{1}, \ldots, \Omega_{b}, \phi_{1}, \ldots, \phi_{v}, Y, \psi_{1}, \ldots, \psi_{\tau}\right) \\
\quad+\sum_{j \in J} a_{j} C_{g}^{j}\left(\Omega_{1}, \ldots, \Omega_{b}, \phi_{1}, \ldots, \phi_{v}, Y, \psi_{1}, \ldots, \psi_{\tau}\right),
\end{array}
$$

and

$$
\begin{array}{r}
\sum_{\zeta \in Z_{a} \cup \bar{Z}_{a} \cup Z_{b}} a_{+} \operatorname{div}_{i_{1}} C_{g}^{\zeta, i_{1}}\left(\Omega_{1}, \ldots, \Omega_{b}, \phi_{1}, \ldots, \phi_{v},\left[\chi_{1}, \chi_{2}\right], \psi_{1}, \ldots, \psi_{\tau}\right) \\
=\sum_{q \in Q} a_{q} X_{+} \operatorname{div}_{i_{1}} C_{g}^{q, i_{1}}\left(\Omega_{1}, \ldots, \Omega_{b}, \phi_{1}, \ldots, \phi_{v},\left[\chi_{1}, \chi_{2}\right], \psi_{1}, \ldots, \psi_{\tau}\right) \\
\quad+\sum_{j \in J} a_{j} C_{g}^{j}\left(\Omega_{1}, \ldots, \Omega_{b}, \phi_{1}, \ldots, \phi_{v},\left[\chi_{1}, \chi_{2}\right], \psi_{1}, \ldots, \psi_{\tau}\right),
\end{array}
$$

where the tensor fields indexed in $Q$ are in the same form as (2-64) or (2-65) respectively, but have a factor (expression) $\nabla^{(2)} Y$ or $\nabla_{a[i}^{(2)} \omega_{1} \nabla_{j]} \omega_{2}$, respectively, and satisfy all the other properties of the tensor fields in $Z_{a}$.

Consequence of (2-68), (2-69) when $v+b \geq 3$. We here codify an implication one can derive from (2-68), (2-69). This implication will be useful further down in this subsection. We see that by making the factors $\nabla v$ into $X_{*} \operatorname{div}$ in (2-66), ${ }^{64}(2-67)$

${ }^{63}$ Also, the assumption of existence of a non removable index coincides with the corresponding assumption of Lemma 2.3.

${ }^{64}$ See the Appendix in [A 2012]. 
and replacing into (2-68), (2-69), we obtain

$$
\begin{aligned}
& \sum_{\zeta \in Z_{a}^{\prime}} a_{\zeta} X_{*} \operatorname{div}_{i_{1}} C_{g}^{\zeta, i_{1}}\left(\Omega_{1}, \ldots, \Omega_{b}, \phi_{1}, \ldots, \phi_{v}, Y, \psi_{1}, \ldots, \psi_{\tau}\right) \\
& \quad+\sum_{\zeta \in Z_{b}} a_{\zeta} X_{*} \operatorname{div}_{i_{1}} C_{g}^{\zeta, i_{1}}\left(\Omega_{1}, \ldots, \Omega_{b}, \phi_{1}, \ldots, \phi_{v}, Y, \psi_{1}, \ldots, \psi_{\tau}\right) \\
& \quad+\sum_{j \in J} a_{j} C_{g}^{j}\left(\Omega_{1}, \ldots, \Omega_{b}, \phi_{1}, \ldots, \phi_{v}, Y, \psi_{1}, \ldots, \psi_{\tau}\right)=0
\end{aligned}
$$

and

$$
\begin{aligned}
& \sum_{\zeta \in Z_{a}^{\prime}} a_{\zeta} X_{*} \operatorname{div}_{i_{1}} C_{g}^{\zeta, i_{1}}\left(\Omega_{1}, \ldots, \Omega_{b}, \phi_{1}, \ldots, \phi_{v},\left[\chi_{1}, \chi_{2}\right], \psi_{1}, \ldots, \psi_{\tau}\right) \\
& \quad+\sum_{\zeta \in Z_{b}} a_{\zeta} X_{*} \operatorname{div}_{i_{1}} C_{g}^{\zeta, i_{1}}\left(\Omega_{1}, \ldots, \Omega_{b}, \phi_{1}, \ldots, \phi_{v},\left[\chi_{1}, \chi_{2}\right], \psi_{1}, \ldots, \psi_{\tau}\right) \\
& \quad+\sum_{j \in J} a_{j} C_{g}^{j}\left(\Omega_{1}, \ldots, \Omega_{b}, \phi_{1}, \ldots, \phi_{v},\left[\chi_{1}, \chi_{2}\right], \psi_{1}, \ldots, \psi_{\tau}\right)=0
\end{aligned}
$$

where here the tensor fields indexed in $Z_{a}^{\prime}$ are like the tensor fields indexed in $Z_{a}$ in (2-66), (2-67) but have the additional feature that no free index belongs to the factor $\nabla \psi_{1}$ (and all the other assumptions of equations (2-66), (2-67) continue to hold).

We then claim that we can derive new equations

$$
\begin{gathered}
\sum_{\zeta \in Z_{a}^{\prime}} a_{\zeta} X_{+} \operatorname{div}_{i_{1}} C_{g}^{\zeta, i_{1}}\left(\Omega_{1}, \ldots, \Omega_{b}, \phi_{1}, \ldots, \phi_{v}, Y, \psi_{1}, \ldots, \psi_{\tau}\right) \\
+\sum_{\zeta \in Z_{b}} a_{\zeta} X_{+} \operatorname{div}_{i_{1}} C_{g}^{\zeta, i_{1}}\left(\Omega_{1}, \ldots, \Omega_{b}, \phi_{1}, \ldots, \phi_{v}, Y, \psi_{1}, \ldots, \psi_{\tau}\right) \\
=\sum_{q \in Q} a_{q} X_{+} \operatorname{div}_{i_{1}} C_{g}^{q, i_{1}}\left(\Omega_{1}, \ldots, \Omega_{b}, \phi_{1}, \ldots, \phi_{v}, Y, \psi_{1}, \ldots, \psi_{\tau}\right) \\
+\sum_{j \in J} a_{j} C_{g}^{j}\left(\Omega_{1}, \ldots, \Omega_{b}, \phi_{1}, \ldots, \phi_{v}, Y, \psi_{1}, \ldots, \psi_{\tau}\right)
\end{gathered}
$$

and

$$
\begin{array}{r}
\sum_{\zeta \in Z_{a}^{\prime}} a_{\zeta} X_{+} \operatorname{div}_{i_{1}} C_{g}^{\zeta, i_{1}}\left(\Omega_{1}, \ldots, \Omega_{b}, \phi_{1}, \ldots, \phi_{v},\left[\chi_{1}, \chi_{2}\right], \psi_{1}, \ldots, \psi_{\tau}\right) \\
+\sum_{\zeta \in Z_{b}} a_{\zeta} X_{+} \operatorname{div}_{i_{1}} C_{g}^{\zeta, i_{1}}\left(\Omega_{1}, \ldots, \Omega_{b}, \phi_{1}, \ldots, \phi_{v},\left[\chi_{1}, \chi_{2}\right], \psi_{1}, \ldots, \psi_{\tau}\right) \\
=\sum_{q \in Q} a_{q} X_{+} \operatorname{div}_{i_{1}} C_{g}^{q, i_{1}}\left(\Omega_{1}, \ldots, \Omega_{b}, \phi_{1}, \ldots, \phi_{v},\left[\chi_{1}, \chi_{2}\right], \psi_{1}, \ldots, \psi_{\tau}\right) \\
+\sum_{j \in J} a_{j} C_{g}^{j}\left(\Omega_{1}, \ldots, \Omega_{b}, \phi_{1}, \ldots, \phi_{v},\left[\chi_{1}, \chi_{2}\right], \psi_{1}, \ldots, \psi_{\tau}\right),
\end{array}
$$


where here $X_{+} \operatorname{div}_{i}$ stands for the sublinear combination in $X \operatorname{div}_{i}$ where $\nabla_{i}$ is allowed to hit the factor $\nabla Y$ or $\nabla \chi_{1}$ (respectively), but not the factors $\nabla \psi_{1}, \ldots, \nabla \phi_{\tau}$, $\left(\nabla \chi_{2}\right)$. Furthermore, the linear combinations indexed in $Q$ stand for generic linear combinations of vector fields in the form (2-64) or (2-65), only with the expressions $\nabla Y$ or $\nabla_{[a} \omega_{1} \nabla_{b]} \omega_{2}$ replaced by expressions $\nabla^{(2)} Y, \nabla_{c[a}^{(2)} \omega_{1} \nabla_{b]} \omega_{2}$.

Proof that (2-74), (2-75) follow from (2-68), (2-69). We prove the above by an induction. We will first subdivide $Z_{a}^{\prime}, Z_{b}$ into subsets as follows: $\zeta \in Z_{a, \mathfrak{p}}^{\prime}$ or $\zeta \in Z_{b, \mathfrak{p}}$ if the factor $\nabla Y$ (or one of the factors $\nabla \chi_{1}, \nabla \chi_{2}$ ) contracts against a special index in the same factor against which $\nabla \psi_{1}$ contracts.

Now, if $Z_{a, \mathfrak{p}}^{\prime} \cup Z_{b, \mathfrak{p}} \neq \varnothing$ our inductive statement will be that we can write

$$
\begin{aligned}
& \begin{aligned}
\sum_{\zeta \in Z_{a, \mathfrak{p}}^{\prime}} a_{\zeta} X_{+} & \operatorname{div}_{i_{1}} \ldots X_{+} \operatorname{div}_{i_{\gamma}} \\
& \times C_{g}^{\zeta, i_{1} \ldots i_{\gamma}}\left(\Omega_{1}, \ldots, \Omega_{b}, \phi_{1}, \ldots, \phi_{v}, Y, \psi_{1}, \ldots, \psi_{\tau}\right)
\end{aligned} \\
& =\sum_{\zeta \in Z_{b, \mathfrak{p}}} a_{\zeta} X_{+} \operatorname{div}_{i_{1}} C_{g}^{\zeta, i_{1}}\left(\Omega_{1}, \ldots, \Omega_{b}, \phi_{1}, \ldots, \phi_{v}, Y, \psi_{1}, \ldots, \psi_{\tau}\right) \\
& +\sum_{t \in T^{k}} a_{t} X_{+} \operatorname{div}_{i_{1}} \ldots X_{+} \operatorname{div}_{i_{k}} \\
& \times C_{g}^{t, i_{1} \ldots i_{k}}\left(\Omega_{1}, \ldots, \Omega_{b}, \phi_{1}, \ldots, \phi_{v}, Y, \psi_{1}, \ldots, \psi_{\tau}\right) \\
& +\sum a_{\zeta} X_{+} \operatorname{div}_{i_{1}} \ldots X_{+} \operatorname{div}_{i_{\gamma}} \\
& \zeta \in Z_{a, N o p}^{\prime} \times C_{g}^{\zeta, i_{1} \ldots i_{\gamma}}\left(\Omega_{1}, \ldots, \Omega_{b}, \phi_{1}, \ldots, \phi_{v}, Y, \psi_{1}, \ldots, \psi_{\tau}\right) \\
& +\sum_{q \in Q} a_{q} X_{+} \operatorname{div}_{i_{1}} C_{g}^{q, i_{1}}\left(\Omega_{1}, \ldots, \Omega_{b}, \phi_{1}, \ldots, \phi_{v}, Y, \psi_{1}, \ldots, \psi_{\tau}\right) \\
& +\sum_{j \in J} a_{j} C_{g}^{j}\left(\Omega_{1}, \ldots, \Omega_{b}, \phi_{1}, \ldots, \phi_{v}, Y, \psi_{1}, \ldots, \psi_{\tau}\right),
\end{aligned}
$$

and

$$
\begin{aligned}
& \sum_{\zeta \in Z_{a, \mathfrak{p}}^{\prime}} a_{\zeta} X_{+} \operatorname{div}_{i_{1}} C_{g}^{\zeta, i_{1}}\left(\Omega_{1}, \ldots, \Omega_{b}, \phi_{1}, \ldots, \phi_{v},\left[\chi_{1}, \chi_{2}\right], \psi_{1}, \ldots, \psi_{\tau}\right) \\
& =\sum_{\zeta \in Z_{b, \mathfrak{p}}} a_{\zeta} X_{+} \operatorname{div}_{i_{1}} C_{g}^{\zeta, i_{1}}\left(\Omega_{1}, \ldots, \Omega_{b}, \phi_{1}, \ldots, \phi_{v},\left[\chi_{1}, \chi_{2}\right], \psi_{1}, \ldots, \psi_{\tau}\right) \\
& +\sum_{t \in T^{k}} a_{t} X_{+} \operatorname{div}_{i_{1}} \ldots X_{+} \operatorname{div}_{i_{k}} \\
& \quad \times C_{g}^{t, i_{1} \ldots i_{k}}\left(\Omega_{1}, \ldots, \Omega_{b}, \phi_{1}, \ldots, \phi_{v},\left[\chi_{1}, \chi_{2}\right], \psi_{1}, \ldots, \psi_{\tau}\right) \\
& +\sum_{\zeta \in Z_{a, N o p}^{\prime} a_{\zeta} X_{+}} \operatorname{div}_{i_{1}} \ldots X_{+} \operatorname{div}_{i_{\gamma}} \\
& \quad \times C_{g}^{\zeta, i_{1} \ldots i_{\gamma}}\left(\Omega_{1}, \ldots, \Omega_{b}, \phi_{1}, \ldots, \phi_{v},\left[\chi_{1}, \chi_{2}\right], \psi_{1}, \ldots, \psi_{\tau}\right)
\end{aligned}
$$




$$
\begin{aligned}
& +\sum_{q \in Q} a_{q} X_{+} \operatorname{div}_{i_{1}} C_{g}^{q, i_{1}}\left(\Omega_{1}, \ldots, \Omega_{b}, \phi_{1}, \ldots, \phi_{v},\left[\chi_{1}, \chi_{2}\right], \psi_{1}, \ldots, \psi_{\tau}\right) \\
& +\sum_{j \in J} a_{j} C_{g}^{j}\left(\Omega_{1}, \ldots, \Omega_{b}, \phi_{1}, \ldots, \phi_{v},\left[\chi_{1}, \chi_{2}\right], \psi_{1}, \ldots, \psi_{\tau}\right)
\end{aligned}
$$

where the tensor fields indexed in $T^{k}$ have all the properties of the tensor fields indexed in $Z_{a, \mathfrak{p}}^{\prime}$ (in particular the index in $\nabla \psi_{1}$ is not free) and in addition have rank $k$. The tensor fields indexed in $Z_{a, N o p}^{\prime}$ in the right-hand side have all the regular features of the terms indexed in $Z_{a}^{\prime}$ (in particular rank $\gamma \geq 1$ and the factor $\nabla \psi_{1}$ does not contain a free index) and in addition none of the factors $\nabla Y$ (or $\left.\nabla \chi_{1}, \nabla \chi_{2}\right)$ contract against a special index.

Our inductive claim is that we can write

$$
\begin{aligned}
& \sum_{\zeta \in Z_{a}^{\prime}} a_{\zeta} X_{+} \operatorname{div}_{i_{1}} C_{g}^{\zeta, i_{1}}\left(\Omega_{1}, \ldots, \Omega_{b}, \phi_{1}, \ldots, \phi_{v}, Y, \psi_{1}, \ldots, \psi_{\tau}\right) \\
& =\sum_{\zeta \in Z_{b}} a_{\zeta} X_{+} \operatorname{div}_{i_{1}} C_{g}^{\zeta, i_{1}}\left(\Omega_{1}, \ldots, \Omega_{b}, \phi_{1}, \ldots, \phi_{v}, Y, \psi_{1}, \ldots, \psi_{\tau}\right) \\
& \quad+\sum_{t \in T^{k+1}} a_{t} X_{+} \operatorname{div}_{i_{1}} \ldots X_{+} \operatorname{div}_{i_{k+1}} \\
& \quad \times C_{g}^{t, i_{1} \ldots i_{k+1}}\left(\Omega_{1}, \ldots, \Omega_{b}, \phi_{1}, \ldots, \phi_{v}, Y, \psi_{1}, \ldots, \psi_{\tau}\right) \\
& \quad+\sum_{\zeta \in Z_{a, N o p}^{\prime}} a_{\zeta} X_{+} \operatorname{div}_{i_{1}} \ldots C_{+}^{\zeta, i_{1} \ldots i_{\gamma}}\left(\Omega_{1}, \ldots, \Omega_{b}, \phi_{1}, \ldots, \phi_{v}, Y, \psi_{1}, \ldots, \psi_{\tau}\right) \\
& \quad+\sum_{q \in Q} a_{q} X_{+} \operatorname{div}_{i_{1}} C_{g}^{q, i_{1}}\left(\Omega_{1}, \ldots, \Omega_{b}, \phi_{1}, \ldots, \phi_{v}, Y, \psi_{1}, \ldots, \psi_{\tau}\right) \\
& \quad+\sum_{j \in J} a_{j} C_{g}^{j}\left(\Omega_{1}, \ldots, \Omega_{b}, \phi_{1}, \ldots, \phi_{v}, Y, \psi_{1}, \ldots, \psi_{\tau}\right),
\end{aligned}
$$

and

$$
\begin{aligned}
& \sum_{\zeta \in Z_{a}^{\prime}} a_{\zeta} X_{+} \operatorname{div}_{i_{1}} C_{g}^{\zeta, i_{1}}\left(\Omega_{1}, \ldots, \Omega_{b}, \phi_{1}, \ldots, \phi_{v},\left[\chi_{1}, \chi_{2}\right], \psi_{1}, \ldots, \psi_{\tau}\right) \\
& =\sum_{\zeta \in Z_{b}} a_{\zeta} X_{+} \operatorname{div}_{i_{1}} C_{g}^{\zeta, i_{1}}\left(\Omega_{1}, \ldots, \Omega_{b}, \phi_{1}, \ldots, \phi_{v},\left[\chi_{1}, \chi_{2}\right], \psi_{1}, \ldots, \psi_{\tau}\right) \\
& \quad+\sum_{t \in T^{k+1}} a_{t} X_{+} \operatorname{div}_{i_{1}} \ldots X_{+} \operatorname{div}_{i_{k}} \\
& \quad \times C_{g}^{t, i_{1} \ldots i_{k+1}}\left(\Omega_{1}, \ldots, \Omega_{b}, \phi_{1}, \ldots, \phi_{v},\left[\chi_{1}, \chi_{2}\right], \psi_{1}, \ldots, \psi_{\tau}\right) \\
& \quad+\sum_{\zeta \in Z_{a, N o p}^{\prime}} a_{\zeta} X_{+} \operatorname{div}_{i_{1}} \ldots X_{+} \operatorname{div}_{i_{\gamma}} \\
& \quad \times C_{g}^{\zeta, i_{1} \ldots i_{\gamma}}\left(\Omega_{1}, \ldots, \Omega_{b}, \phi_{1}, \ldots, \phi_{v},\left[\chi_{1}, \chi_{2}\right], \psi_{1}, \ldots, \psi_{\tau}\right)
\end{aligned}
$$




$$
\begin{aligned}
& +\sum_{q \in Q} a_{q} X_{+} \operatorname{div}_{i_{1}} C_{g}^{q, i_{1}}\left(\Omega_{1}, \ldots, \Omega_{b}, \phi_{1}, \ldots, \phi_{v},\left[\chi_{1}, \chi_{2}\right], \psi_{1}, \ldots, \psi_{\tau}\right) \\
& +\sum_{j \in J} a_{j} C_{g}^{j}\left(\Omega_{1}, \ldots, \Omega_{b}, \phi_{1}, \ldots, \phi_{v},\left[\chi_{1}, \chi_{2}\right], \psi_{1}, \ldots, \psi_{\tau}\right)=0 .
\end{aligned}
$$

We will derive (2-78), (2-79) momentarily. For now, we observe that by iterative repetition of the above inductive step we are reduced to showing (2-74), (2-75) under the additional assumption that $Z_{a, \mathfrak{p}}^{\prime}=\varnothing$.

Under that assumption, we denote by $Z_{b, \mathfrak{p}} \subset Z_{b}$ the index set of vector fields for which the factor $\nabla Y$ (or one of the factors $\nabla \chi_{1}, \nabla \chi_{2}$ ) contracts against a special index. We will then prove another inductive statement: We assume that we can write

$$
\begin{aligned}
& \sum_{\zeta \in Z_{b, \mathfrak{p}}} a_{\zeta} X_{+} \operatorname{div}_{i_{1}} C_{g}^{\zeta, i_{1}}\left(\Omega_{1}, \ldots, \Omega_{b}, \phi_{1}, \ldots, \phi_{v}, Y, \psi_{1}, \ldots, \psi_{\tau}\right) \\
& \begin{aligned}
=\sum_{t \in V^{k}} a_{t} X_{+} & \operatorname{div}_{i_{1}} \ldots X_{+} \operatorname{div}_{i_{k}} \\
\quad & \times C_{g}^{t, i_{1} \ldots i_{k}}\left(\Omega_{1}, \ldots, \Omega_{b}, \phi_{1}, \ldots, \phi_{v}, Y, \psi_{1}, \ldots, \psi_{\tau}\right)
\end{aligned} \\
& \quad+\sum_{\zeta \in Z_{b, N o p}} a_{\zeta} X_{+} \operatorname{div}_{i_{1}} C_{g}^{\zeta, i_{1}}\left(\Omega_{1}, \ldots, \Omega_{b}, \phi_{1}, \ldots, \phi_{v}, Y, \psi_{1}, \ldots, \psi_{\tau}\right) \\
& \quad+\sum_{q \in Q} a_{q} X_{+} \operatorname{div}_{i_{1}} C_{g}^{q, i_{1}}\left(\Omega_{1}, \ldots, \Omega_{b}, \phi_{1}, \ldots, \phi_{v}, Y, \psi_{1}, \ldots, \psi_{\tau}\right) \\
& \quad+\sum_{j \in J} a_{j} C_{g}^{j}\left(\Omega_{1}, \ldots, \Omega_{b}, \phi_{1}, \ldots, \phi_{v}, Y, \psi_{1}, \ldots, \psi_{\tau}\right),
\end{aligned}
$$

and

$$
\begin{aligned}
& \sum_{\zeta \in Z_{b, \mathfrak{p}}} a_{\zeta} X_{+} \operatorname{div}_{i_{1}} C_{g}^{\zeta, i_{1}}\left(\Omega_{1}, \ldots, \Omega_{b}, \phi_{1}, \ldots, \phi_{v},\left[\chi_{1}, \chi_{2}\right], \psi_{1}, \ldots, \psi_{\tau}\right) \\
& \begin{aligned}
\sum_{t \in V^{k}} a_{t} X_{+} \operatorname{div}_{i_{1}} \ldots X_{+} \operatorname{div}_{i_{k}} \\
\quad \times C_{g}^{t, i_{1} \ldots i_{k}}\left(\Omega_{1}, \ldots, \Omega_{b}, \phi_{1}, \ldots, \phi_{v},\left[\chi_{1}, \chi_{2}\right], \psi_{1}, \ldots, \psi_{\tau}\right)
\end{aligned} \\
& \quad+\sum_{\zeta \in Z_{b, N o p}} a_{\zeta} X_{+} \operatorname{div}_{i_{1}} C_{g}^{\zeta, i_{1}}\left(\Omega_{1}, \ldots, \Omega_{b}, \phi_{1}, \ldots, \phi_{v},\left[\chi_{1}, \chi_{2}\right], \psi_{1}, \ldots, \psi_{\tau}\right) \\
& \quad+\sum_{q \in Q} a_{q} X_{+} \operatorname{div}_{i_{1}} C_{g}^{q, i_{1}}\left(\Omega_{1}, \ldots, \Omega_{b}, \phi_{1}, \ldots, \phi_{v},\left[\chi_{1}, \chi_{2}\right], \psi_{1}, \ldots, \psi_{\tau}\right) \\
& \quad+\sum_{j \in J} a_{j} C_{g}^{j}\left(\Omega_{1}, \ldots, \Omega_{b}, \phi_{1}, \ldots, \phi_{v},\left[\chi_{1}, \chi_{2}\right], \psi_{1}, \ldots, \psi_{\tau}\right),
\end{aligned}
$$

where the tensor fields indexed in $V^{k}$ have all the features of the tensor fields indexed in $Z_{b, \mathfrak{p}}$ but in addition have all the $k$ free indices not belonging to factors 
$\nabla \psi_{1}, \ldots, \nabla \psi_{\tau}$. The tensor fields indexed in $Z_{b, N o p}$ have all the regular features of the tensor fields in $Z_{b}$ and in addition have the factor $\nabla Y$ (or the factors $\nabla \chi_{1}, \nabla \chi_{2}$ ) not contracting against special indices. The terms indexed in $Q$ are as required in the right-hand side of (2-74), (2-75) (which are the equations that we are proving).

We will then show that we can write

$$
\begin{aligned}
& \sum_{\zeta \in Z_{b, p}} a_{\zeta} X_{+} \operatorname{div}_{i_{1}} C_{g}^{\zeta, i_{1}}\left(\Omega_{1}, \ldots, \Omega_{b}, \phi_{1}, \ldots, \phi_{v}, Y, \psi_{1}, \ldots, \psi_{\tau}\right) \\
& \begin{aligned}
=\sum_{t \in V^{k+1}} a_{t} & X_{+} \operatorname{div}_{i_{1}} \ldots X_{+} \operatorname{div}_{i_{k+1}} \\
& \times C_{g}^{t, i_{1} \ldots i_{k+1}}\left(\Omega_{1}, \ldots, \Omega_{b}, \phi_{1}, \ldots, \phi_{v}, Y, \psi_{1}, \ldots, \psi_{\tau}\right)
\end{aligned} \\
& +\sum_{\zeta \in Z_{b, N o p}} a_{\zeta} X_{+} \operatorname{div}_{i_{1}} C_{g}^{\zeta, i_{1}}\left(\Omega_{1}, \ldots, \Omega_{b}, \phi_{1}, \ldots, \phi_{v}, Y, \psi_{1}, \ldots, \psi_{\tau}\right) \\
& +\sum_{q \in Q} a_{q} X_{+} \operatorname{div}_{i_{1}} C_{g}^{q, i_{1}}\left(\Omega_{1}, \ldots, \Omega_{b}, \phi_{1}, \ldots, \phi_{v}, Y, \psi_{1}, \ldots, \psi_{\tau}\right) \\
& +\sum_{j \in J} a_{j} C_{g}^{j}\left(\Omega_{1}, \ldots, \Omega_{b}, \phi_{1}, \ldots, \phi_{v}, Y, \psi_{1}, \ldots, \psi_{\tau}\right),
\end{aligned}
$$

and

$$
\begin{aligned}
& \sum_{\zeta \in Z_{b, \mathfrak{p}}} a_{\zeta} X_{+} \operatorname{div}_{i_{1}} C_{g}^{\zeta, i_{1}}\left(\Omega_{1}, \ldots, \Omega_{b}, \phi_{1}, \ldots, \phi_{v},\left[\chi_{1}, \chi_{2}\right], \psi_{1}, \ldots, \psi_{\tau}\right) \\
& =\sum_{t \in V^{k+1}} a_{t} X_{+} \operatorname{div}_{i_{1}} \ldots X_{+} \operatorname{div}_{i_{k+1}} \\
& \times C_{g}^{t, i_{1} \ldots i_{k+1}}\left(\Omega_{1}, \ldots, \Omega_{b}, \phi_{1}, \ldots, \phi_{v},\left[\chi_{1}, \chi_{2}\right], \psi_{1}, \ldots, \psi_{\tau}\right) \\
& +\sum_{\zeta \in Z_{b, N o p}} a_{\zeta} X_{+} \operatorname{div}_{i_{1}} C_{g}^{\zeta, i_{1}}\left(\Omega_{1}, \ldots, \Omega_{b}, \phi_{1}, \ldots, \phi_{v},\left[\chi_{1}, \chi_{2}\right], \psi_{1}, \ldots, \psi_{\tau}\right) \\
& +\sum_{q \in Q} a_{q} X_{+} \operatorname{div}_{i_{1}} C_{g}^{q, i_{1}}\left(\Omega_{1}, \ldots, \Omega_{b}, \phi_{1}, \ldots, \phi_{v},\left[\chi_{1}, \chi_{2}\right], \psi_{1}, \ldots, \psi_{\tau}\right) \\
& +\sum_{j \in J} a_{j} C_{g}^{j}\left(\Omega_{1}, \ldots, \Omega_{b}, \phi_{1}, \ldots, \phi_{v},\left[\chi_{1}, \chi_{2}\right], \psi_{1}, \ldots, \psi_{\tau}\right) .
\end{aligned}
$$

(Here the tensor fields indexed in $V^{k+1}$ have all the features described above and moreover have rank $k+1$.)

Thus, by iterative repetition of this step we are reduced to showing our claim under the additional assumption that $Z_{a, \mathfrak{p}}^{\prime}=Z_{b, \mathfrak{p}}=\varnothing$.

We prove (2-82), (2-83) below. Now, we present the rest of our claims under the assumption that $Z_{a, \mathfrak{p}}^{\prime}=Z_{b, \mathfrak{p}}=\varnothing$. For the rest of this proof we may assume that all tensor fields have the factor $\nabla Y$ (or the factors $\nabla \chi_{1}, \nabla \chi_{2}$ ) not contracting against special indices. 
We then perform a new induction: We assume that we can write

$$
\begin{aligned}
& \sum_{\zeta \in Z_{a}^{\prime}} a_{\zeta} X_{+} \operatorname{div}_{i_{1}} C_{g}^{\zeta, i_{1}}\left(\Omega_{1}, \ldots, \Omega_{b}, \phi_{1}, \ldots, \phi_{v}, Y, \psi_{1}, \ldots, \psi_{\tau}\right) \\
& =\sum_{\zeta \in Z_{b}} a_{\zeta} X_{+} \operatorname{div}_{i_{1}} C_{g}^{\zeta, i_{1}}\left(\Omega_{1}, \ldots, \Omega_{b}, \phi_{1}, \ldots, \phi_{v}, Y, \psi_{1}, \ldots, \psi_{\tau}\right) \\
& \quad+\sum_{t \in T^{k}} a_{t} X_{+} \operatorname{div}_{i_{1}} \ldots X_{+} \operatorname{div}_{i_{k}} \\
& \quad \times C_{g}^{t, i_{1} \ldots i_{k}}\left(\Omega_{1}, \ldots, \Omega_{b}, \phi_{1}, \ldots, \phi_{v}, Y, \psi_{1}, \ldots, \psi_{\tau}\right) \\
& \quad+\sum_{q \in Q} a_{q} X_{+} \operatorname{div}_{i_{1}} C_{g}^{q, i_{1}}\left(\Omega_{1}, \ldots, \Omega_{b}, \phi_{1}, \ldots, \phi_{v}, Y, \psi_{1}, \ldots, \psi_{\tau}\right) \\
& \quad+\sum_{j \in J} a_{j} C_{g}^{j}\left(\Omega_{1}, \ldots, \Omega_{b}, \phi_{1}, \ldots, \phi_{v}, Y, \psi_{1}, \ldots, \psi_{\tau}\right),
\end{aligned}
$$

and

$$
\begin{aligned}
& \sum_{\zeta \in Z_{a}^{\prime}} a_{\zeta} X_{+} \operatorname{div}_{i_{1}} C_{g}^{\zeta, i_{1}}\left(\Omega_{1}, \ldots, \Omega_{b}, \phi_{1}, \ldots, \phi_{v},\left[\chi_{1}, \chi_{2}\right], \psi_{1}, \ldots, \psi_{\tau}\right) \\
& =\sum_{\zeta \in Z_{b}} a_{\zeta} X_{+} \operatorname{div}_{i_{1}} C_{g}^{\zeta, i_{1}}\left(\Omega_{1}, \ldots, \Omega_{b}, \phi_{1}, \ldots, \phi_{v},\left[\chi_{1}, \chi_{2}\right], \psi_{1}, \ldots, \psi_{\tau}\right) \\
& \quad+\sum_{t \in T^{k}} a_{t} X_{+} \operatorname{div}_{i_{1}} \ldots X_{+} \operatorname{div}_{i_{k}} \\
& \quad \times C_{g}^{t, i_{1} \ldots i_{k}}\left(\Omega_{1}, \ldots, \Omega_{b}, \phi_{1}, \ldots, \phi_{v},\left[\chi_{1}, \chi_{2}\right], \psi_{1}, \ldots, \psi_{\tau}\right) \\
& \quad+\sum_{q \in Q} a_{q} X_{+} \operatorname{div}_{i_{1}} C_{g}^{q, i_{1}}\left(\Omega_{1}, \ldots, \Omega_{b}, \phi_{1}, \ldots, \phi_{v},\left[\chi_{1}, \chi_{2}\right], \psi_{1}, \ldots, \psi_{\tau}\right) \\
& \quad+\sum_{j \in J} a_{j} C_{g}^{j}\left(\Omega_{1}, \ldots, \Omega_{b}, \phi_{1}, \ldots, \phi_{v},\left[\chi_{1}, \chi_{2}\right], \psi_{1}, \ldots, \psi_{\tau}\right),
\end{aligned}
$$

where the tensor fields indexed in $T^{k}$ have all the properties of the tensor fields indexed in $Z_{a}^{\prime}$ (in particular the index in $\nabla \psi_{1}$ is not free) and in addition have rank $k$. We then show that we can write

$$
\begin{aligned}
& \sum_{\zeta \in Z_{a}^{\prime}} a_{\zeta} X_{+} \operatorname{div}_{i_{1}} C_{g}^{\zeta, i_{1}}\left(\Omega_{1}, \ldots, \Omega_{b}, \phi_{1}, \ldots, \phi_{v}, Y, \psi_{1}, \ldots, \psi_{\tau}\right) \\
& =\sum_{\zeta \in Z_{b}} a_{\zeta} X_{+} \operatorname{div}_{i_{1}} C_{g}^{\zeta, i_{1}}\left(\Omega_{1}, \ldots, \Omega_{b}, \phi_{1}, \ldots, \phi_{v}, Y, \psi_{1}, \ldots, \psi_{\tau}\right) \\
& \quad+\sum_{t \in T^{k+1}} a_{t} X_{+} \operatorname{div}_{i_{1}} \ldots X_{+} \operatorname{div}_{i_{k+1}} \\
& \times C_{g}^{t, i_{1} \ldots i_{k}}\left(\Omega_{1}, \ldots, \Omega_{b}, \phi_{1}, \ldots, \phi_{v}, Y, \psi_{1}, \ldots, \psi_{\tau}\right)
\end{aligned}
$$




$$
\begin{aligned}
& +\sum_{q \in Q} a_{q} X_{+} \operatorname{div}_{i_{1}} C_{g}^{q, i_{1}}\left(\Omega_{1}, \ldots, \Omega_{b}, \phi_{1}, \ldots, \phi_{v}, Y, \psi_{1}, \ldots, \psi_{\tau}\right) \\
& +\sum_{j \in J} a_{j} C_{g}^{j}\left(\Omega_{1}, \ldots, \Omega_{b}, \phi_{1}, \ldots, \phi_{v}, Y, \psi_{1}, \ldots, \psi_{\tau}\right)
\end{aligned}
$$

and

$$
\begin{aligned}
& \sum_{\zeta \in Z_{a}^{\prime}} a_{\zeta} X_{+} \operatorname{div}_{i_{1}} C_{g}^{\zeta, i_{1}}\left(\Omega_{1}, \ldots, \Omega_{b}, \phi_{1}, \ldots, \phi_{v},\left[\chi_{1}, \chi_{2}\right], \psi_{1}, \ldots, \psi_{\tau}\right) \\
& =\sum_{\zeta \in Z_{b}} a_{\zeta} X_{+} \operatorname{div}_{i_{1}} C_{g}^{\zeta, i_{1}}\left(\Omega_{1}, \ldots, \Omega_{b}, \phi_{1}, \ldots, \phi_{v},\left[\chi_{1}, \chi_{2}\right], \psi_{1}, \ldots, \psi_{\tau}\right) \\
& \quad+\sum_{t \in T^{k+1}} a_{t} X_{+} \operatorname{div}_{i_{1}} \ldots X_{+} C_{g}^{t, i_{1} \ldots i_{k+1}}\left(\Omega_{1}, \ldots, \Omega_{b}, \phi_{1}, \ldots, \phi_{v},\left[\chi_{1}, \chi_{2}\right], \psi_{1}, \ldots, \psi_{\tau}\right) \\
& \quad+\sum_{q \in Q} a_{q} X_{+} \operatorname{div}_{i_{1}} C_{g}^{q, i_{1}}\left(\Omega_{1}, \ldots, \Omega_{b}, \phi_{1}, \ldots, \phi_{v},\left[\chi_{1}, \chi_{2}\right], \psi_{1}, \ldots, \psi_{\tau}\right) \\
& \quad+\sum_{j \in J} a_{j} C_{g}^{j}\left(\Omega_{1}, \ldots, \Omega_{b}, \phi_{1}, \ldots, \phi_{v},\left[\chi_{1}, \chi_{2}\right], \psi_{1}, \ldots, \psi_{\tau}\right) .
\end{aligned}
$$

We will derive (2-86), (2-87) momentarily. For now, we observe that by iterative repetition of the above we are reduced to showing (2-74), (2-75) under the further assumption that $Z_{a}^{\prime}=\varnothing$. In that setting, we can just repeatedly apply the eraser (see the Appendix in [A 2012] for a definition of this notion) to as many factors $\nabla \psi_{\tau}$ as needed in order to reduce ourselves to a new true equation where each of the real factors contracts against at most one of the factors $\nabla \psi_{1}, \ldots, \nabla \psi_{\tau}, \nabla Y$ (or $\left.\nabla \chi_{1}, \nabla \chi_{2}\right){ }^{65}$ Then, by invoking Corollary 1 from [A 2010] ${ }^{66}$ and then reintroducing the factors we erased, we derive our claim.

Proof of (2-86) and (2-87). Picking out the sublinear combination in (2-84), (2-85) with one derivative on $\nabla Y$ or $\nabla \chi_{1}$ and substituting into (2-72), (2-73) we derive

$$
\begin{gathered}
\sum_{t \in T^{k}} a_{t} X_{*} \operatorname{div}_{i_{1}} \ldots X_{*} \operatorname{div}_{i_{k}} C_{g}^{t, i_{1} \ldots i_{k}}\left(\Omega_{1}, \ldots, \Omega_{b}, \phi_{1}, \ldots, \phi_{v}, Y, \psi_{1}, \ldots, \psi_{\tau}\right) \\
+\sum_{\zeta \in Z_{b}} a_{\zeta} X_{*} \operatorname{div}_{i_{1}} C_{g}^{\zeta, i_{1}}\left(\Omega_{1}, \ldots, \Omega_{b}, \phi_{1}, \ldots, \phi_{v}, Y, \psi_{1}, \ldots, \psi_{\tau}\right) \\
=\sum_{j \in J} a_{j} C_{g}^{j}\left(\Omega_{1}, \ldots, \Omega_{b}, \phi_{1}, \ldots, \phi_{v}, Y, \psi_{1}, \ldots, \psi_{\tau}\right),
\end{gathered}
$$

${ }^{65}$ All remaining factors $\nabla \psi_{1}, \ldots, \nabla \psi_{\tau}$ and also the factor(s) $\nabla Y$ (or $\nabla \chi_{1}, \nabla \chi_{2}$ ) are treated as factors $\nabla \phi_{h}$.

${ }^{66}$ Notice that there will necessarily be at least one nonsimple factor $S_{*} \nabla^{(v)} R_{i j k l}$ or $\nabla^{(B)} \Omega_{h}$, by virtue of the factors $\nabla Y$ (or $\nabla \omega_{1}, \nabla \omega_{2}$ ), therefore that corollary can be applied. 
and

$$
\begin{aligned}
& \sum_{t \in T^{k}} a_{t} X_{*} \operatorname{div}_{i_{1}} \ldots X_{*} \operatorname{div}_{i_{k}} \\
& \quad \times C_{g}^{t, i_{1} \ldots i_{k}}\left(\Omega_{1}, \ldots, \Omega_{b}, \phi_{1}, \ldots, \phi_{v},\left[\chi_{1}, \chi_{2}\right], \psi_{1}, \ldots, \psi_{\tau}\right) \\
& +\sum_{\zeta \in Z_{b}} a_{\zeta} X_{*} \operatorname{div}_{i_{1}} C_{g}^{\zeta, i_{1}}\left(\Omega_{1}, \ldots, \Omega_{b}, \phi_{1}, \ldots, \phi_{v},\left[\chi_{1}, \chi_{2}\right], \psi_{1}, \ldots, \psi_{\tau}\right) \\
& \quad=\sum_{j \in J} a_{j} C_{g}^{j}\left(\Omega_{1}, \ldots, \Omega_{b}, \phi_{1}, \ldots, \phi_{v},\left[\chi_{1}, \chi_{2}\right], \psi_{1}, \ldots, \psi_{\tau}\right) ;
\end{aligned}
$$

the sublinear combination $\sum_{\zeta \in Z_{b}} \ldots$ above is generic.

Split the index set $T^{k}$ according to which of the factors $\nabla \psi_{2}, \ldots, \nabla \psi_{\tau}, \nabla Y$ (or $\left.\nabla \psi_{1}, \ldots, \nabla \psi_{\tau}, \nabla \chi_{1}\right)$ contain the $k$ free indices. Thus we write $T^{k}=\bigcup_{\alpha \in A} T^{k, \alpha}$ (each $\alpha \in A$ corresponds to a $k$-subset of the set of factors $\nabla \psi_{1}, \ldots, \nabla \psi_{\tau}, \nabla Y$ or $\left.\nabla \psi_{1}, \ldots, \nabla \psi_{\tau}, \nabla \chi_{1}\right)$. We will then show that for each $\alpha \in A$ there exists a linear combination $\sum_{b \in B^{\alpha}} a_{b} C_{g}^{b, i_{1} \ldots i_{k+1}}$ of partial contractions in the form (2-64) or (2-65) with the first $k$ free indices belonging to the factors in the set $\alpha$, and the free index $i_{k+1}$ not belonging to $\nabla \psi_{1}$, so that

$$
\begin{array}{r}
\sum_{t \in T^{k, \alpha}} a_{t} C_{g}^{t, i_{1} \ldots i_{k}}\left(\Omega_{1}, \ldots, \Omega_{b}, \phi_{1}, \ldots, \phi_{v}, Y, \psi_{1}, \ldots, \psi_{\tau}\right) \nabla_{i_{1}} v \ldots \nabla_{i_{k}} v \\
-X_{*} \operatorname{div}_{i_{k+1}} \sum_{b \in B^{\alpha}} a_{b} C_{g}^{b, i_{1} \ldots i_{k+1}}\left(\Omega_{1}, \ldots, \Omega_{b}, \phi_{1}, \ldots, \phi_{v}, Y, \psi_{1}, \ldots, \psi_{\tau}\right) \\
\times \nabla_{i_{1}} v \ldots \nabla_{i_{k}} v \\
=\sum_{j \in J} a_{j} C_{g}^{j, i_{1} \ldots i_{k}}\left(\Omega_{1}, \ldots, \Omega_{b}, \phi_{1}, \ldots, \phi_{v}, Y, \psi_{1}, \ldots, \psi_{\tau}\right) \\
\times \nabla_{i_{1}} v \ldots \nabla_{i_{k}} v
\end{array}
$$

and

$$
\begin{array}{r}
\sum_{t \in T^{k, \alpha}} a_{t} C_{g}^{t, i_{1} \ldots i_{k}}\left(\Omega_{1}, \ldots, \Omega_{b}, \phi_{1}, \ldots, \phi_{v},\left[\chi_{1}, \chi_{2}\right], \psi_{1}, \ldots, \psi_{\tau}\right) \\
\times \nabla_{i_{1}} v \ldots \nabla_{i_{k}} v \\
-X_{*} \operatorname{div}_{i_{k+1}} \sum_{b \in B^{\alpha}} a_{b} C_{g}^{b, i_{1} \ldots i_{k+1}}\left(\Omega_{1}, \ldots, \Omega_{b}, \phi_{1}, \ldots, \phi_{v},\left[\chi_{1}, \chi_{2}\right], \psi_{1}, \ldots, \psi_{\tau}\right) \\
\times \nabla_{i_{1}} v \ldots \nabla_{i_{k}} v \\
=\sum_{j \in J} a_{j} C_{g}^{j, i_{1} \ldots i_{k}}\left(\Omega_{1}, \ldots, \Omega_{b}, \phi_{1}, \ldots, \phi_{v},\left[\chi_{1}, \chi_{2}\right], \psi_{1}, \ldots, \psi_{\tau}\right) \\
\times \nabla_{i_{1}} v \ldots \nabla_{i_{k}} v .
\end{array}
$$

If we can show the above for every $\alpha \in A$, then replacing the factor $\nabla v$ by $X_{+} \operatorname{div}$ we can derive our claim (2-86), (2-87).

Proof of (2-90) and (2-91). Refer to (2-88) and (2-89). Denote $Y$ or $\chi_{1}$ by $\psi_{\tau+1}$ for uniformity. We pick out any $\alpha \in A$; assume that $\alpha=\left\{\nabla \psi_{x_{1}}, \ldots, \nabla \psi_{x_{k}}\right\}$. 
Pick out the sublinear combination where the factors $\nabla \psi_{x_{1}}, \ldots, \nabla \psi_{x_{k}}$ which belong to $\alpha$ contract against the same factor as $\nabla \psi_{1}$. This sublinear combination $Z_{g}$ vanishes separately (that is, $Z_{g}=0$ ). We then apply the eraser to the factors $\nabla \psi_{2}, \ldots, \nabla Y \in A$ (notice this is well-defined, since all the above factors and the factor $\nabla \psi_{1}$ contract against nonspecial indices). We obtain a new true equation, which we denote by $\operatorname{Erase}\left[Z_{g}\right]=0$. It then follows that Erase $\left[Z_{g}\right]$. $\left(\nabla_{i_{1}} \psi_{x_{1}} \nabla^{i_{1}} v \ldots \nabla_{i_{k}} \psi_{x_{k}} \nabla^{i_{k}} v\right)=0$ is our desired conclusion (2-90), (2-91).

Sketch of proof of (2-78), (2-79), (2-82), (2-83). These equations can be proven by only a slight modification of the idea above. We again subdivide the index sets $T^{k}, V^{k}$ according to the set of factors $\nabla \psi_{2}, \ldots, \nabla \psi_{\tau}$ or $\nabla \psi_{2}, \ldots, \nabla \psi_{\tau}, \nabla \omega_{1}$ that contain the $k$ free indices (so we write $T^{k}=\bigcup_{\alpha \in A} T^{k, \alpha}$ and $V^{k}=\bigcup_{\alpha \in A} V^{k, \alpha}$ ) and we prove the claims above separately for those sublinear combinations.

To prove this, we pick out the sublinear combination in our hypotheses with the factors $\nabla \psi_{h}, h \in \alpha$ contracting against the same factor against which $\nabla \psi_{1}$ and $\nabla Y$ (or $\nabla \psi_{1}$ and $\nabla \omega_{1}$ ) are contracting. Say $\alpha=\left\{h_{1}, \ldots, h_{k}\right\}$; we then formally replace the expressions

$$
\begin{aligned}
& S_{*} \nabla_{r_{1} \ldots r_{\mu} l_{1} \ldots l_{k}}^{(v)} R_{i j k l} \nabla_{l_{1}} \psi_{h_{1}} \ldots \nabla^{l_{k}} \psi_{h_{k}} \nabla^{i} \tilde{\phi}_{1} \nabla^{j} \psi_{1} \nabla^{k} Y \text { or } \\
& \nabla_{r_{1} \ldots r_{\mu} l_{1} \ldots l_{k} s t}^{(A)} \Omega_{1} \nabla^{l_{1}} \psi_{h_{1}} \ldots \nabla^{l_{k}} \psi_{h_{k}} \nabla^{s} \psi_{1} \nabla^{t} Y
\end{aligned}
$$

and so on by expressions

$$
S_{*} \nabla_{r_{1} \ldots r_{\mu}}^{(\nu-k)} R_{i j k l} \nabla^{i} \tilde{\phi}_{1} \nabla^{j} \psi_{1} \nabla^{k} Y \quad \text { or } \quad \nabla_{r_{1} \ldots r_{\mu} s t}^{(A-k)} \Omega_{1} \nabla^{s} \psi_{1} \nabla^{t} Y
$$

and derive our claims (2-78), (2-79), (2-82), (2-83) as above.

Proof of the claims of our general statement: Equations (2-68) and (2-69). We will prove these claims by an induction. Our inductive assumptions are that (2-68), (2-69) follow from (2-66), (2-67) for any weight $-W^{\prime}, W^{\prime}<K$ and when $W^{\prime}=K$ they hold for any length $v+b \geq \gamma \geq 2$. We will then show the claim when the weight is $-K$, and $v+b=\gamma+1$. In the end, we will check our claims for the base case $v+b=2$.

Proof of the inductive step. Refer back to (2-66), (2-67). We will prove this claim in four steps.

Step 1: First, we will denote by $Z_{a}^{\text {spec }}, \bar{Z}_{a}^{\text {spec }}, Z_{b}^{\text {spec }}$ the index sets of the tensor fields for which $\nabla Y$ or one of the factors $\nabla \chi_{1}, \nabla \chi_{2}$ (respectively) contracts against a special index. Then using the inductive assumptions of our general claim, we will show that there exists a linear combination of 2-tensor fields (indexed in $W$ below) 
which satisfies all the requirements of (2-66), (2-68) so that

$$
\begin{aligned}
\sum_{\zeta \in Z_{a}^{\text {spec }}} a_{\zeta} C_{g}^{\zeta, i_{1}} \nabla_{i_{1}} v-X_{*} \operatorname{div}_{i_{2}} \sum_{w \in W} a_{w} C_{g}^{w, i_{1} i_{2}} \nabla_{i_{1}} v \\
=\sum_{\zeta \in Z_{a}^{O K}} a_{\zeta} C_{g}^{\zeta, i_{1}} \nabla_{i_{1}} v+\sum_{j \in J} a_{j} C_{g}^{j, i_{1}} \nabla_{i_{1}} v,
\end{aligned}
$$

where the tensor fields $\mathrm{n} Z_{a}^{O K}$ are generic linear combinations of tensor fields of the same general type as the ones indexed in $Z_{a}$ in (2-66), (2-68) and where in addition none of the factors $\nabla Y$ or $\nabla \chi_{1}, \nabla \chi_{2}$ contract against a special index.

Thus, if we can show the above, by replacing $\nabla v$ by an $X_{*} \operatorname{div}_{i}$, and substituting back into (2-66), (2-68), we are reduced to showing (2-67), (2-69) under the additional assumption that $Z_{a}^{s p e c}=\varnothing$.

Step 2: Then, under the assumption that $Z_{a}^{\text {spec }}=\varnothing$, we will show that we can write

$$
\begin{aligned}
\sum_{\zeta \in Z_{b}^{\text {spec }}} a_{\zeta} X_{*} \operatorname{div}_{i_{1}} C_{g}^{\zeta, i_{1}} & +\sum_{\zeta \in \bar{Z}_{a}^{\text {spec }}} a_{\zeta} X_{*} \operatorname{div}_{i_{1}} \ldots X_{*} \operatorname{div}_{i_{c}} C_{g}^{\zeta, i_{1} \ldots i_{c}} \\
& =X_{*} \operatorname{div}_{i_{1}} \ldots X_{*} \operatorname{div}_{i_{b}} \sum_{c \in C} a_{c} C_{g}^{c, i_{1} \ldots i_{b}}+\sum_{j \in J} a_{j} C_{g}^{j, i_{1}},
\end{aligned}
$$

where the tensor fields on the right-hand side are of the general form as the ones indexed in $Z_{b}, \bar{Z}_{a}$ in our hypothesis, and moreover the factors $\nabla Y$ (or the factors $\left.\nabla \chi_{1}, \nabla \chi_{2}\right)$ are not contracting against special indices.

Notice that if we can show (2-92), (2-93) then we are reduced to showing our claim under the additional assumption that for each $\zeta \in Z_{a} \cup \bar{Z}_{a} \cup Z_{b}$ the factors $\nabla Y$ (or $\nabla \chi_{1}, \nabla \chi_{2}$ ) are not contracting against special indices. We will show (2-92), (2-93) below.

Proof of (2-67), (2-69) under the assumption that for each $\zeta \in Z_{a} \cup \bar{Z}_{a} \cup Z_{b}$ the factors $\nabla Y$ or $\left(\nabla \chi_{1}, \nabla \chi_{2}\right)$ do not contract against special indices.

Step 3: Proof of (2-94) below. We note that for all the tensor fields in the rest of this proof will not have the factor $\nabla Y$ (or any of the factors $\nabla \chi_{1}, \nabla \chi_{2}$ ) contracting against a special index in any factor $S_{*} \nabla^{(\nu)} R_{i j k l}$ or $\nabla^{(B)} \Omega_{h}$. Now, we arbitrarily pick out one factor $T=S_{*} \nabla^{(v)} R_{i j k l}$ or $T=\nabla^{(B)} \Omega_{x}$ in $\bar{\kappa}_{\text {simp }}$ and call it the "chosen factor" for the rest of this subsection.

We will say that the factor $\nabla Y$ (or $\nabla \omega_{2}$ ) contracts against a good index in $T$, if it contracts against a nonspecial index in $T$ when $T$ is of the form $S_{*} \nabla^{(v)} R_{i j k l}$ with $v>0$; when $T$ is of the form $\nabla^{(B)} \Omega_{x}$, then it contracts against a good index provided $B \geq 3$.

We will say that the factor $\nabla Y$ (or $\nabla \omega_{2}$ ) contracts against a bad index if it contracts against the index ${ }_{j}$ in a factor $T=S_{*} R_{i j k l}$ or an index in a factor $T=\nabla^{(2)} \Omega_{x}$. We denote by $Z_{a}^{B A D} \subset Z_{a}$ the index set of tensor fields for which $\nabla Y$ (or $\nabla \omega_{2}$ ) 
contracts against a bad index. We also denote by $Z_{b}^{B A D} \subset Z_{b}$ the index set of the vector fields for which $\nabla Y$ contracts against a bad index in $T$ and $T$ also contains a free index. We will show that we can write

$$
\begin{aligned}
\sum_{\zeta \in Z_{a}^{B A D \cup Z_{b}^{B A D}}} a_{\zeta} C_{g}^{\zeta, i_{1}} \nabla_{i_{1}} v-X_{*} \operatorname{div}_{i_{2}} \sum_{h \in H} a_{h} C_{g}^{i_{1} i_{2}} \nabla_{i_{1}} v & \\
& =\sum_{\substack{\zeta \in Z_{a}^{\prime G O O D} \cup Z_{b}^{\prime G O O D}\\
}} a_{\zeta} C_{g}^{\zeta, i_{1}} \nabla_{i_{1}} v+\sum_{j \in J} a_{j} C_{g}^{j},
\end{aligned}
$$

where all the tensor fields indexed in $Z_{a}^{\prime G O O D} \cup Z_{b}^{\prime G O O D}$ are generic vector fields of the forms indexed in $Z_{a}, Z_{b}$, only with the factors $\nabla Y$ or $\nabla \omega_{2}$ contracting against a good index in the factor $T$. The tensor fields indexed in $H$ are as required in the claim of our general statement (they correspond to the index set $W$ in our general statement).

Step 4: Proof that (2-94) implies our claims (2-68), (2-69). We start by proving (2-94) (that is, we prove Step 3). Then, we will show how we can derive our claim from (2-94) (that is, we then prove Step 4).

Proof of Step 3: Proof of (2-94). We can prove this equation by virtue of our inductive assumption on our general claim. First, we define $\bar{Z}_{a}^{B A D} \subset \bar{Z}_{a}$ to stand for the index set of tensor fields where the factor $\nabla Y\left(\right.$ or $\left.\nabla \omega_{2}\right)$ is contracting against a bad index in the chosen factor. We pick out the sublinear combination in our lemma assumption where $\nabla Y$ (or $\nabla \omega_{2}$ ) are contracting against the chosen factor $T=S_{*} R_{i j k l}$ or $T=\nabla^{(2)} \Omega_{x}$ ). This sublinear combination must vanish separately, and we thus derive that

$$
\begin{aligned}
\sum_{\zeta \in Z_{a}^{B A D} \cup Z_{b}^{B A D}} a_{\zeta} X_{* *} \operatorname{div}_{i_{1}} C_{g}^{\zeta, i_{1}}+\sum_{\zeta \in \bar{Z}_{a}^{B A D}} a_{\zeta} X_{* *} \operatorname{div}_{i_{1}} \ldots X_{* *} \operatorname{div}_{i_{c}} C_{g}^{\zeta, i_{1} \ldots i_{c}} \\
+\sum_{\zeta \in Z_{b}^{n v B A D}} a_{f} C_{g}^{f, i_{1}}=\sum_{j \in J} a_{j} C_{g}^{j},
\end{aligned}
$$

where $X_{* *} \operatorname{div}_{i_{1}}$ stands for the sublinear combination for which $\nabla_{i_{1}}$ is not allowed to hit the chosen factor $T$. $Z_{b}^{n v B A D} \subset Z_{b}$ stands for the index set of tensor fields indexed in $Z_{b}$ with the free index $i_{1}$ not belonging to the chosen factor and also with the factor $\nabla Y$ (or $\nabla \omega_{2}$ ) contracting against a bad index.

Now, define an operation $\mathrm{Op}[\ldots]$ that acts on the complete contractions above by formally replacing any expression $\nabla_{i j}^{(2)} \Omega_{x} \nabla^{i} Y$ (or $\nabla_{i j}^{(2)} \Omega_{x} \nabla^{i} \chi_{2}$ ) by $\nabla_{j} D(D$ is a scalar function), or any expression $S_{*} R_{i j k l} \nabla^{i} \tilde{\phi}_{1} \nabla^{j} Y$ (or $S_{*} R_{i j k l} \nabla^{i} \tilde{\phi}_{1} \nabla^{j} \chi_{2}$ ) by $\nabla_{[k} \theta_{1} \nabla_{l]} \theta_{2}$. (Denote by $\tilde{\kappa}_{\text {simp }}$ the simple character of these resulting vector fields.) 
Acting on (2-95) by Op[...] produces a true equation, which we may write out as

$$
\begin{aligned}
& \sum_{\zeta \in Z_{a}^{B A D} \cup Z_{b}^{B A D}} a_{\zeta} X_{* *} \operatorname{div}_{i_{1}} \mathrm{Op}[C]_{g}^{\zeta, i_{1}}+X_{* *} \operatorname{div}_{i_{1}} \sum_{f \in F} a_{f} C_{g}^{f, i_{1}} \\
&+\sum_{\zeta \in \bar{Z}_{a}^{B A D}} a_{\zeta} X_{* *} \operatorname{div}_{i_{1}} \ldots X_{* *} \operatorname{div}_{i_{c}} C_{g}^{\zeta, i_{1} \ldots i_{c}}=\sum_{j \in J} a_{j} C_{g}^{j} .
\end{aligned}
$$

Here $X_{* *} \operatorname{div}_{i}$ stands for the sublinear combination in $\operatorname{div}_{i}$ where $\nabla_{i}$ is not allowed to hit the factor to which $\nabla_{i}$ belongs, nor any of the factors $\nabla \phi_{1}, \ldots, \nabla \phi_{u}$, $\nabla \psi_{1}, \ldots, \nabla \psi_{\tau}$, nor any of the factors $\nabla D, \nabla \theta_{1}, \nabla \theta_{2}$. The vector fields indexed in $F$ are generic vector fields with a simple character $\tilde{\kappa}_{\text {simp }}$, for which the free index $i_{i_{1}}$ does not belong to any of the factors $\nabla \psi_{1}, \ldots, \nabla \psi_{\tau}$ or any of the factors $\nabla D,\left(\nabla \chi_{1}\right), \nabla \theta_{1}, \nabla \theta_{2}$.

Now, observe that the above equation falls under our inductive assumption of the general statement we are proving: We now either have factors

$$
\begin{aligned}
& \nabla \psi_{1}, \ldots, \nabla \psi_{\tau}, \nabla D, \quad \text { or } \\
& \nabla \psi_{1}, \ldots, \nabla \psi_{\tau}, \nabla \chi_{1}, \nabla D, \quad \text { or } \\
& \nabla \psi_{1}, \ldots, \nabla \psi_{\tau},\left[\nabla \theta_{1}, \nabla \theta_{2}\right], \quad \text { or } \\
& \nabla \psi_{1}, \ldots, \nabla \psi_{\tau}, \nabla \chi_{1},\left[\nabla \theta_{1}, \nabla \theta_{2}\right] .
\end{aligned}
$$

Notice that the tensor fields indexed in $H_{a}^{B A D}, H_{b}^{B A D}$ are precisely the ones that contain a free index in one of these factors. Therefore, by our inductive assumption of the "general claim" we derive that there exists a linear combination of 2-tensor fields, $\sum_{v \in V} \ldots$, (with factors $\nabla \psi_{1}, \ldots, \nabla \psi_{\tau}, \nabla D$ and so on, and which satisfy the $\mathfrak{p}$-property for the factors $\left.\nabla \psi_{1}, \ldots, \nabla \psi_{\tau}\right)$ so that

$$
\sum_{\zeta \in Z_{a}^{B A D} \cup Z_{b}^{B A D}} a_{\zeta} \mathrm{Op}[C]_{g}^{\zeta, i_{1}} \nabla_{i_{1}} v-X_{* *} \operatorname{div}_{i_{2}} \sum_{v \in V} a_{v} C_{g}^{v, i_{1} i_{2}} \nabla_{i_{1}} v=\sum_{j \in J} a_{j} C_{g}^{j, i_{1}} \nabla_{i_{1}} v
$$

Now, we define an operation $\mathrm{Op}^{-1}[\ldots]$ that acts on the complete contractions in the above equation by replacing the factor $\nabla_{j} D$ by an expression $\nabla_{i j} \Omega_{x} \nabla^{j} Y$ (or $\nabla_{i j} \Omega_{x} \nabla^{j} \omega_{2}$ ), or by replacing the expression $\nabla_{[a} \theta_{1} \nabla_{b]} \theta_{2}$ by $S_{*} R_{i j a b} \nabla^{i} \tilde{\phi}_{1} \nabla^{j} Y$ (or $\left.S_{*} R_{i j a b} \nabla^{i} \tilde{\phi}_{1} \nabla^{j} \omega_{2}\right)$. The operation $\mathrm{Op}^{-1}$ clearly produces a true equation, which is our desired conclusion, (2-94).

Proof of Step 4. We derive our conclusions (2-68), (2-69) in pieces. First, we show these equations with the sublinear combinations $Z_{a}$ replaced by the index set $Z_{a, \text { spec }}$, which index the terms with the free index $i_{i_{1}}$ belonging to the factor $\nabla Y$ or $\nabla \omega_{1}$ (this will be Substep A). After proving this claim, we will show (2-68), (2-69) under the additional assumption that $Z_{a, \text { spec }}=\varnothing$ (this will be Substep B). 
Proof of Substep A. We make the $\nabla v$ s into $X_{*}$ divs in (2-94) and insert the resulting equations into our lemma hypothesis. We thus derive a new equation

$$
\begin{aligned}
\sum_{\zeta \in Z_{a}} a_{\zeta} X_{*} \operatorname{div}_{i_{1}} C_{g}^{\zeta, i_{1}} & +\sum_{\zeta \in Z_{b}^{1}} a_{\zeta} X_{*} \operatorname{div}_{i_{1}} C_{g}^{\zeta, i_{1}} \\
& +\sum_{\zeta \in Z_{b}^{2}} a_{\zeta} X_{*} \operatorname{div}_{i_{1}} \ldots X_{*} \operatorname{div}_{i_{a}} C_{g}^{\zeta, i_{1} \ldots i_{a}}+\sum_{j \in J} a_{j} C_{g}^{j}=0
\end{aligned}
$$

where we now have that the tensor fields indexed in $Z_{a}$ have a free index among the factors $\nabla \psi_{1}, \ldots, \nabla \psi_{\tau}, \nabla Y$ (or $\nabla \psi_{1}, \ldots, \nabla \psi_{\tau}, \nabla \chi_{1}, \nabla \chi_{2}$ ), and furthermore the factors $\nabla Y$ (or the factors $\nabla \omega_{1}, \nabla \omega_{2}$ ) are not contracting against a bad index in the chosen factor $T$. The tensor fields indexed in $Z_{b}^{1}$ have a free index that does not belong to one of the factors $\nabla \psi_{1}, \ldots, \nabla \psi_{\tau}, \nabla Y$ (or $\nabla \psi_{1}, \ldots, \nabla \psi_{\tau}, \nabla \chi_{1}, \nabla \chi_{2}$ ), and furthermore if the factor $\nabla Y$ (or one of the factors $\nabla \omega_{1}, \nabla \omega_{2}$ ) is contracting against a bad index in the chosen factor $T$, then $T$ does not contain the free index $i_{1}$. Finally the tensor fields indexed in $Z_{b}^{2}$ each have rank $a \geq 2$ and all free indices belong to the factors $\nabla \psi_{1}, \ldots, \nabla \psi_{\tau}, \nabla Y,\left(\nabla \omega_{1}, \nabla \omega_{2}\right)$. We may then rewrite (2-98) in the form

$$
\begin{aligned}
\sum_{\zeta \in Z_{a}} a_{\zeta} X_{*} \operatorname{div}_{i_{1}} C_{g}^{\zeta, i_{1}} & +\sum_{\zeta \in Z_{b}^{1}} a_{\zeta} X_{*} \operatorname{div}_{i_{1}} C_{g}^{\zeta, i_{1}} \\
& +\sum_{\zeta \in Z_{b}^{2^{\prime}}} a_{\zeta} X_{*} \operatorname{div}_{i_{1}} \ldots X_{*} \operatorname{div}_{i_{a}} C_{g}^{\zeta, i_{1} \ldots i_{a}}+\sum_{j \in J} a_{j} C_{g}^{j}=0,
\end{aligned}
$$

where now for the tensor fields indexed in $Z_{b}^{2^{\prime}}$, each $a \geq 1$ and the factor $\nabla \psi_{1}$ does not contain a free index for any of the tensor fields for which $\nabla Y$ (or one of $\left.\nabla \omega_{1}, \nabla \omega_{2}\right)$ contracts against a bad index in the chosen factor.

We will denote by $Z_{b, \sharp}^{1} \subset Z_{b}^{1}$ and $Z_{b, \sharp}^{2} \subset Z_{b}^{2^{\prime}}$ the index sets of tensor fields where $\nabla Y$ (or one of $\nabla \omega_{1}, \nabla \omega_{2}$ ) contracts against a bad index in the chosen factor $T$.

From (2-99) we derive

$$
\begin{aligned}
\sum_{\zeta \in Z_{b, \sharp}^{1}} a_{\zeta} X_{* *} \operatorname{div}_{i_{1}} C_{g}^{\zeta, i_{1}}+\sum_{\zeta \in Z_{b, \sharp}^{2}{ }^{\prime}} a_{\zeta} X_{* *} \operatorname{div}_{i_{1}} \ldots X_{* *} \operatorname{div}_{i_{a}} C_{g}^{\zeta, i_{1} \ldots i_{a}} & \\
& +\sum_{j \in J} a_{j} C_{g}^{j}=0,
\end{aligned}
$$

where $X_{* *} \operatorname{div}_{i}$ stands for the sublinear combination in $X_{*} \operatorname{div}_{i}$ for which $\nabla_{i}$ is in addition no allowed to hit the chosen factor $T$.

Then, applying operation Op as in Step 3 and the inductive assumption of the general claim we are proving, ${ }^{67}$ and then using the operation $\mathrm{Op}^{-1}[\ldots]$ as in the

${ }^{67}$ The resulting equation falls under the inductive assumption, as in Step 3. 
proof of Step 3, we derive

$$
\begin{aligned}
\sum_{\zeta \in Z_{b, \sharp}^{1}} a_{\zeta} X_{*} \operatorname{div}_{i_{1}} C_{g}^{\zeta, i_{1}}+\sum_{\zeta \in Z_{b, \sharp}^{2}{ }^{\prime}} a_{\zeta} X_{*} \operatorname{div}_{i_{1}} \ldots X_{*} \operatorname{div}_{i_{a}} C_{g}^{\zeta, i_{1} \ldots i_{a}} \\
=\sum_{\zeta \in Z_{O K}} a_{\zeta} X_{*} \operatorname{div}_{i_{1}} \ldots X_{*} \operatorname{div}_{i_{a}} C_{g}^{\zeta, i_{1} \ldots i_{a}}+\sum_{j \in J} a_{j} C_{g}^{j}=0,
\end{aligned}
$$

where the tensor fields indexed in $Z_{O K}$ have rank $a \geq 1$ (no free indices belonging to factors $\nabla \psi_{1}, \ldots, \nabla Y$ or $\left.\nabla \psi_{1}, \ldots, \nabla \chi_{2}\right)$ and furthermore have the property that the one index in $\nabla Y$ or $\nabla \omega_{1}$ does not contract against a bad index in the chosen factor (and it is also not free). Thus, replacing the above back into (2-99), we derive

$$
\begin{aligned}
\sum_{\zeta \in Z_{a}} a_{\zeta} X_{*} \operatorname{div}_{i_{1}} C_{g}^{\zeta, i_{1}}+\sum_{\zeta \in Z_{b}^{1^{\prime}}} a_{\zeta} X_{*} \operatorname{div}_{i_{1}} C_{g}^{\zeta, i_{1}} \\
+\sum_{\zeta \in Z_{b}^{2^{\prime \prime}}} a_{\zeta} X_{*} \operatorname{div}_{i_{1}} \ldots X_{*} \operatorname{div}_{i_{a}} C_{g}^{\zeta, i_{1} \ldots i_{a}}+\sum_{j \in J} a_{j} C_{g}^{j}=0
\end{aligned}
$$

where the tensor fields indexed in $Z_{b}^{1^{\prime}}, Z_{b}^{2^{\prime \prime}}$ have the additional restriction that if the factor $\nabla Y$ (or $\nabla \omega_{1}, \nabla \omega_{2}$ ) is contracting against the chosen factor $T$ then it is not contracting against a bad index in $T$.

We are now in a position to derive Substep A from the above: To see this claim, we just apply Erase $_{\nabla Y}$ or Erase $\overline{\nabla \omega}_{1}$ to (2-102) and multiply the resulting equation by $\nabla_{i_{1}} Y \nabla^{i_{1}} v$.

Substep B: Now, we are reduced to showing our claim when $Z_{a, s p e c}=\varnothing$. In that setting, we denote by $Z_{a, s} \subset Z_{a}$ the index set of vector fields in $Z_{a}$ for which the free index $i_{1}$ belongs to the factor $\nabla \psi_{s}$; we prove our claim separately for each of the sublinear combinations $\sum_{\zeta \in Z_{a, s}} \ldots$ This claim is proven by picking out the sublinear combinations in (2-66), (2-67) where the factors $\nabla \psi_{s}$ and $\nabla Y$ (or $\nabla \chi_{1}$ ) contract against the same factor, ${ }^{68}$ we then apply the eraser to $\nabla \psi_{s}$ (this is welldefined and produces a true equation), and multiply by $\nabla_{i_{1}} \psi_{s} \nabla^{i_{1}} v$. The resulting equation is precisely our claim for the sublinear combination $\sum_{\zeta \in Z_{a, s}} \ldots$

Sketch of the proof of Steps 1 and 2: Equations (2-92) and (2-93). We will sketch the proof of these claims for the sublinear combinations in $Z_{a}^{\text {spec }} \cup Z_{b}^{\text {spec }} \cup \bar{Z}_{\text {spec }}^{a}$ where one of the special indices in $C^{\zeta, i_{1}}$ is an index ${ }_{k}$ or $l$ that belongs to a factor $S_{*} \nabla^{(v)} R_{i j k l}$. The remaining case (where the special indices belong to factors $\nabla^{(a)} \Omega_{h}$ ) can be seen by a similar (simpler) argument. ${ }^{69}$

${ }^{68}$ These sublinear combinations vanish separately.

${ }^{69}$ The only extra feature in this setting is that one must prove the claim by a separate induction on the number of factors $\nabla \psi_{z}$ that are contracting against $\nabla^{(a)} \Omega_{h}$. 
For each $\zeta \in Z_{a}^{\text {spec }} \cup Z_{b}^{\text {spec }} \cup \bar{Z}_{\text {spec }}^{a}$, we denote by $\bar{C}_{g}^{\zeta, i_{1}}, \bar{C}_{g}^{\zeta, i_{1} \ldots i_{\gamma}}$ the tensor fields that arise from $C^{\zeta, i_{1}}, C_{g}^{\zeta, i_{1} \ldots i_{\gamma}}$ in (2-66), (2-68) by replacing the expressions

$$
S_{*} \nabla_{r_{1} \ldots r_{v}}^{(v)} R_{i j k l} \nabla^{i} \tilde{\phi}_{1} \nabla^{k} Y \quad \text { and } \quad S_{*} \nabla_{r_{1} \ldots r_{v}}^{(v)} R_{i j k l} \nabla^{i} \tilde{\phi}_{1} \nabla^{k} \chi_{2}
$$

with a factor $\nabla_{r_{1} \ldots r_{\nu j l}}^{(v+2)} \Omega_{b+1}$. We denote by $\tilde{\kappa}_{\text {simp }}$ the resulting simple character. We derive

(2-103) $\sum_{\zeta \in Z_{a}^{\text {spec }} \cup Z_{b}^{\text {spec }}} a_{\xi} X_{*} \operatorname{div}_{i_{1}} \bar{C}_{g}^{\zeta, i_{1}}+\sum_{\zeta \in \bar{Z}_{a}^{\text {spec }}} a_{\zeta} X_{*} \operatorname{div}_{i_{1}} \ldots X_{*} \operatorname{div}_{i_{\gamma}} \bar{C}_{g}^{\zeta, i_{1}}+\sum_{j \in J} a_{j} \bar{C}_{g}^{j}=0$.

Now, again applying the inductive assumption of our general statement to the above, we derive that there is a linear combination of tensor fields (indexed in $W$ below) with a free index $i_{1}$ belonging to one of the factors $\nabla \psi_{1}, \ldots, \nabla \psi_{\tau}$ or $\nabla \psi_{1}, \ldots, \nabla \psi_{\tau}, \nabla \chi_{1}$ so that

$$
\sum_{\zeta \in Z_{a}^{\text {spec }}} a_{\zeta} \bar{C}_{g}^{\zeta, i_{1}} \nabla_{i_{1}} v-X_{*} \operatorname{div}_{i_{2}} \sum_{w \in W} a_{w} C_{g}^{w, i_{1} i_{2}} \nabla_{i_{1}} v=\sum_{j \in J} a_{j} \bar{C}_{g}^{j} .
$$

Now, by applying an operation $\mathrm{Op}^{*}$ to the above which formally replaces the factor $\nabla_{r_{1} \ldots r_{A}}^{(A)} \Omega_{x}$ with a factor

$$
S_{*} \nabla_{r_{1} \ldots r_{A-2}}^{(A-2)} R_{i r_{A-1} k r_{A}} \nabla^{i} \tilde{\phi}_{1} \nabla^{k} Y \quad \text { or } \quad S_{*} \nabla_{r_{1} \ldots r_{A-2}}^{(A-2)} R_{i r_{A-1} k r_{A}} \nabla^{i} \tilde{\phi}_{1} \nabla^{k} \chi_{2},
$$

we derive (2-92) (since we can repeat the permutations by which (2-104) is made to hold formally, modulo introducing correction terms that allowed in the right-hand side of (2-92)).

We will now prove (2-93) (that is, Step 2) by repeating the induction performed in the "Consequence" we derived above (where we showed that inductively assuming (2-76), (2-77) we can derive (2-78), (2-79)):

We will show the claim of Step 2 in pieces: First consider the tensor fields indexed in $\bar{Z}_{a, \mathfrak{p}}$ of minimum rank 2 (denote the corresponding index set by $\bar{Z}_{a, \mathfrak{p}}^{2}$ ); we then show that we can write

$$
\begin{aligned}
& \sum_{\zeta \in \bar{Z}_{a, \mathfrak{p}}^{2}} a_{\zeta} X \operatorname{div}_{i_{1}} X \operatorname{div}_{i_{2}} C_{g}^{\zeta, i_{1} i_{2}}=\sum_{\zeta \in \bar{Z}_{a, \mathfrak{p}}^{3}} a_{\zeta} X \operatorname{div}_{i_{1}} \ldots X \operatorname{div}_{i_{3}} C_{g}^{\zeta, i_{1} \ldots i_{3}} \\
& \quad+\sum_{\zeta \in Z_{b, \mathfrak{p}}} a_{\zeta} X \operatorname{div}_{i_{1}} C_{g}^{\zeta, i_{1}}+\sum_{\zeta \in Z_{O K}} a_{\zeta} X \operatorname{div}_{i_{1}} \ldots X \operatorname{div}_{i_{a}} C_{g}^{\zeta, i_{1} \ldots i_{a}}+\sum_{j \in} a_{j} C^{j}
\end{aligned}
$$

The tensor fields indexed in $\bar{Z}_{a, \mathfrak{p}}^{3}, Z_{b, \mathfrak{p}}$ on the right-hand side are generic linear combinations in those forms (the first with rank 3 ). The tensor fields indexed in $Z_{O K}$ are generic linear combinations as allowed in the right-hand side of (2-93). Assuming we can prove (2-105), we are then reduced to showing our claim when the minimum rank among the tensor fields indexed in $Z_{a, \mathfrak{p}}$ is 3 . We may then 
"forget" about any $X \operatorname{div}_{i_{h}}$ where $i_{h}$ belongs to the factor $\nabla \psi_{1}$. Therefore, we are reduced to showing our claim when the minimum rank is 2 and the factor $\nabla \psi_{1}$ does not contain a free index. We then claim our claim by an induction (for the rest of this derivation, all tensor fields will not have a free index in the factor $\nabla \psi_{1}$ ): Assume that the minimum rank of the tensor fields indexed in $\bar{Z}_{a, \mathfrak{p}}$ is $k$, and they are indexed in $\bar{Z}_{a, \mathfrak{p}}^{k}$. We then show that we can write

$$
\begin{aligned}
& \sum_{\zeta \in \bar{Z}_{a, \mathfrak{p}}^{k}} a_{\zeta} X \operatorname{div}_{i_{1}} \ldots X \operatorname{div}_{i_{k}} C_{g}^{\zeta, i_{1} \ldots i_{k}}=\sum_{\zeta \in \bar{Z}_{a, \mathfrak{p}}^{k+1}} a_{\zeta} X \operatorname{div}_{i_{1}} \ldots X \operatorname{div}_{i_{k+1}} C_{g}^{\zeta, i_{1} \ldots i_{k+1}} \\
& \quad+\sum_{\zeta \in Z_{b, \mathfrak{p}}} a_{\zeta} X \operatorname{div}_{i_{1}} C_{g}^{\zeta, i_{1}}+\sum_{\zeta \in Z_{O K}} a_{\zeta} X \operatorname{div}_{i_{1}} \ldots X \operatorname{div}_{i_{a}} C_{g}^{\zeta, i_{1} \ldots i_{a}}+\sum_{j \in} a_{j} C^{j}
\end{aligned}
$$

The tensor fields indexed in $\bar{Z}_{a, \mathfrak{p}}^{3}, Z_{b, \mathfrak{p}}$ on the right-hand side are generic linear combinations in those forms (the first with rank $k+1$ ). The tensor fields indexed in $Z_{O K}$ are generic linear combinations as allowed in the right-hand side of our Step 2.

Iteratively repeating this step we are reduced to proving Step 2 when $Z_{a, \mathfrak{p}}=\varnothing$. In that case we then assume that the tensor fields indexed in $Z_{b, \mathfrak{p}}$ have minimum rank $k$ (and the corresponding index set is $Z_{b, \mathfrak{p}}^{k}$ ) and we show that we can write

$$
\begin{array}{r}
\sum_{\zeta \in Z_{b, \mathfrak{p}}^{k}} a_{\zeta} X \operatorname{div}_{i_{1}} \ldots X \operatorname{div}_{i_{k}} C_{g}^{\zeta, i_{1} \ldots i_{k}}=\sum_{\zeta \in Z_{b, \mathfrak{p}}^{k+1}} a_{\zeta} X \operatorname{div}_{i_{1}} \ldots X \operatorname{div}_{i_{k+1}} C_{g}^{\zeta, i_{1} \ldots i_{k+1}} \\
+\sum_{\zeta \in Z_{O K}} a_{\zeta} X \operatorname{div}_{i_{1}} \ldots X \operatorname{div}_{i_{a}} C_{g}^{\zeta, i_{1} \ldots i_{a}}+\sum_{j \in} a_{j} C^{j},
\end{array}
$$

(with the same conventions as in the above equation).

If we can prove (2-105) and (2-107) we will have shown our Step 2.

Proof of (2-105), (2-106), (2-107). We start with a small remark: If the chosen factor is of the form $S_{*} \nabla^{(v)} R_{i j k l}$, our assumption implies a more convenient equation: Consider the tensor fields $C_{g}^{\zeta, i_{1} \ldots i_{a}}, \zeta \in \bar{Z}_{a, \mathfrak{p}} \cup Z_{b, \mathfrak{p}}$; we denote by $\tilde{C}_{g}^{\zeta, i_{1} \ldots i_{a}}$ the tensor fields that arise from $C_{g}^{\zeta, i_{1} \ldots i_{a}}$ by replacing the expression

$$
\nabla_{r_{1} \ldots r_{v}}^{(v)} R_{i j k l} \nabla^{i} \tilde{\phi}_{1} \nabla^{k} Y \quad\left(\text { or } \nabla_{r_{1} \ldots r_{v}}^{(v)} R_{i j k l} \nabla^{i} \tilde{\phi}_{1} \nabla^{k} \chi_{2}\right)
$$

by a factor $\nabla_{r_{1} \ldots r_{v} j l}^{(v+2)} \Omega_{p+1}$. We then derive

$$
\begin{aligned}
\sum_{\zeta \in \bar{Z}_{a} \cup Z_{b}} a_{\zeta} X_{*} & \operatorname{div}_{i_{1}} \ldots X_{*} \operatorname{div}_{i_{a}} \\
\times & \tilde{C}_{g}^{\zeta, i_{1} \ldots i_{a}}\left(\Omega_{1}, \ldots, \Omega_{p+1}, \phi_{2}, \ldots, \phi_{u},\left(\chi_{1}\right), \psi_{1}, \ldots, \psi_{\tau}\right) \\
& =\sum_{j \in J} a_{j} C_{g}^{j}\left(\Omega_{1}, \ldots, \Omega_{p+1}, \phi_{2}, \ldots, \phi_{u},\left(\chi_{1}\right), \psi_{1}, \ldots, \psi_{\tau}\right) .
\end{aligned}
$$

Now we can derive our claims. 
Proof of (2-106). We split the index set $Z_{\bar{Z}_{a, \mathfrak{p}}^{2}}$ according to the two factors that contain the two free indices and we show our claim for each of those tensor fields separately. The proof goes as follows: We pick out the sublinear combination in our hypothesis (or in (2-108)) where the factors $\nabla \psi_{h}, \nabla \psi_{h^{\prime}}\left(\right.$ or $\left.\nabla \psi_{h}, \nabla \chi_{2}\right)$ contract against the same factor. Clearly, this sublinear combination, $X_{g}$, vanishes separately. We then formally erase the factor $\nabla \psi_{h}$. Then, we apply the inductive assumption of our general claim to the resulting equation (the minimum rank of the tensor fields will be 1), and (in case our assumption is (2-108) we also apply an operation $\mathrm{Op}^{-1}$ which replaces the factor $\nabla_{r_{1} \ldots r_{y}}^{(y)} \Omega_{p+1}$ by

$$
S_{*} \nabla_{r_{1} \ldots r_{y-2}}^{(y-2)} R_{i r_{y-1} k r_{y}} \nabla^{i} \tilde{\phi}_{1} \nabla^{k} Y\left(\nabla^{k} \chi_{1}\right)
$$

This is our desired conclusion.

Proof of (2-105), (2-107). Now we show (2-105) for the subset $Z_{a, \mathfrak{p}}^{k, \alpha}$ (which indexes the $k$-tensor fields for which the free indices $i_{1}, \ldots, i_{k}$ belong to a chosen subset of the factors $\nabla \psi_{1}, \ldots, \nabla \psi_{\tau},\left(\nabla \chi_{1}\right)$ (hence the label $\alpha$ designates the chosen subset). To prove this equation, we pick out the sublinear combination in (2-108) where the factors $\nabla \psi_{2}, \ldots, \nabla \psi_{\tau},\left(\nabla \chi_{1}\right)$ (indexed in $\left.\alpha\right)$ contract against the same factor as $\nabla \psi_{1}$. Then we apply the eraser to these factors and the indices they contract against. This is our desired conclusion. To show (2-107), we only have to treat the factors $\nabla \psi_{h}$ as factors $\nabla \phi_{h}$. The claim then follows by applying Corollary 1 in [A 2010] and making the factors $\nabla v$ into $X$ divs. $^{70}$

Proof of the base case $(v+b=2)$ of the general claim. We first prove our claim when our hypothesis is (2-67) (as opposed to (2-66)).

Proof of the base case under the hypothesis (2-67). We observe that the weight $-K$ in our assumption must satisfy $K \geq 2 \tau+8$ if $v>0$ and $K \geq 2 \tau+6$ if $v=0$.

First consider the case where we have the strict inequalities $K>2 \tau+8$ if $v>0$ and $K>2 \tau+6$ if $v=0$. In that case our first claim of the base case can be proven straightforwardly, by picking out a removable index in each $C_{g}^{\zeta, i_{a}}, \zeta \in Z_{a}$ and treating it as an $X_{*} \operatorname{div}$ (which can be done when we only have two real factors). Thus, in this setting we only have to show our second claims (2-70), (2-71).

In this setting, by using the "manual" constructions as in [A 2011], we can construct explicit tensor fields which satisfy all the assumptions of our claim in the

${ }^{70}$ Observe that by virtue of the factor $\nabla \psi_{1}$, we must have at least one nonsimple factor $S_{*} \nabla^{(v)} R_{i j k l}$ or $\nabla^{(B)} \Omega_{h}$ in (2-108)-hence (2-108) does not fall under any of the "forbidden cases" of Corollary 1 in [A 2010], by inspection. 
base case (each with rank $\geq 2$ ), so that

$$
\begin{aligned}
& X_{+} \operatorname{div}_{i_{1}} \sum_{\zeta \in Z_{a}^{\prime} \cup Z_{b}} a_{\zeta} C_{g}^{\zeta, i_{1}}\left(\Omega_{1}, \ldots, \Omega_{b}, \phi_{1}, \ldots, \phi_{v},\left[\chi_{1}, \chi_{2}\right], \psi_{1}, \ldots, \psi_{\tau}\right) \\
& =\sum_{q \in Q} a_{q} X_{+} \operatorname{div}_{i_{1}} C_{g}^{q, i_{q}}\left(\Omega_{1}, \ldots, \Omega_{b}, \phi_{1}, \ldots, \phi_{v},\left[\chi_{1}, \chi_{2}\right], \psi_{1}, \ldots, \psi_{\tau}\right) \\
& \quad+\sum_{p \in P} a_{p} X_{+} \operatorname{div}_{i_{1}} \ldots X_{+} \operatorname{div}_{i_{c+1}} \\
& \quad+C_{g}^{p, i_{1} \ldots i_{c+1}}\left(\Omega_{1}, \ldots, \Omega_{b}, \phi_{1}, \ldots, \phi_{v},\left[\chi_{1}, \chi_{2}\right], \psi_{1}, \ldots, \psi_{\tau}\right) \\
& \quad+\sum_{j \in J} a_{j} C_{g}^{j} .
\end{aligned}
$$

Here the tensor field $C_{g}^{p, i_{1} \ldots i_{c+1}}$ will be in one of three forms:

- If $v=2$ then each $C_{g}^{p, i_{1} \ldots i_{c+1}}$ will be in the form

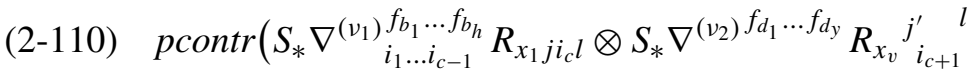

$$
\left.\otimes\left[\nabla^{j} \chi_{1} \otimes \nabla_{j^{\prime}} \chi_{2}\right] \otimes \nabla_{f_{1}} \psi_{1} \cdots \otimes \nabla_{f_{\tau}} \psi_{\tau} \otimes \nabla^{x_{1}} \tilde{\phi}_{1} \otimes \nabla^{x_{2}} \tilde{\phi}_{2}\right),
$$

where $\left\{b_{1}, \ldots, b_{h}, d_{1}, \ldots, d_{y}\right\}=\{1, \ldots, \tau\}$.

- If $v=1$ then $\sum_{p \in P} \cdots=0$ (this can be arranged because of the two antisymmetric indices $_{k}, l$ in the one factor $S_{*} \nabla^{(v)} R_{i j k l}$ ).

- If $v=0$ then each $C_{g}^{p, i_{1} \ldots i_{c+1}}$ will be in the form

$$
\begin{aligned}
& \operatorname{pcontr}\left(\nabla^{\left(A_{1}\right)} \underset{{ }_{1} \ldots f_{b_{1}} \ldots f_{b_{h}}}{f_{i_{c}}} \Omega_{1} \otimes \nabla^{\left(A_{2}\right)} f_{d_{1} \ldots f_{d_{y}} j^{\prime} i_{c+1}} \Omega_{2}\right. \\
& \left.\quad \otimes\left[\nabla^{j} \chi_{1} \otimes \nabla_{j^{\prime}} \chi_{2}\right] \otimes \nabla_{f_{1}} \psi_{1} \cdots \otimes \nabla_{f_{\tau}} \psi_{\tau} \otimes \nabla^{x_{1}} \tilde{\phi}_{1} \otimes \nabla^{x_{2}} \tilde{\phi}_{2}\right),
\end{aligned}
$$

where $\left\{b_{1}, \ldots, b_{h}, d_{1}, \ldots, d_{y}\right\}=\{1, \ldots, \tau\}$.

Then, picking out the sublinear combination in (2-110), (2-111) with factors $\nabla \psi_{1}, \ldots, \nabla \psi_{\tau}, \nabla \chi_{1}, \nabla \chi_{2}$, we derive that $\sum_{p \in P} \cdots=0$. This is precisely our desired conclusion in this case.

Now, the case where we have the equalities in our lemma hypothesis, $K=2 \tau+8$ if $v>0$ and $K=2 \tau+6$ if $v=0$. In this case we note that in our hypothesis $Z_{b}=\varnothing$ if $v \neq 1$, while $Z_{a}=\bar{Z}_{a}=\varnothing$ if $v=1$.

Then, if $v \neq 1$, by the "manual" constructions as in [A 2011], it follows that we can construct tensor fields (as required in the claim of our "general claim"), so 
that:

$$
\begin{aligned}
& \sum_{\zeta \in Z_{a}} a_{\zeta} C_{g}^{\zeta, i_{1}}\left(\Omega_{1}, \ldots, \Omega_{b}, \phi_{1}, \ldots, \phi_{v},\left[\chi_{1}, \chi_{2}\right], \psi_{1}, \ldots, \psi_{\tau}\right) \nabla_{i_{1}} v \\
& \times X_{*} \operatorname{div}_{i_{2}} a_{\zeta} C_{g}^{\zeta, i_{1} i_{2}}\left(\Omega_{1}, \ldots, \Omega_{b}, \phi_{1}, \ldots, \phi_{v},\left[\chi_{1}, \chi_{2}\right], \psi_{1}, \ldots, \psi_{\tau}\right) \nabla_{i_{1}} v \\
& =a_{*} C_{g}^{*, i_{1}}\left(\Omega_{1}, \ldots, \Omega_{b}, \phi_{1}, \ldots, \phi_{v},\left[\chi_{1}, \chi_{2}\right], \psi_{1}, \ldots, \psi_{\tau}\right) \nabla_{i_{1}} v \\
& \quad+\sum_{j \in J} a_{j} C_{g}^{j, i_{1}}\left(\Omega_{1}, \ldots, \Omega_{b}, \phi_{1}, \ldots, \phi_{v},\left[\chi_{1}, \chi_{2}\right], \psi_{1}, \ldots, \psi_{\tau}\right) \nabla_{i_{1}} v
\end{aligned}
$$

where the tensor field $C_{g}^{*, i_{1}}$ is in the form

$$
\begin{aligned}
& \operatorname{pcontr}\left(S_{*} \nabla^{\left(v_{1}\right) f_{1} \ldots f_{\tau-1}} R_{x_{1}}^{f_{\tau}}{ }_{k l} \otimes R_{x_{2}}^{j^{\prime} k l}\right. \\
& \left.\quad \otimes\left[\nabla_{i_{1}} \chi_{1} \otimes \nabla_{j^{\prime}} \chi_{2}\right] \otimes \nabla_{f_{1}} \psi_{1} \cdots \otimes \nabla_{f_{\tau}} \psi_{\tau} \otimes \nabla^{x_{1}} \tilde{\phi}_{1} \otimes \nabla^{x_{2}} \tilde{\phi}_{2}\right),
\end{aligned}
$$

if $v=2$, and in the form

$$
\begin{aligned}
& \operatorname{pcontr}\left(\nabla^{(\tau+1) f_{1} \ldots f_{\tau}} \Omega_{1} \otimes \nabla^{j^{\prime} s} \Omega_{2}\right. \\
& \left.\quad \otimes\left[\nabla_{i_{1}} \chi_{1} \otimes \nabla_{j^{\prime}} \chi_{2}\right] \otimes \nabla_{f_{1}} \psi_{1} \cdots \otimes \nabla_{f_{\tau}} \psi_{\tau} \otimes \nabla^{x_{1}} \tilde{\phi}_{1} \otimes \nabla^{x_{2}} \tilde{\phi}_{2}\right),
\end{aligned}
$$

if $v=0$.

Thus, we are reduced to the case where $Z_{a}$ only consists of the vector field (2-113) or (2-114), and all other tensor fields in our lemma hypothesis have rank $\geq 2$ (we have denoted their index set by $Z_{a}^{\prime}$ ). We then show that we can write

$$
\begin{aligned}
& X_{+} \operatorname{div}_{i_{1}} \sum_{\zeta \in Z_{a}^{\prime}} a_{\zeta} C_{g}^{\zeta, i_{1}}\left(\Omega_{1}, \ldots, \Omega_{b}, \phi_{1}, \ldots, \phi_{v},\left[\chi_{1}, \chi_{2}\right], \psi_{1}, \ldots, \psi_{\tau}\right) \\
& =\sum_{q \in Q} a_{q} X_{+} \operatorname{div}_{i_{1}} C_{g}^{q, i_{1}}\left(\Omega_{1}, \ldots, \Omega_{b}, \phi_{1}, \ldots, \phi_{v},\left[\chi_{1}, \chi_{2}\right], \psi_{1}, \ldots, \psi_{\tau}\right) \\
& \quad+\sum_{p \in P} a_{p} X_{+} \operatorname{div}_{i_{1}} \ldots X_{+} \operatorname{div}_{i_{c+1}} \\
& \quad \times C_{g}^{p, i_{1} \ldots i_{c+1}}\left(\Omega_{1}, \ldots, \Omega_{b}, \phi_{1}, \ldots, \phi_{v},\left[\chi_{1}, \chi_{2}\right], \psi_{1}, \ldots, \psi_{\tau}\right) \\
& \quad+\sum_{j \in J} a_{j} C_{g}^{j},
\end{aligned}
$$

where the tensor fields indexed in $P$ here each have rank $\geq 2$ and are all in one of the forms

(2-116) $\operatorname{pcontr}\left(S_{*} \nabla^{\left(v_{1}\right) f_{1} \ldots f_{\tau-1}} R_{x_{1}}^{f_{\tau}}{ }_{i_{k} l} \otimes S_{*} R_{x_{v}}^{j^{\prime} k l}\right.$

$$
\left.\otimes\left[\nabla_{i_{1}} \chi_{1} \otimes \nabla_{j^{\prime}} \chi_{2}\right] \otimes \nabla_{y_{1}} \psi_{1} \cdots \otimes \nabla_{y_{\tau}} \psi_{\tau} \otimes \nabla^{x_{1}} \tilde{\phi}_{1} \otimes \nabla^{x_{2}} \tilde{\phi}_{2}\right),
$$

or

(2-117) $\operatorname{pcontr}\left(\nabla^{\left(\nu_{1}\right)} f_{1} \ldots f_{\tau} \Omega_{1} \otimes \nabla^{j^{\prime} s} \Omega_{2} \otimes\left[\nabla_{i_{1}} \chi_{1} \otimes \nabla_{j^{\prime}} \chi_{2}\right] \otimes \nabla_{y_{1}} \psi_{1} \cdots \otimes \nabla_{y_{\tau}} \psi_{\tau}\right)$, 
where each of the indices ${ }^{f_{h}}$ contracts against one of the indices $y_{q}$. The indices $y_{q}$ that do not contract against an index ${ }^{f_{h}}$ are free indices.

Then, replacing the above into our lemma hypothesis (and making all the $\nabla v \mathrm{~s}$ into $X_{+}$divs), we derive that $a_{p}=0$ for every $p \in P$ and $a_{*}=0$. This concludes the proof of the base case when $v+b=2, v \neq 1$. In the case $v=1$ we show our claim by just observing that we can write

$$
\begin{aligned}
X_{+} \operatorname{div}_{i_{1}} \sum_{\zeta \in Z_{b}} a_{\zeta} C_{g}^{\zeta, i_{1}}\left(\Omega_{1}, \ldots, \Omega_{b}, \phi_{1},\left[\chi_{1}, \chi_{2}\right], \psi_{1}, \ldots, \psi_{\tau}\right) & \\
= & \sum_{q \in Q} a_{q} X_{+} \operatorname{div}_{i_{1}} C_{g}^{q, i_{1}}\left(\Omega_{1}, \ldots, \Omega_{b}, \phi_{1},\left[\chi_{1}, \chi_{2}\right], \psi_{1}, \ldots, \psi_{\tau}\right) \\
& \quad+\sum_{j \in J} a_{j} C_{g}^{j}\left(\Omega_{1}, \ldots, \Omega_{b}, \phi_{1},\left[\chi_{1}, \chi_{2}\right], \psi_{1}, \ldots, \psi_{\tau}\right)
\end{aligned}
$$

this concludes the proof of the base case, when the tensor fields in our lemma hypothesis are in the form (2-67).

Now, we consider the setting where our hypothesis is (2-66). We again observe that if $v=0$ then the weight $-K$ in our hypothesis must satisfy $K \geq 2 \tau+4$. If $v>0$ it must satisfy $K \geq 2 \tau+6$. We then again first consider the case where we have the strict inequalities in the hypothesis of our general claim.

In this case (where we have the strict inequalities $K>2 \tau+4$ if $v=0$ and $K>2 \tau+6$ if $v \neq 0$ ) our first claim follows straightforwardly (as above, we just pick out one removable index in each $C_{g}^{\zeta, i_{1}}, \zeta \in Z_{a}$ and treat it as an $X_{*}$ div). To show the second claim we proceed much as before:

We can "manually" construct tensor fields in order to write

$$
\begin{aligned}
& X_{+} \operatorname{div}_{i_{1}} \sum_{\zeta \in Z_{a}^{\prime} \cup Z_{b}} a_{\zeta} C_{g}^{\zeta, i_{1}}\left(\Omega_{1}, \ldots, \Omega_{b}, \phi_{1}, \ldots, \phi_{v}, Y, \psi_{1}, \ldots, \psi_{\tau}\right) \\
& =\sum_{q \in Q} a_{q} X_{+} \operatorname{div}_{i_{1}} C_{g}^{q, i_{q}}\left(\Omega_{1}, \ldots, \Omega_{b}, \phi_{1}, \ldots, \phi_{v},\left[\chi_{1}, \chi_{2}\right], \psi_{1}, \ldots, \psi_{\tau}\right) \\
& \quad+\sum_{p \in P} a_{p} X_{+} \operatorname{div}_{i_{1}} \ldots X_{+} \operatorname{div}_{i_{c+1}} \\
& \quad \times C_{g}^{p, i_{1} \ldots i_{c+1}}\left(\Omega_{1}, \ldots, \Omega_{b}, \phi_{1}, \ldots, \phi_{v},\left[\chi_{1}, \chi_{2}\right], \psi_{1}, \ldots, \psi_{\tau}\right) \\
& \quad+\sum_{j \in J} a_{j} C_{g}^{j} .
\end{aligned}
$$

Here the tensor field $C_{g}^{p, i_{1} \ldots i_{c+1}}$ will be in one of three forms: 
- If $v=2$ then each $C_{g}^{p, i_{1} \ldots i_{c+1}}$ will be

$$
\begin{aligned}
\operatorname{pcontr}\left(S_{*} \nabla^{\left(v_{1}\right)}{ }_{{ }_{1} \ldots i_{c-1}}^{f_{b_{1}} \ldots f_{b_{h}}} R_{x_{1}}{ }_{{ }_{b_{h+1}}}^{f_{i_{c} l}} \otimes S_{*} \nabla^{\left(v_{2}\right)}{ }_{f_{d_{1}} \ldots f_{d_{y}}} R_{x_{v}}{ }^{{ }_{f_{d_{y+1}}}{ }_{i_{c+1}}{ }^{l}}\right. \\
\left.\otimes \nabla_{f_{\tau+1}} Y \otimes \nabla_{f_{1}} \psi_{1} \cdots \otimes \nabla_{f_{\tau}} \psi_{\tau} \otimes \nabla^{x_{1}} \tilde{\phi}_{1} \otimes \nabla^{x_{2}} \tilde{\phi}_{2}\right),
\end{aligned}
$$

where $\left\{b_{1}, \ldots, b_{h+1}, d_{1}, \ldots, d_{y+1}\right\}=\{1, \ldots, \tau+1\}$.

- If $v=1$ then $\sum_{p \in P} \cdots=0$ (this is because of the two antisymmetric indices ${ }_{k}, l$ in the one factor $\left.S_{*} \nabla^{(v)} R_{i j k l}\right)$.

- If $v=0$ then each $C_{g}^{p, i_{1} \ldots i_{c+1}}$ will be in the form

$$
\begin{aligned}
\operatorname{pcontr}\left(\nabla^{\left(A_{1}\right)}{ }_{i_{1} \ldots i_{c-1} i_{c}}^{f_{b_{1}} \ldots f_{b_{h}}} \Omega_{1} \otimes \nabla^{\left(A_{2}\right)} f_{d_{1} \ldots f_{d_{y}} i_{c+1}} \Omega_{2}\right. & \\
& \left.\otimes \nabla_{f_{\tau+1}} Y \otimes \nabla_{f_{1}} \psi_{1} \cdots \otimes \nabla_{f_{\tau}} \psi_{\tau}\right),
\end{aligned}
$$

where $\left\{b_{1}, \ldots, b_{h}, d_{1}, \ldots, d_{y}\right\}=\{1, \ldots, \tau+1\}$.

Then, picking out the sublinear combination in (2-120), (2-121) with factors $\nabla \psi_{1}, \ldots, \nabla \psi_{\tau}, \nabla Y$ we derive that $\sum_{p \in P} \cdots=0$. This is precisely our desired conclusion in this case.

Finally, we prove our claim when we have the equalities $K=2 \tau+4$ if $v<2$ and $K=2 \tau+6$ if $v=2$ ) in the hypothesis of our general claim.

In this case by "manually" constructing $X_{+}$divs so that we can write

$$
\begin{aligned}
& \sum_{\zeta \in Z_{a}^{\prime} \cup Z_{b} \cup \bar{Z}_{a}} a_{\zeta} X_{+} \operatorname{div}_{i_{1}} \ldots X_{+} \operatorname{div}_{i_{a}} C_{g}^{\zeta, i_{1} \ldots i_{a}}\left(\Omega_{1}, \ldots \Omega_{b}, Y, \psi_{1}, \ldots, \psi_{\tau}\right) \\
& =\sum_{q \in Q} a_{q} X_{+} \operatorname{div}_{i_{1}} \ldots X_{+} \operatorname{div}_{i_{a}} C_{g}^{q, i_{1} \ldots i_{a}}\left(\Omega_{1}, \ldots \Omega_{b}, Y, \psi_{1}, \ldots, \psi_{\tau}\right) \\
& \quad+\sum_{p \in P} a_{p} X_{+} \operatorname{div}_{i_{1}} \ldots X_{+} \operatorname{div}_{i_{a}} C_{g}^{p, i_{1} \ldots i_{a}}\left(\Omega_{1}, \ldots \Omega_{b}, Y, \psi_{1}, \ldots, \psi_{\tau}\right) \\
& \quad+\sum_{j \in J} a_{j} C_{g}^{j}\left(\Omega_{1}, \ldots \Omega_{b}, Y, \psi_{1}, \ldots, \psi_{\tau}\right) .
\end{aligned}
$$

Here the tensor fields indexed in $P$ are in specific forms:

- If $v=0$ then they will either be in the form

(2-123) pcontr $\left(\nabla_{i_{*}} Y \otimes \nabla^{(A)} f_{x_{1} \cdots f_{x_{a}} s} \Omega_{1} \otimes \nabla^{(B)}{ }_{s}^{f_{x_{a+1}} \cdots f_{x_{\tau}}} \Omega_{2} \otimes \nabla_{f_{1}} \psi_{1} \otimes \cdots \otimes \nabla_{f_{\tau}} \phi_{\tau}\right)$, where $\left\{x_{1}, \ldots, x_{\tau}\right\}=\{1, \ldots, \tau\}$, or in the form (2-124) $\operatorname{pcontr}\left(\nabla_{q} Y \otimes \nabla^{(A)}{ }_{i_{*}}^{f_{x_{1}} \ldots f_{x_{a}}} \Omega_{1} \otimes \nabla^{(B)} f_{x_{a+1} \ldots f_{x_{\tau}} q} \Omega_{2} \otimes \nabla_{f_{1}} \psi_{1} \otimes \cdots \otimes \nabla_{f_{\tau}} \phi_{\tau}\right)$, where $\left\{x_{1}, \ldots, x_{\tau}\right\}=\{1, \ldots, \tau\}$. 
- If $v=2$ they will be in the form

$$
\begin{array}{r}
\operatorname{pcontr}\left(\nabla_{i_{*}} Y \otimes \nabla^{(A) f_{x_{1}} \ldots f_{x_{a-1}}} S_{*} R^{i f_{x_{a}} k l} \otimes \nabla^{(B) f_{x_{a+1}} \ldots f_{x_{\tau-1}}} R^{i^{\prime} f_{x_{\tau}}} k l\right. \\
\left.\otimes \nabla_{f_{1}} \psi_{1} \otimes \cdots \otimes \nabla_{f_{\tau}} \phi_{\tau} \nabla_{i} \tilde{\phi}_{1} \otimes \nabla_{i^{\prime}} \tilde{\phi}_{2}\right),
\end{array}
$$

where $\left\{x_{1}, \ldots, x_{\tau}\right\}=\{1, \ldots, \tau\}$, or in the form

$$
\begin{array}{r}
\operatorname{pcontr}\left(\nabla_{q} Y \otimes \nabla^{(A) f_{x_{1}} \ldots f_{x_{a-1}}} S_{*} R^{i f_{x_{a}} q l} \otimes \nabla^{(B) f_{x_{a+1}} \cdots f_{x_{\tau}-1}} R^{i^{\prime} f_{x_{\tau}}} i_{* l} l\right. \\
\left.\otimes \nabla_{y_{1}} \psi_{1} \otimes \cdots \otimes \nabla_{y_{\tau}} \phi_{\tau} \nabla_{i} \tilde{\phi}_{1} \otimes \nabla_{i^{\prime}} \tilde{\phi}_{2}\right) .
\end{array}
$$

- If $v=1$, Equation (2-122) will hold with $P=\varnothing$.

Then, picking out the sublinear combination in (2-122) which consists of terms with a factor $\nabla Y$ and replacing into our hypothesis, we derive that the coefficient of each of the tensor fields indexed in $P$ must be zero. This completes the proof of our claim.

\section{E. Proof of Lemmas 2.2 and 2.4.}

Proof of Lemma 2.2. The first claim follows immediately, since each tensor field has a removable index (thus each tensor field separately can be written as an $X_{*}$ div).

The proof of the second claim essentially follows the "manual" construction of divergences, as in [A 2011]. By "manually" constructing explicit divergences out of each $C_{g}^{h, i_{1} \ldots i_{\alpha}}\left(\Omega_{1}, \ldots, \Omega_{p}, \phi_{1}, \ldots, \phi_{u}\right), h \in H_{2}$, we derive that we can write

$$
\begin{aligned}
\sum_{h \in H_{2}} & a_{h} X \operatorname{div}_{i_{1}} \ldots X \operatorname{div}_{i_{\alpha}} C_{g}^{h, i_{1} \ldots i_{\alpha}}\left(\Omega_{1}, \ldots, \Omega_{p}, Y, \phi_{1}, \ldots, \phi_{u}\right) \\
= & (\text { Const })_{1} X \operatorname{div}_{i_{1}} \ldots X \operatorname{div}_{i_{\xi}} C_{g}^{1, i_{1} \ldots i_{\xi}}\left(\Omega_{1}, \ldots, \Omega_{p}, Y, \phi_{1}, \ldots, \phi_{u}\right) \\
& +(\text { Const })_{2} X \operatorname{div}_{i_{1}} \ldots X \operatorname{div}_{i_{\zeta}} C_{g}^{2, i_{1} \ldots i_{\zeta}}\left(\Omega_{1}, \ldots, \Omega_{p}, Y, \phi_{1}, \ldots, \phi_{u}\right) \\
& +\sum_{q \in Q} a_{q} X \operatorname{div}_{i_{1}} \ldots X \operatorname{div}_{i_{\alpha}} C_{g}^{q, i_{1} \ldots i_{\alpha}}\left(\Omega_{1}, \ldots, \Omega_{p}, Y, \phi_{1}, \ldots, \phi_{u}\right) \\
& +\sum_{j \in J} a_{j} C_{g}^{j}\left(\Omega_{1}, \ldots, \Omega_{p}, Y, \phi_{1}, \ldots, \phi_{u}\right)
\end{aligned}
$$

where the tensor fields indexed in $Q$ are as required by our lemma hypothesis, while the tensor fields $C^{1}, C^{2}$ are explicit tensor fields which we will write out below (their precise form depends on the values $\left.p, \sigma_{1}, \sigma_{2}\right){ }^{71}$

We will then show that in (2-127) we will have $(\text { Const })_{1}=(\text { Const })_{2}=0$. That will complete the proof of Lemma 2.2. We distinguish cases based on the value of $p$ : Either $p=2$ or $p=1$ or $p=0$.

${ }^{71}$ In some cases there will be no tensor fields $C^{1}, C^{2}$ (in which case we will just say that in $(2-127)$ we have $\left.(\text { Const })_{1}=0,(\text { Const })_{2}=0\right)$. 
The case $p=2$. With no loss of generality we assume that $\nabla^{(A)} \Omega_{1}$ contracts against $\nabla \phi_{1}, \ldots, \nabla \phi_{x}$ and that $\nabla^{(B)} \Omega_{2}$ contracts against $\nabla \phi_{x+1}, \ldots, \nabla \phi_{x+t}$; we may also assume without loss of generality that $x \leq t$. By manually constructing divergences, it follows that we can derive (2-127), where each of the tensor fields $C^{1}, C^{2}$ will be in the forms, respectively,

$$
\operatorname{pcontr}\left(\nabla_{i_{*}} Y \otimes \nabla_{v_{1} \ldots v_{x} i_{1} \ldots i_{\gamma}}^{(A)} \Omega_{1} \otimes \nabla_{y_{1} \ldots y_{t} i_{\gamma+1} \ldots i_{\gamma+\delta}}^{(B)} \Omega_{2} \otimes \nabla^{v_{1}} \phi_{1} \otimes \cdots \otimes \nabla^{y_{t}} \phi_{u}\right) \text {, }
$$

where if $t \geq 2$ then $\delta=0$, otherwise $t+\delta=2$; or

$$
\begin{array}{r}
\operatorname{pcontr}\left(\nabla_{q} Y \otimes \nabla^{q} \nabla_{v_{1} \ldots v_{x} i_{1} \ldots i_{\gamma}}^{(A)} \Omega_{1} \otimes \nabla_{y_{1} \ldots y_{t} i_{\gamma+1} \ldots i_{\gamma+\delta}}^{(B)} \Omega_{2}\right. \\
\left.\otimes \nabla^{v_{1}} \phi_{1} \otimes \cdots \otimes \nabla^{y_{t}} \phi_{u}\right),
\end{array}
$$

where if $t \geq 2$ then $\delta=0$, otherwise $t+\delta=2$.

The case $p=1$. We "manually" construct divergences to derive (2-127), where if $\sigma_{1}=1$ then there are no tensor fields $C^{1}, C^{2}$ (and hence (2-127) is our desired conclusion); if $\sigma_{1}=0, \sigma_{2}=1$ then there is only the tensor field $C^{1}$ in (2-127) and it is in the form

$$
\begin{aligned}
\operatorname{pcontr}\left(\nabla^{q} Y \otimes S_{*} \nabla_{v_{2} \ldots v_{x} i_{1} \ldots i_{\gamma}}^{(v)} R_{i i_{\gamma+1} i_{\gamma+2} q}\right. & \otimes \nabla_{y_{1} \ldots y_{t} i_{\gamma+1} \ldots i_{\gamma+\delta}}^{(B)} \Omega_{2} \\
& \left.\otimes \nabla^{i} \tilde{\phi}_{1} \otimes \nabla^{v_{1}} \phi_{2} \otimes \cdots \otimes \nabla^{y_{t}} \phi_{u}\right),
\end{aligned}
$$

where if $t \geq 2$ then $\delta=0$, otherwise $\delta=2-t$.

The case $p=0$. We have three subcases: First $\sigma_{2}=2$, second $\sigma_{2}=1$ and $\sigma_{1}=1$, and third $\sigma_{1}=2$.

In the case $\sigma_{2}=2$, the tensor fields $C^{1}, C^{2}$ must be in the forms, respectively,

(2-131) $p$ contr $\left(\nabla_{i_{*}} Y \otimes S_{*} \nabla_{v_{2} \ldots v_{x} i_{1} \ldots i_{\gamma}}^{(v)} R_{i i_{\gamma+1} i_{\gamma+2} l} \otimes S_{*} \nabla_{y_{1} \ldots y_{t}}^{(t-1)} R_{i^{\prime} i_{\gamma+3} i_{\gamma+4}}{ }^{l}\right.$

$$
\left.\otimes \nabla^{i} \tilde{\phi}_{1} \otimes \nabla^{i^{\prime}} \tilde{\phi}_{2} \otimes \nabla^{v_{1}} \phi_{3} \otimes \cdots \otimes \nabla^{y_{t}} \phi_{u}\right)
$$

or

$$
\begin{aligned}
& \operatorname{pcontr}\left(\nabla^{q} Y \otimes S_{*} \nabla_{q v_{2} \ldots v_{x} i_{1} \ldots i_{\gamma}}^{(v)}\right. R_{i i_{\gamma+1} i_{\gamma+2} l} \otimes S_{*} \nabla_{y_{1} \ldots y_{t}}^{(t-1)} R_{i^{\prime} i_{\gamma+3} i_{\gamma+4}}{ }^{l} \\
&\left.\otimes \nabla^{i} \tilde{\phi}_{1} \otimes \nabla^{i^{\prime}} \tilde{\phi}_{2} \otimes \nabla^{v_{1}} \phi_{3} \otimes \cdots \otimes \nabla^{y_{t}} \phi_{u}\right),
\end{aligned}
$$

(if $x=t=0$ then the tensor field $C^{1}$ above will not be present).

In the case $\sigma_{1}=2$, the tensor fields $C^{1}, C^{2}$ must be in one of the two forms

$$
\begin{array}{r}
\operatorname{pcontr}\left(\nabla_{i_{*}} Y \otimes \nabla_{v_{1} \ldots v_{x} i_{1} \ldots i_{\gamma}}^{\left(m_{1}\right)} R_{i i_{\gamma+1} i_{\gamma+2} l} \otimes \nabla_{y_{1} \ldots y_{t}}^{(t-1)} R_{i_{\gamma+3} i_{\gamma+4}}^{i}{ }^{l}\right. \\
\left.\otimes \nabla^{v_{1}} \phi_{1} \otimes \cdots \otimes \nabla^{y_{t}} \phi_{u}\right),
\end{array}
$$


or

$$
\begin{aligned}
\operatorname{pcontr}\left(\nabla_{q} Y \otimes \nabla^{q} \nabla_{v_{1} \ldots v_{x} i_{1} \ldots i_{\gamma}}^{\left(m_{1}\right)} R_{i i_{\gamma+1} i_{\gamma+2} l} \otimes \nabla_{y_{1} \ldots y_{t}}^{(t-1)} R_{i_{\gamma+3} i_{\gamma+4}}^{i}\right. & \\
& \left.\otimes \nabla^{v_{1}} \phi_{1} \otimes \cdots \otimes \nabla^{y_{t}} \phi_{u}\right) .
\end{aligned}
$$

In the case $\sigma_{1}=1$ and $\sigma_{2}=1$, there will be only one tensor field $C^{1}$, in the form

$$
\begin{aligned}
\operatorname{pcontr}\left(\nabla^{q} Y \otimes S_{*} \nabla_{v_{1} \ldots v_{x} i_{1} \ldots i_{\gamma}}^{\left(m_{1}\right)} R_{i i_{\gamma+1} i_{\gamma+2} l} \otimes \nabla_{y_{1} \ldots y_{t}}^{(t-1)} R_{q i_{\gamma+3} i_{\gamma+4}}{ }^{l}\right. & \\
& \left.\otimes \nabla^{i} \tilde{\phi}_{1} \otimes \nabla^{v_{1}} \phi_{2} \otimes \cdots \otimes \nabla^{y_{t}} \phi_{u}\right) .
\end{aligned}
$$

We then derive that $(\text { Const })_{1}=(\text { Const })_{2}=0$ as in [A 2011] (by picking out the sublinear combination in (2-127) that consists of complete contractions with a factor $\nabla Y$ - differentiated only once).

Proof of Lemma 2.4. We again "manually" construct explicit $X$ div to write

$$
\begin{aligned}
& \sum_{h \in H_{2}} a_{h} X \operatorname{div}_{i_{1}} \ldots X \operatorname{div}_{i_{\alpha}} C_{g}^{h, i_{1} \ldots i_{\alpha}}\left(\Omega_{1}, \ldots, \Omega_{p}, Y, \phi_{1}, \ldots, \phi_{u}\right) \\
& =(\text { Const })_{1} X \operatorname{div}_{i_{1}} \ldots X \operatorname{div}_{i_{\xi}} C_{g}^{1, i_{1} \ldots i_{\xi}}\left(\Omega_{1}, \ldots, \Omega_{p}, Y, \phi_{1}, \ldots, \phi_{u}\right) \\
& \quad+(C o n s t)_{2} X \operatorname{div}_{i_{1}} \ldots X \operatorname{div}_{i_{\zeta}} C_{g}^{2, i_{1} \ldots i_{\zeta}}\left(\Omega_{1}, \ldots, \Omega_{p}, Y, \phi_{1}, \ldots, \phi_{u}\right) \\
& \quad+\sum_{q \in Q} a_{q} X \operatorname{div}_{i_{1}} \ldots X \operatorname{div}_{i_{\alpha}} C_{g}^{q, i_{1} \ldots i_{\alpha}}\left(\Omega_{1}, \ldots, \Omega_{p}, Y, \phi_{1}, \ldots, \phi_{u}\right) \\
& \quad+\sum_{j \in J} a_{j} C_{g}^{j}\left(\Omega_{1}, \ldots, \Omega_{p}, Y, \phi_{1}, \ldots, \phi_{u}\right)
\end{aligned}
$$

where the tensor fields indexed in $Q$ are as required by our lemma hypothesis, while the tensor fields $C^{1}, C^{2}$ are explicit tensor fields which we will write out below (they depend on the values $p, \sigma_{1}, \sigma_{2}$ ). In some cases there will be no tensor fields $C^{1}, C^{2}$ (in which case we will just say that in (2-127) we have $(\text { Const })_{1}=0$, $\left.(\text { Const })_{2}=0\right)$.

The case $p=2$. With no loss of generality we assume that $\nabla^{(A)} \Omega_{1}$ contracts against $\nabla \phi_{1}, \ldots, \nabla \phi_{x}$ and that $\nabla^{(B)} \Omega_{2}$ contracts against $\nabla \phi_{x+1}, \ldots, \nabla_{\phi_{x+t}}$; we may also assume without loss of generality that $x \leq t$. By manual construction of divergences, it follows that we can derive (2-127), where there is only the tensor field $C^{1}$ and it is in the form

$$
\begin{aligned}
\operatorname{pcontr}\left(\nabla_{\left[i_{*}\right.} \chi_{1} \otimes \nabla^{q]} \chi_{2} \otimes \nabla_{v_{1} \ldots v_{x} i_{1} \ldots i_{\gamma}}^{(A)} \Omega_{1} \otimes \nabla_{q y_{1} \ldots y_{t} i_{\gamma+1} \ldots i_{\gamma+\delta}}^{(B)} \Omega_{2}\right. & \\
& \left.\otimes \nabla^{v_{1}} \phi_{1} \otimes \cdots \otimes \nabla^{y_{t}} \phi_{u}\right),
\end{aligned}
$$

where if $t \geq 1$ then $\delta=0$, otherwise $\delta=1$.

The case $p=1$. We "manually" construct divergences to derive (2-136), where if $\sigma_{1}=1$ then there are no tensor fields $C^{1}, C^{2}$ in the right-hand side of (2-136) 
(and this is our desired conclusion); and if $\sigma_{1}=0$ and $\sigma_{2}=1$ then there is only the tensor field $C^{1}$ in the right-hand side of (2-136) and it is of the form

$$
\begin{array}{r}
\operatorname{pcontr}\left(\nabla_{\left[i_{*}\right.} \omega_{1} \otimes \nabla^{q]} \omega_{2} \otimes S_{*} \nabla_{v_{2} \ldots v_{x} i_{1} \ldots i_{\gamma}}^{(v)} R_{i i_{\gamma+1} i_{\gamma+2} q} \otimes \nabla_{y_{1} \ldots y_{t} i_{\gamma+1} \ldots i_{\gamma+\delta}}^{(B)} \Omega_{2}\right. \\
\left.\otimes \nabla^{i} \tilde{\phi}_{1} \otimes \nabla^{v_{1}} \phi_{2} \otimes \cdots \otimes \nabla^{y_{t}} \phi_{u}\right),
\end{array}
$$

where if $t \geq 2$ then $\delta=0$, otherwise $\delta=2-t$.

The case $p=0$. We have three subcases: First $\sigma_{2}=2$, second $\sigma_{2}=1$ and $\sigma_{1}=1$, and third $\sigma_{1}=2$.

In the case $\sigma_{2}=2$, the tensor fields $C^{1}, C^{2}$ in the right-hand side of (2-127) will be in the two forms, respectively,

$$
\begin{aligned}
\operatorname{pcontr} & \left(\nabla_{\left[i_{*}\right.} \omega_{1} \otimes \nabla^{q]} \omega_{2} \otimes S_{*} \nabla_{q v_{2} \ldots v_{x} i_{1} \ldots i_{\gamma}}^{(v)} R_{i i_{\gamma+1} i_{\gamma+2} l}\right. \\
& \left.\otimes S_{*} \nabla_{y_{1} \ldots y_{t}}^{(t-1)} R_{i^{\prime} i_{\gamma+3} i_{\gamma+4}}{ }^{l} \otimes \nabla^{i} \tilde{\phi}_{1} \otimes \nabla^{i^{\prime}} \tilde{\phi}_{2} \otimes \nabla^{v_{1}} \phi_{3} \otimes \cdots \otimes \nabla^{y_{t}} \phi_{u}\right),
\end{aligned}
$$

or

$$
\begin{aligned}
\operatorname{pcontr} & \left(\nabla_{[p} \omega_{1} \otimes \nabla^{q]} \omega_{2} \otimes S_{*} \nabla_{q v_{2} \ldots v_{x} i_{1} \ldots i_{\gamma}}^{(v)} R_{i i_{\gamma+1} i_{\gamma+2} p}\right. \\
& \left.\otimes S_{*} \nabla_{y_{1} \ldots y_{t}}^{(t-1)} R_{i^{\prime} i_{\gamma+3} i_{\gamma+4} q} \otimes \nabla^{i} \tilde{\phi}_{1} \otimes \nabla^{i^{\prime}} \tilde{\phi}_{2} \otimes \nabla^{v_{1}} \phi_{3} \otimes \cdots \otimes \nabla^{y_{t}} \phi_{u}\right) .
\end{aligned}
$$

In the case $\sigma_{1}=2$, the tensor fields $C^{1}, C^{2}$ will be the forms, respectively,

(2-141) $p \operatorname{contr}\left(\nabla_{\left[i_{*}\right.} \omega_{1} \otimes \nabla^{q]} \otimes \nabla_{v_{1} \ldots v_{x} i_{1} \ldots i_{\gamma}}^{\left(m_{1}\right)} R_{i i_{\gamma+1} i_{\gamma+2} l} \otimes \nabla_{q y_{1} \ldots y_{t}}^{(t-1)} R_{i_{\gamma+3} i_{\gamma+4}}{ }^{l}\right.$

$$
\left.\otimes \nabla^{v_{1}} \phi_{1} \otimes \cdots \otimes \nabla^{y_{t}} \phi_{u}\right)
$$

or

(2-142) $\operatorname{pcontr}\left(\nabla_{[p} \omega_{1} \otimes \nabla^{q]} \omega_{2} \otimes \nabla_{v_{1} \ldots v_{x} i_{1} \ldots i_{\gamma}}^{\left(m_{1}\right)} R_{i i_{\gamma+1} i_{\gamma+2} p} \otimes \nabla_{y_{1} \ldots y_{t}}^{(t-1)} R_{i_{\gamma+3} i_{\gamma+4} q}^{i_{1}}\right.$

$$
\left.\otimes \nabla^{v_{1}} \phi_{1} \otimes \cdots \otimes \nabla^{y_{t}} \phi_{u}\right)
$$

if at least one of the two factors $\nabla^{(m)} R_{i j k l}$ contracts against a factor $\nabla \phi_{h}$, otherwise we can prove (2-136) with no tensor fields $C^{1}, C^{2}$ on the right-hand side.

In the case $\sigma_{1}=1, \sigma_{2}=1$, the tensor fields $C^{1}, C^{2}$ must be in the forms, respectively,

(2-143) $\operatorname{pcontr}\left(\nabla_{\left[i_{*}\right.} \omega_{1} \otimes \nabla^{q]} \omega_{2} \otimes S_{*} \nabla_{v_{1} \ldots v_{x} i_{1} \ldots i_{\gamma}}^{(v)} R_{i i_{\gamma+1} i_{\gamma+2} l} \otimes \nabla_{y_{1} \ldots y_{t}}^{(t-1)} R_{q i_{\gamma+3} i_{\gamma+4}}{ }^{l}\right.$

$$
\left.\otimes \nabla^{i} \tilde{\phi}_{1} \otimes \nabla^{v_{1}} \phi_{2} \otimes \cdots \otimes \nabla^{y_{t}} \phi_{u}\right)
$$

or

(2-144) $\operatorname{pcontr}\left(\nabla_{[p} \omega_{1} \otimes \nabla^{q]} \omega_{2} \otimes S_{*} \nabla_{v_{1} \ldots v_{x} i_{1} \ldots i_{\gamma}}^{\left(m_{1}\right)} R_{i i_{\gamma+1} i_{\gamma+2} l} \otimes \nabla_{y_{1} \ldots y_{t}}^{(t-1)} R_{p q i_{\gamma+3}}{ }^{l}\right.$ $\left.\otimes \nabla^{i} \tilde{\phi}_{1} \otimes \nabla^{v_{1}} \phi_{2} \otimes \cdots \otimes \nabla^{y_{t}} \phi_{u}\right)$. 
We then derive that $(\text { Const })_{1}=(\text { Const })_{2}=0$ by picking out the sublinear combination in (2-136) that consists of complete contractions with two factors $\nabla Y, \nabla \omega_{2}$ - each factor differentiated only once.

\section{The proof of Proposition 1.1 in the special cases}

3A. The direct proof of Proposition 1.1 (in Case II) in the special cases. We now prove Proposition 1.1 directly in the special subcases of Case II. We recall that the settings of the special subcases of Proposition 1.1 in Case II are as follows: In Subcase IIA for each $\mu$-tensor field (in (1-7)) of maximal refined double character, $C_{g}^{l, i_{1} \ldots i_{\mu}}$ there is a unique factor in the form $T=\nabla^{(m)} R_{i j k l}$ for which two internal indices are free, and each derivative index is either free or contracting against a factor $\nabla \phi_{h}$. For Subcase IIB there is a unique factor in the form $T=\nabla^{(m)} R_{i j k l}$ for which one internal index is free, and each derivative index is either free or contracting against a factor $\nabla \phi_{h}$. In both Subcases IIA, IIB there is at least one free derivative index in the factor $T$.

Moreover, in both Subcases IIA and IIB, all other factors in one of the forms $\nabla^{(m)} R_{i j k l}, S_{*} \nabla^{(v)} R_{i j k l}, \nabla^{(p)} \Omega_{h}$ in $C_{g}^{l, i_{1} \ldots i_{\mu}}$ are either in the form $S_{*} R_{i j k l}$ or $\nabla^{(2)} \Omega_{h}$, or they are in the form $\nabla^{(m)} R_{i j k l}$, where all the $m$ derivative indices contract against factors $\nabla \phi_{h}{ }^{72}$

In order to prove Proposition 1.1 directly in the special subcases of Subcases IIA, IIB we will rely on a new lemma. It deals with two different settings, which we will label Setting A and Setting B below.

In Setting A, we let

$$
\sum_{l \in \bar{L}} a_{l} C_{g}^{l, i_{1} \ldots i_{\mu}}\left(\Omega_{1}, \ldots, \Omega_{p}, \phi_{1}, \ldots, \phi_{u}\right)
$$

stand for a linear combination of $\mu$-tensor fields with one factor $\nabla^{(m)} R_{i j k l}$ containing $\alpha \geq 2$ free indices, distributed according to the pattern $\nabla_{(\text {free }) \ldots(\text { free })}^{(m)} R_{(\text {free }) j(\text { free }) l}$, and all other factors being in one of the forms $R_{i j k l}, S_{*} R_{i j k l}, \nabla^{(2)} \Omega_{h}$. (In particular they have no removable indices.)

In Setting B we let

$$
\sum_{l \in \bar{L}} a_{l} C_{g}^{l, i_{1} \ldots i_{\mu}}\left(\Omega_{1}, \ldots, \Omega_{p}, \phi_{1}, \ldots, \phi_{u}\right)
$$

stand for a linear combination of $\mu$-tensor fields with one factor $\nabla^{(m)} R_{i j k l}$ containing $\alpha \geq 2$ free indices, distributed according to the pattern $\nabla_{(\text {free }) \ldots(\text { free })}^{(m)} R_{(\text {free }) j(\text { free }) l}$, and all but one of the other factors being in one of the forms $R_{i j k l}, S_{*} R_{i j k l}, \nabla^{(2)} \Omega_{h}$;

${ }^{72}$ For the rest of this subsection, we will slightly abuse notation and not write out the derivative indices that contract against factors $\nabla \phi_{h}$ - we will thus refer to factors $R_{i j k l}$, setting $m=0$. 
one of the other factors (which we label $T^{\prime}$ ) will be in the form $\nabla R_{i j k l}, S_{*} \nabla R_{i j k l}$, $\nabla^{(3)} \Omega_{h}$. We will call this other factor "the factor with the extra derivative". Moreover, in Setting B we impose the additional restriction that if both the indices ${ }_{j}, l$ in the factor $\nabla_{(\text {free }) \ldots(\text { free })}^{(m)} R_{(\text {free }) j(\text { free }) l}$ contract against the same other factor $T^{\prime}$, then either $T^{\prime}$ is not the factor with the extra derivative, or if it is, then $T^{\prime}$ is in the form $\nabla_{s} R_{a b c d}$, and furthermore the indices ${ }_{j}, l$ contract against the indices ${ }_{b},{ }_{c}$ and we assume that the indices ${ }_{s}, a, c$ are symmetrized over. ${ }^{73}$

Lemma 3.1. Let $\sum_{l \in \bar{L}} a_{l} C_{g}^{l, i_{1} \ldots i_{\mu}}$ be a linear combination of $\mu$-tensor fields as described above. We assume the following special case of (1-7),

$$
\begin{aligned}
& \sum_{l \in \bar{L} \cup L^{\prime}} a_{l} X \operatorname{div}_{i_{1}} \ldots X \operatorname{div}_{i_{\mu}} C_{g}^{l, i_{1} \ldots i_{\mu}}\left(\Omega_{1}, \ldots, \Omega_{p}, \phi_{1}, \ldots, \phi_{u}\right) \\
& \quad+\sum_{h \in H} a_{h} X \operatorname{div}_{i_{1}} \ldots X \operatorname{div}_{i_{\beta}} C_{g}^{h, i_{1} \ldots i_{\beta}}\left(\Omega_{1}, \ldots, \Omega_{p}, \phi_{1}, \ldots, \phi_{u}\right) \\
& =\sum_{j \in J} a_{j} C_{g}^{j}\left(\Omega_{1}, \ldots, \Omega_{p}, \phi_{1}, \ldots, \phi_{u}\right),
\end{aligned}
$$

holds; here, in both Cases $A$ and $B$ the terms indexed in $\bar{L}$ will be as described above; the $\mu$-tensor fields indexed in $L^{\prime}$ will have fewer than $\alpha$ free indices in any given factor of the form $\nabla^{(m)} R_{i j k l}$. The tensor fields indexed in $H$ each have rank $>\mu$ and also each of them has fewer than $\alpha$ free indices in any given factor of the form $\nabla^{(m)} R_{i j k l}$. Finally, the terms indexed in $J$ are simply subsequent to $\vec{\kappa}_{\text {simp. }}$.

We claim that

$$
\sum_{l \in \bar{L}} a_{l} C_{g}^{l, i_{1} \ldots i_{\mu}} \nabla_{i_{1}} v \ldots \nabla_{i_{\mu}} v=0 .
$$

We will prove this lemma shortly. Let us now prove that the above lemma directly implies Proposition 1.1 in the special Subcases IIA (directly) and IIB (after some manipulation).

Lemma 3.1 implies Proposition 1.1 in the special subcases of Case II. We first start with Subcase IIA: Consider the sublinear combination of $\mu$-tensor fields of maximal refined double character in (1-7). Denote their index set by $L_{\operatorname{Max}} \subset L$. Recall that since we are considering the subcase where (1-7) falls under the special case of Proposition 1.1 in Case IIA, it follows that for each $C_{g}^{l, i_{1} \ldots i_{\mu}}$ there is a unique factor in the form $\nabla^{(m)} R_{i j k l}$ for which two internal indices are free, and each derivative index is either free or contracting against a factor $\nabla \phi_{h}$; denote by $M+2$ the number of free indices in that factor. ${ }^{74}$

${ }^{73}$ To put it in other words, in that case the two factors $T, T^{\prime}$ contract according to the pattern $\nabla_{(\text {free }) \ldots(\text { free })}^{(m)} R_{(\text {free }) j(\text { free }) l} \nabla_{(s} R_{a}{ }^{j k}{ }_{d)}$, where the indices $s, a, d$ are symmetrized over.

${ }^{74}$ So we set $\alpha=M+2$. 
By weight considerations (since we are in a special subcase of Proposition 1.1 in Case IIA), any tensor field of rank $>\mu$ in (1-7) must have strictly fewer than $M+2$ free indices in any given factor $\nabla^{(m)} R_{i j k l}$. Therefore in Subcase IA, (1-7) is of the form (3-1), with $L_{\operatorname{Max}} \subset \bar{L}$. Therefore, we apply Lemma 3.1 to (1-7) and pick out the sublinear combination of terms with a refined double character $\operatorname{Doub}\left(\vec{L}^{z}\right), z \in Z_{\text {Max }}^{\prime}{ }^{75}$ we thus obtain a new true equation, since (3-2) holds formally, and the double character is invariant under the formal permutations of indices that make (3-2) formally zero. This proves our claim in Subcase IIA.

Now we deal with Subcase IIB: We consider the $\mu$-tensor fields of maximal refined double character in (1-7). By definition (since we now fall under a special case), they will each have a factor in the form $\nabla_{(\text {free }) \ldots(f r e e)}^{(m)} R_{(f r e e) j k l}$, with a total of $M+1>1$ free indices. ${ }^{76}$ Each of the other factors will be in the form $R_{i j k l}$ or be simple factors in the form $S_{*} R_{i j k l}$, or in the form $\nabla^{(2)} \Omega_{h}$.

We denote by $\bar{L} \subset L$ the index set of $\mu$-tensor fields with $M+1$ free indices in a factor $\nabla^{(m)} R_{i j k l}$. It follows by weight considerations that the factor in question will be unique for each $C_{g}^{l, i_{1} \ldots i_{\mu}}, l \in \bar{L}$. We then start out with some explicit manipulation of the terms indexed in $\bar{L}$ :

We will prove that there exists a linear combination of $(\mu+1)$-tensor fields, $\sum_{h \in H} a_{h} C_{g}^{h, i_{1} \ldots i_{\mu+1}}$, as allowed in the statement of Proposition 1.1, so that

$$
\begin{aligned}
\sum_{l \in \bar{L}} a_{l} C_{g}^{l, i_{1} \ldots i_{\mu}} \nabla_{i_{1}} v \ldots \nabla_{i_{\mu}} v=\sum_{h \in H} a_{h} X \operatorname{div}_{i_{\mu+1}} C_{g}^{h, i_{1} \ldots i_{\mu+1}} \nabla_{i_{1}} v \ldots \nabla_{i_{\mu}} v \\
+\sum_{l \in \bar{L}_{\text {new }}} a_{l} C_{g}^{l, i_{1} \ldots i_{\mu}} \nabla_{i_{1}} v \ldots \nabla_{i_{\mu}} v \sum_{j \in J} a_{j} C_{g}^{l, i_{1} \ldots i_{\mu}} \nabla_{i_{1}} v \ldots \nabla_{i_{\mu}} v .
\end{aligned}
$$

Here the $\mu$-tensor fields indexed in $\bar{L}_{n e w}$ have a factor $T=\nabla_{(\text {free }) . .(\text { free })}^{(M-1)} R_{(\text {free }) j(\text { free }) l}$, and one other factor $T^{\prime}$ has an extra derivative (meaning that $T^{\prime}$ is either in the form $\nabla R_{i j k l}$ or $S_{*} \nabla R_{i j k l}$, or $\left.\nabla^{(3)} \Omega_{h}\right)$. Moreover if both indices ${ }_{j}, l$ in $T$ contract against indices ${ }^{j},{ }^{l}$ in the same factor $T^{\prime \prime}$ and at least one of ${ }^{j},{ }^{l}$ is removable, then $T^{\prime} \neq T^{\prime \prime}$. Clearly, (3-3) in conjunction with Lemma 3.1 implies Proposition 1.1 in the "special cases" of Case II. So, matters are reduced to showing (3-3) (and then deriving Lemma 3.1).

Proof of (3-3). Apply the second Bianchi identity to the factor $T$ to move one of the derivative free indices into the position ${ }_{k}, l$ in the factor $\nabla_{(\text {free }) \ldots(\text { free })}^{(M-1)} R_{(\text {free }) j(\text { free }) l}$. Thus, we derive that modulo terms of length $\geq \sigma+u+1$,

$$
C_{g}^{l, i_{1} \ldots i_{\mu}}=-C_{g}^{l, 1, i_{1} \ldots, i_{\mu}}+C_{g}^{l, 2, i_{1} \ldots, i_{\mu}},
$$

\footnotetext{
${ }^{75}$ Recall that $\vec{L}^{z}, z \in Z_{\text {Max }}^{\prime}$, is the collection of maximal refined double characters that Proposition 1.1 deals with.

${ }^{76}$ So, we set $\alpha=M+1$.
} 
where the partial contractions $C_{g}^{l, 1, i_{1} \ldots i_{\mu}}$ and $C_{g}^{l, 2, i_{1} \ldots i_{\mu}}$ have the factor $T$ replaced by a factor in the form

$$
\nabla_{k(\text { free }) \ldots(\text { free })}^{(m)} R_{(\text {free }) j(\text { free }) l} \quad \text { and } \quad \nabla_{l(\text { free }) \ldots(\text { free })}^{(m)} R_{(\text {free }) j k(\text { free })},
$$

respectively. We then erase the indices ${ }_{k},{ }_{l}$ in these two factors (thus creating new tensor fields $C_{g}^{l, 1, i_{1} \ldots, i_{\mu} i_{\mu+1}}$ and $C_{g}^{\left.l, 2, i_{1} \ldots, i_{\mu} i_{\mu+1}\right)}$ by creating a free index $i_{\mu+1}$, and subtract the $X \operatorname{div}_{i_{\mu+1}}[\ldots]$ of the corresponding $(\mu+1)$-tensor field. We then derive

$$
C_{g}^{l, 1, i_{1} \ldots, i_{\mu}}=X \operatorname{div}_{i_{\mu+1}} C_{g}^{l, 1, i_{1} \ldots i_{\mu+1}}+\sum_{l \in L_{\text {new }}} C_{g}^{l, i_{1} \ldots i_{\mu}},
$$

where all the fields indexed in $L_{\text {new }}$ satisfy the required property of Lemma 3.1, except for that one could have both indices ${ }_{j},{ }_{l}$ in the factor $\nabla_{(\text {free }) . . .(\text { free })}^{(M-1)} R_{(\text {free }) j(\text { free }) l}$ contracting against indices ${ }^{j},{ }^{l}$ in a factor $T^{\prime}$ which has an additional derivative index. If $C_{g}^{l, i_{1} \ldots i_{\mu}}, l \in L_{\text {new }}$, is not in the form allowed in the claim of Lemma 3.1, then (after possibly applying the second Bianchi identity and possibly introducing simply subsequent complete contractions) we may arrange that one of the indices ${ }^{j}, l$ is a derivative index.

In that case we construct another $(\mu+1)$-tensor field by erasing the derivative index ${ }^{j}$ or ${ }^{l}$ and making the index ${ }_{j}$ or ${ }_{l}$ in a free index $i_{\mu+1}$. Then, subtracting the corresponding $X \operatorname{div}_{i_{\mu+1}}$ of this new $(\mu+1)$-tensor field, we derive our claim.

Therefore, matters are reduced to proving Lemma 3.1.

Proof of Lemma 3.1. Let us start with some notational conventions.

Recall the first variation law of the curvature tensor under variations by a symmetric 2-tensor by $v_{i j}$ : For any complete or partial contraction $T\left(g_{i j}\right)$ (which is a function of the metric $g_{i j}$ ), we define

$$
\text { Image }_{v_{i j}}^{1}=\left.\frac{d}{d t}\right|_{t=0}\left[T\left(g_{i j}+t v_{i j}\right)\right] .
$$

(We write Image $e_{v_{i j}}^{1}[\ldots]$ or Image $e_{v_{a b}}^{1}[\ldots]$ below to stress that we are varying by a 2-tensor, rather than just by a scalar.)

We consider the equation Image $e_{v_{i j}}^{1}\left[L_{g}\right]=0$ (which corresponds to the first metric variation of our lemma hypothesis (that is, of (1-7)). This equation holds modulo complete contractions with at least $\sigma+u+1$ factors.

Thus, we derive a new local equation,

$$
\begin{aligned}
& \sum_{l \in L_{\mu}} a_{l} X \operatorname{div}_{i_{1}} \ldots X \operatorname{div}_{i_{\mu}} \operatorname{Image}_{v_{a b}}^{1}\left[C_{g}^{l, i_{1} \ldots i_{\mu}}\right] \\
& \quad+\sum_{l \in L \backslash L_{\mu}} a_{l} X \operatorname{div}_{i_{1}} \ldots X \operatorname{div}_{i_{a}} \operatorname{Image}_{v_{a b}}^{1}\left[C_{g}^{l, i_{1} \ldots i_{a}}\right]=\sum_{j \in J} a_{j} \operatorname{Image}_{v_{a b}}^{1}\left[C_{g}^{j}\right],
\end{aligned}
$$

which holds modulo terms of length $\geq \sigma+u+1$. 
Now, we wish to pass from the local equation above to an integral equation, and then to apply the silly divergence formula from [A 2009] to that integral equation (thus deriving a new local equation).

In order to do this, we start by introducing some more notation: Let us write out

$$
\operatorname{Image}_{v_{a b}}^{1}\left[C_{g}^{l, i_{1} \ldots i_{\mu}}\right]=\sum_{t \in T^{l}} a_{t} C_{g}^{t, i_{1} \ldots i_{a}},
$$

where each $C_{g}^{t, i_{1} \ldots i_{a}}$ is in the form

$$
\begin{aligned}
\operatorname{pcontr}\left(\nabla_{r_{1} \ldots r_{A+2}}^{(A+2)} v_{a b} \otimes \nabla^{\left(m_{1}\right)} R_{i j k l} \otimes \cdots \otimes \nabla^{\left(m_{\sigma-1}\right)} R\right. & \\
& \left.\otimes \nabla^{\left(b_{1}\right)} \Omega_{1} \otimes \cdots \otimes \nabla^{\left(b_{p}\right)} \Omega_{p} \otimes \nabla \phi_{1} \otimes \cdots \otimes \nabla \phi_{u}\right) .
\end{aligned}
$$

For our next technical tool we introduce some notation: For each tensor field $C_{g}^{l, i_{1} \ldots i_{a}}$ in the form above, we denote by $C_{g}^{l}$ the complete contraction that arises by hitting each factor $T_{i}(i=1,2,3)$ by $m$ derivative indices $\nabla^{u_{1} \ldots u_{m}}$, where ${ }_{u_{1}}, \ldots, u_{m}$ are the free indices that belong to $T_{i}$ in $C_{g}^{l, i_{1} \ldots i_{a}}$ (thus we obtain a factor with $m$ internal contraction, each involving a derivative index). Notice there is a one-toone correspondence between the tensor fields and the complete contractions we are constructing. We can then easily observe that there are two linear combinations

$$
\begin{gathered}
\sum_{r \in R_{1}} a_{r} C_{g}^{r}\left(\Omega_{1}, \ldots \Omega_{p}, \phi_{1}, \ldots, \phi_{u}\right), \\
\sum_{r \in R_{2}} a_{r} C_{g}^{r}\left(\Omega_{1}, \ldots \Omega_{p}, \phi_{1}, \ldots, \phi_{u}\right),
\end{gathered}
$$

where each $C_{g}^{r}, r \in R_{1}$ has at least $\sigma+u+1$ factors, while each $C_{g}^{r}, r \in R_{2}$ has $\sigma+u$ factors but at least one factor $\nabla^{(p)} \phi_{h} \neq \Delta \phi_{h}$ with $p \geq 2$, so that for any compact orientable $(M, g)$,

$$
\int_{M} \sum_{l \in L} a_{l} \sum_{t \in T^{l}} a_{t} C_{g}^{t, *}\left(v_{a b}\right)+\sum_{r \in R_{1}} a_{r} C_{g}^{r}\left(v_{a b}\right)+\sum_{r \in R_{2}} a_{r} C_{g}^{r}\left(v_{a b}\right) d V_{g}=0
$$

(denote the integrand of the above by $Z_{g}\left(v_{a b}\right)$ ). Here again each $C_{g}^{j}$ has $\sigma+u$ factors and all factors $\nabla \phi_{h}$ have only one derivative but its simple character is subsequent to $\vec{\kappa}$. We call this technique (of going from the local equation (3-5) to the integral equation (3-7)) the " inverse integration by parts".

Now, we derive a "silly divergence formula" from the above by performing integrations by parts with respect to the factor $\nabla^{(B)} v_{a b}$ (until we are left with a factor $v_{a b}$-without derivatives). This produces a new local equation which we denote by silly $\left[Z_{g}\left(v_{a b}\right)\right]=0$. We will be using this equation in our derivation of Lemma 3.1. 
Now, for each $C_{g}^{l, i_{1} \ldots i_{\mu}}, l \in \bar{L}$, we consider the factor

$$
T=\nabla_{(\text {free }) \ldots(\text { free })}^{(M)} R_{(\text {free }) j(\text { free }) l}
$$

with the $M+2$ free indices. We define $T^{j}$ to be the factor in $C_{g}^{l, i_{1} \ldots i_{\mu}}$ that contracts against the index ${ }_{j}$ in $T$ and by $T^{l}$ to be the factor in $C_{g}^{l, i_{1} \ldots i_{\mu}}$ that contracts against the index ${ }_{l}$ in $T$. We define $\bar{L}_{\text {same }} \subset \bar{L}$ to be the index set of tensor fields for which $T^{j}=T^{l}$; we define $\bar{L}_{\text {not.same }} \subset \bar{L}$ to be the index set of tensor fields for which $T^{j} \neq T^{l}$. We will then prove (3-2) separately for the two sublinear combinations indexed in $\bar{L}_{\text {same }}, \bar{L}_{\text {not.same }}$.

Proof of (3-2) for the index set $\bar{L}_{\text {same. }}$. We first prove our claim for $\sigma>3$ and then note how to prove it when $\sigma=3$.

Consider silly $\left[L_{g}\left(\Omega_{1}, \ldots, \Omega_{p}, \phi_{1}, \ldots, \phi_{u}, v_{a b}\right)\right]=0$. Pick out the sublinear combination silly ${ }_{+}\left[L_{g}\left(\Omega_{1}, \ldots, \Omega_{p}, \phi_{1}, \ldots, \phi_{u}, v_{a b}\right)\right]=0$ with $\mu-M-2$ internal contractions, and with the indices in the factor $v_{a b}$ contracting against a factor $T^{\prime}$ which either has no extra derivative indices, or if it does, then the contraction is according to the pattern $v^{a b} \otimes \nabla_{s} R_{a j b l}$; we also require that the two factors $T^{\prime \prime}$, $T^{\prime \prime \prime}$ with an extra $M+2$ extra derivatives each. This sublinear combination must vanish separately, hence we derive

$$
\text { silly }_{+}\left[Z_{g}\left(\Omega_{1}, \ldots, \Omega_{p}, \phi_{1}, \ldots, \phi_{u}, v_{a b}\right)\right]=0 .
$$

We also observe that this sublinear combination can only arise (in the process of passing from the equation $L_{g}=0$ to deriving silly ${ }_{+}\left[Z_{g}\left(v_{a b}\right)\right]=0$ ) by replacing the factor $\nabla_{(\text {free }) \ldots(\text { free })}^{(M)} R_{(\text {free }) j(\text { free }) l}$ by $\nabla_{(\text {free }) \ldots(\text { free })}^{(M)} v_{j l}$ and then (in the inverse integration by parts) replacing all $\mu$ free indices by internal contractions, ${ }^{77}$ and finally integrating by parts the $M+2$ pairs of derivative indices $\left(\nabla^{a}, \nabla_{a}\right)$ and forcing all upper indices hit a factor $T^{\prime \prime} \neq T^{\prime}$ and the lower indices to hit a factor $T^{\prime \prime \prime} \neq T^{\prime}$, $T^{\prime \prime \prime} \neq T^{\prime \prime} .^{78}$

Thus, we can prove our claim by starting from (3-8) and applying $\mathrm{Sub}_{v} \mu-M-2$ times, ${ }^{79}$ just applying the eraser to the extra $M+2$ pairs of contracting derivatives, ${ }^{80}$ and then replacing the factor $v_{a b}$ by $\nabla_{r_{1} \ldots r_{M}}^{(M)} R_{i a j b} \nabla^{r_{1}} v \ldots \nabla^{r_{M}} v \nabla^{a} v \nabla^{b} v$. Finally we just divide by the combinatorial constant $\left(\begin{array}{c}\sigma-3 \\ 2\end{array}\right)$.

Let us now consider the case $\sigma=3$ : In this case the terms of maximal refined double character can only arise in Subcase IIA, ${ }^{81}$ and can only be in one of the

${ }^{77}$ Thus the factor $\nabla\left(\begin{array}{c}M) \\ (\text { free }) \ldots(\text { free })\end{array} v_{j l}\right.$ gets replaced by $\Delta^{M+2} v_{i j}$.

${ }^{78}$ The fact that $\sigma>3$ ensures the existence of two such factors.

${ }^{79}$ See the Appendix in [A 2012] for this operation, and just set $\omega=v$.

${ }^{80}$ This can be done by repeating the proof of the "Eraser" lemma in the Appendix in [A 2012].

${ }^{81}$ This follows by the symmetry of the indices ${ }_{s}, a, d$ in any factor $\nabla_{s} R_{a b c d}$ as discussed above. 
forms

$$
\begin{aligned}
& \nabla_{(\text {free }) \ldots(\text { free })}^{(M)} R_{(\text {free }) j(\text { free }) l} \otimes R^{i j k l} \otimes \nabla_{i k}^{(2)} \Omega_{1}, \\
& \nabla_{(\text {free }) \ldots(\text { free })}^{(\text {free }) j(\text { free }) l} \otimes R^{i j k l} \otimes R_{(\text {free }) i(f r e e) k} .
\end{aligned}
$$

Thus, in that case we define silly ${ }_{+}\left[Z_{g}\left(v_{a b}\right)\right]$ to stand for the terms

$$
\begin{aligned}
& v_{j l} \otimes \nabla_{t_{1} \ldots t_{M+2}}^{(M+2)} R^{i j k l} \otimes \nabla_{t_{1} \ldots t_{M+4} i k}^{(M+4)} \Omega_{1}, \\
& v_{j l} \otimes \nabla_{t_{1} \ldots t_{M+2}}^{(M+2)} R^{i j k l}\left(\nabla^{(M+2)}\right)^{t_{1} \ldots t_{M+2}} \otimes R_{(\text {free }) i(f r e e) k},
\end{aligned}
$$

respectively, and then repeat the argument above.

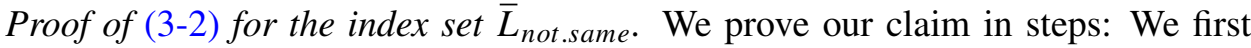
denote by $\bar{L}_{\text {not.same }}^{* *} \subset \bar{L}_{\text {not.same }}$ the index set of tensor fields in $\bar{L}_{\text {not.same }}$ for which both indices ${ }_{j}, l$ in the factor $T=\nabla_{(\text {free }) \ldots(\text { free })}^{(M)} R_{(\text {free }) j(\text { free }) l}$ contract against special indices in factors $T^{j}, T^{l}$ of the form $S_{*} R_{i j k l}$. We will first prove that

$$
\sum_{l \in \bar{L}_{\text {not.same }}^{* *}} a_{l} C_{g}^{l, i_{1} \ldots i_{\mu}} \nabla_{i_{1}} v \ldots \nabla_{i_{\mu}} v=\sum_{l \in L^{\prime}} a_{l} C_{g}^{l, i_{1} \ldots i_{\mu}} \nabla_{i_{1}} v \ldots \nabla_{i_{\mu}} v
$$

Here the terms in the right-hand side have all the features of the terms in $\bar{L}_{\text {not.same }}$, but in addition at most one of the indices in the factor $T=\nabla_{(\text {free }) . .(\text { free })}^{(M)} R_{(\text {free }) j(f r e e) l}$ contract against a special index in a factor of the form $S_{*} R_{i j k l}$. Thus, if we can prove (3-9), we are reduced to proving our claim under the additional assumption that $\bar{L}_{\text {not.same }}^{* *}=\varnothing$.

For our next claim, we denote by $\bar{L}_{\text {not.same }}^{*} \subset \bar{L}_{\text {not.same }}$ the index set of tensor fields in $\bar{L}_{\text {not.same }}$ for which one of the indices ${ }_{j}, l$ in the factor

$$
T=\nabla_{(\text {free }) \ldots(\text { free })}^{(M)} R_{(\text {free }) j(\text { free }) l}
$$

contracts against a special index in factors $T^{j}, T^{l}$ of the form $S_{*} R_{i j k l}$.

We will then prove that

$$
\sum_{l \in \bar{L}_{\text {not.same }}^{*}} a_{l} C_{g}^{l, i_{1} \ldots i_{\mu}} \nabla_{i_{1}} v \ldots \nabla_{i_{\mu}} v=\sum_{l \in L^{\prime \prime}} a_{l} C_{g}^{l, i_{1} \ldots i_{\mu}} \nabla_{i_{1}} v \ldots \nabla_{i_{\mu}} v
$$

Here the terms in the right-hand side have all the features of the terms in $\bar{L}_{\text {not.same }}$, but in addition none of the indices in the factor $T=\nabla_{(\text {free }) \ldots(\text { free })}^{(M)} R_{(\text {free }) j(f r e e) l}$ contract against a special index in a factors of the form $S_{*} R_{i j k l}$. Thus, if we can prove (3-9), we are reduced to proving our claim under the further assumption that for each $C_{g}^{l, i_{1} \ldots i_{\mu}}, l \in \bar{L}$, the two indices ${ }_{j}, l$ in the factor $T=\nabla_{(\text {free }) \ldots(\text { free })}^{(M)} R_{(\text {free }) j(\text { free }) l}$ contract against two different factors and none of the indices ${ }^{j},{ }^{l}$ are special indices in a factor of the form $S_{*} R_{i j k l}$. 
In our third step, we prove (3-2) under this additional assumption. We will indicate in the end how this proof can be easily modified to derive the first two steps.

For each $l \in \bar{L}_{\text {not.same }}$, let us denote by $\operatorname{link}(l)$ the number of particular contractions between the factors $T^{j}, T^{l}$ in the tensor fields $C_{g}^{l, i_{1} \ldots i_{\mu}}$. (Note that by weight considerations, $0 \leq \operatorname{link}(l) \leq 3$.) Let $B$ be the maximum value of $\operatorname{link}(l)$, $l \in \bar{L}_{\text {not.same }}$, and denote by $\bar{L}_{\text {not.same }}^{B} \subset \bar{L}_{\text {not.same }}$ the corresponding index set. We will then prove our claim for the tensor fields indexed in $\bar{L}_{\text {not.same }}^{B}$. By repeating this step at most four times, we will derive our third claim.

Consider silly $\left[L_{g}\left(\Omega_{1}, \ldots, \Omega_{p}, \phi_{1}, \ldots, \phi_{u}, v_{a b}\right)\right]=0$. Pick out the sublinear combination silly ${ }_{*}\left[L_{g}\left(\Omega_{1}, \ldots, \Omega_{p}, \phi_{1}, \ldots, \phi_{u}, v_{a b}\right)\right]=0$ with $\mu-M-2$ internal contractions, and with extra $M+2$ derivatives on the factors $T^{j}, T^{l}$ against which the two indices of the factor $v_{a b}$ contract, and with $M+2+B$ particular contractions between the factors $T^{j}, T^{l}$. This sublinear combination must vanish separately,

$$
\text { silly }_{*}\left[L_{g}\left(\Omega_{1}, \ldots, \Omega_{p}, \phi_{1}, \ldots, \phi_{u}, v_{a b}\right)\right]=0 .
$$

Moreover, we observe by following the "inverse integration by parts" and the silly divergence formula obtained from $\int_{M^{n}} Z_{g}\left(v_{a b}\right) d V_{g}=0$, that the left-hand side of the above can be described as follows:

For each $C_{g}^{l, i_{1} \ldots i_{\mu}}, l \in \bar{L}_{\text {not.same }}^{B}$, we denote by $\tilde{C}_{g}^{l}\left(v_{a b}\right)$ the complete contraction that arises by replacing the factor $T=\nabla_{(\text {free }) \ldots(\text { free })}^{(M)} R_{(\text {free }) j(\text { free }) l}$ by $\nabla_{(\text {free }) \ldots(\text { free })}^{(M+2)} v_{j l}$, and then replacing each free index that does not belong to the factor $T$ by an internal contraction. We then denote by $\hat{C}_{g}^{l}\left(v_{a b}\right)$ the complete contraction that arises from $\tilde{C}_{g}^{l}\left(v_{a b}\right)$ by hitting the factor $T^{j}$ (against which the index ${ }_{j}$ in $v_{j l}$ contracts) by $(M+2)$ derivative indices $\nabla_{t_{1}}, \ldots, \nabla_{t_{M+2}}$ and hitting the factor $T^{l}$ (against which the index $l$ in $v_{j l}$ contracts) by derivatives $\nabla^{t_{1}}, \ldots, \nabla^{t_{M+2}} .{ }^{82}$ It follows that

$$
(0=) \operatorname{silly}_{*}\left[L_{g}\left(\Omega_{1}, \ldots, \Omega_{p}, \phi_{1}, \ldots, \phi_{u}, v_{a b}\right)\right]=\sum_{\bar{L}_{\text {not.same }}^{B}} a_{l} 2^{M+1}\left[\hat{C}_{g}^{l}\left(v_{a b}\right)\right] .
$$

Now, to derive our claim, we introduce a formal operation $\mathrm{Op}[\ldots]$ which acts on the terms above by applying $\mathrm{Sub}_{v}$ to each of the $\mu-M-2$ internal contractions, ${ }^{83}$ erasing $M+2$ particular contractions between the factors $T^{j}, T^{l}$ and then replacing the factor $v_{j l}$ by $\nabla_{r_{1} \ldots r_{M}}^{(m)} R_{i j k l} \nabla^{r_{1}} v \ldots \nabla^{r_{M}} v \nabla^{i} v \nabla^{k} v$. This operation produces a new true equation; after we divide this new true equation by $2^{M+1}$, we derive our claim.

Note on the derivation of (3-9), (3-10). The equations can be derived by a straightforward modification of the ideas above: The only extra feature we add is that in

${ }^{82}$ These derivatives contract against the indices $\nabla_{t_{1}}, \ldots, \nabla_{t_{M+2}}$ that have hit $T^{j}$.

${ }^{83}$ See the Appendix of [A 2012] for the definition of this operation. 
the silly divergence formula we must pick out the terms for which (both/one of the) indices $_{j, l}$ in $v_{j l}$ contract against a special index in a factor $S_{*} \nabla^{(M+2)} R_{a b c d} \nabla^{a} \tilde{\phi}_{h}$. This linear combination will vanish, modulo terms where one/none of the indices ${ }_{j}, l$ in $v_{j l}$ contract against a special index in the factor $S_{*} R_{i j k l}$ : This follows by the same argument that is used in [A 2010] to derive that Lemma 3.1 in [A 2010] implies Proposition 1.1 in Case I: We first replace the factor $v_{j l}$ by an expression $y_{(j} y_{l)}$. We then just replace both/one of the expressions $\nabla_{i} \tilde{\phi}_{h}, y_{j}$ by $g_{i j}$ and apply Ricto $\Omega$ twice/once. ${ }^{84}$ The only terms that survive this true equation are the ones indexed in $\bar{L}_{\text {not.same }}$, for which the expression(s) $S_{*} \nabla_{r_{1} \ldots r_{v}}^{(v)} R_{i j k l} \nabla^{i} \tilde{\phi}_{h} \nabla^{k} y$ are replaced by $\nabla_{r_{1} \ldots r_{v} j l}^{(v+2)} Y_{f}$. We then proceed as above, deriving that the sublinear combination of terms indexed in $\bar{L}_{\text {not.same }}$ must vanish, after we replace two/one expressions $S_{*} \nabla_{r_{1} \ldots r_{v}}^{(v)} R_{i j k l} \nabla^{i} \tilde{\phi}_{h} \nabla^{k} y$ by $\nabla_{r_{1} \ldots r_{\nu} j l}^{(v+2)} Y_{f}$. Then, repeating the permutations applied to any factors $\nabla_{r_{1} \ldots r_{v} j l}^{(v+2)} Y_{f}$, to $S_{*} \nabla_{r_{1} \ldots r_{v}}^{(v)} R_{i j k l} \nabla^{i} \tilde{\phi}_{h} \nabla^{k} y$ we derive our claim.

3B. The remaining cases of Proposition 1.1 in Case III. We recall that there are remaining cases only when $\sigma=3$. In that case we have the remaining cases when $p=3$ and $n-2 u-2 \mu \leq 2$, or when $p=2, \sigma_{2}=1$ and $n=2 u+2 \mu$.

The case $p=3$. Let us start with the subcase $n-2 u-2 \mu=0$. In this case, all tensor fields in (1-7) will be in the form

$$
\begin{aligned}
\operatorname{pcontr}( & \nabla_{i_{1} \ldots i_{a} j_{1} \ldots j_{b}}^{(A)} \Omega_{1} \otimes \nabla_{i_{a+1} \ldots i_{a+a^{\prime}} j_{b+1} \ldots j_{b+b^{\prime}}}^{(B)} \Omega_{2} \\
& \left.\otimes \nabla_{i_{a+a^{\prime}+1}^{(C)} \ldots i_{a+a^{\prime}+a^{\prime \prime}} j_{b+b^{\prime}+1} \ldots j_{b+b^{\prime}+b^{\prime \prime}}} \Omega_{3} \otimes \nabla^{j_{x_{1}}} \phi_{1} \cdots \otimes \nabla^{x_{j+j^{\prime}+j^{\prime \prime}}} \phi_{u}\right),
\end{aligned}
$$

where we make the following conventions: Each of the indices $i_{f}$ is free; also, each of the indices $j_{f}$ contracts against some factor $\nabla \phi_{h}$, and also $A, B, C \geq 2$.

Thus, we observe that in this subcase $\mu$ is also the maximum rank among the tensor fields appearing in (1-7). Now, assume that the $\mu$-tensor fields in (1-7) of maximal refined double character have $a=\alpha, a^{\prime}=\alpha^{\prime}, a^{\prime \prime}=\alpha^{\prime \prime}$. With no loss of generality (only up to renaming the factors $\Omega_{1}, \Omega_{2}, \Omega_{3}, \phi_{1}, \ldots, \phi_{u}$ ) we may assume that $\alpha \geq \alpha^{\prime} \geq \alpha^{\prime \prime}$ and that only the functions $\nabla \phi_{1}, \ldots, \nabla \phi_{u_{1}}$ contract against

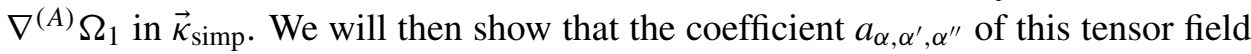
must be zero. This will prove Proposition 1.1 in this subcase.

We prove that $a_{\alpha, \alpha^{\prime}, \alpha^{\prime \prime}}=0$ by considering the global equation $\int Z_{g} d V_{g}=0$ and considering the silly divergence formula silly $\left[Z_{g}\right]=0$. We then consider the sublinear combination silly ${ }_{+}\left[Z_{g}\right]$ consisting of terms with $\alpha^{\prime}, \alpha^{\prime \prime}$ internal contractions in the factors $\nabla^{(D)} \Omega_{2}, \nabla^{(E)} \Omega_{3}$, with $\alpha$ particular contractions between those factors and with all factors $\nabla \phi_{h}$ that contracted against $\nabla^{(A)} \Omega_{1}$ in $\vec{\kappa}_{\text {simp }}$ being replaced by $\Delta \phi_{h}$, while all factors $\nabla \phi_{h}$ that contracted against $\nabla^{(B)} \Omega_{2}, \nabla^{(C)} \Omega_{3}$ still do so.

\footnotetext{
${ }^{84}$ Recall that this operation has been defined in the Appendix in [A 2012] and produces a true equation.
} 
We easily observe that silly ${ }_{+}\left[Z_{g}\right]=0$, and furthermore silly ${ }_{+}\left[Z_{g}\right]$ consists of the complete contraction

$$
\begin{aligned}
& \operatorname{contr}\left(\Omega_{1} \otimes \nabla^{f_{1} \ldots f_{\alpha}}{ }_{j_{b+1} \ldots j_{b+b^{\prime}}} \Delta^{\alpha^{\prime}} \Omega_{2} \otimes \nabla_{f_{1} \ldots f_{\alpha} j_{b+b^{\prime}+1} \ldots j_{b+b^{\prime}+b^{\prime \prime}}} \Delta^{\alpha^{\prime \prime}} \Omega_{3}\right.
\end{aligned}
$$

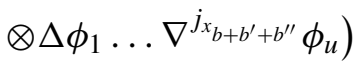

times the constant $(-1)^{u_{1}} 2^{\alpha} a_{\alpha, \alpha^{\prime}, \alpha^{\prime \prime}}$. Thus, we derive that $a_{\alpha, \alpha^{\prime}, \alpha^{\prime \prime}}=0$.

The second subcase. We now consider the setting where $\sigma=p=3, n-2 u-2 \mu=$ 2. In this setting, the maximum rank of the tensor fields appearing in (1-7) is $\mu+1$. In this case, all ( $\mu+1)$-tensor fields in (1-7) will be in the form (3-11) (with $\alpha+\alpha^{\prime}+\alpha^{\prime \prime}=\mu+1$, while all the $\mu$-tensor fields will be in the form (3-11) but with $\alpha+\alpha^{\prime}+\alpha^{\prime \prime}=\mu$, and with one particular contraction ${ }_{c},{ }^{c}$ between two of the factors $\nabla^{(A)} \Omega_{1}, \nabla^{(B)} \Omega_{2}, \nabla^{(C)} \Omega_{3}$.

Now, if both the indices ${ }_{c},{ }^{c}$ described above are removable, we can explicitly express $C_{g}^{l, i_{1} \ldots i_{\mu}}$ as an $X$ div of an acceptable $(\mu+1)$-tensor field. Therefore, we are reduced to showing our claim in this setting where for each $\mu$-tensor field in (1-7) at least one of the indices ${ }_{c},{ }^{c}$ is not removable. Now, let $z \in Z_{\operatorname{Max}}$ stand for one of the index sets for which the sublinear combination $\sum_{l \in L^{z}} a_{l} C_{g}^{l, i_{1} \ldots i_{\mu}}$ in (1-7) indexes tensor fields of maximal refined double character. We assume with no loss of generality that for each $l \in L^{z}$ the factors $\nabla^{(A)} \Omega_{1}, \nabla^{(B)} \Omega_{2}, \nabla^{(C)} \Omega_{3}$ have $\alpha \geq \alpha^{\prime} \geq \alpha^{\prime \prime}$ free indices respectively. ${ }^{85}$ Therefore, the tensor fields indexed in $L^{z}$ can be in one of the two forms

$$
\begin{aligned}
\operatorname{pcontr} & \nabla^{c} \nabla_{i_{1} \ldots i_{\alpha} j_{1} \ldots j_{b}}^{(A)} \Omega_{1} \otimes \nabla_{i_{\alpha+1} \ldots i_{\alpha+\alpha^{\prime}} j_{b+1} \ldots j_{b+b^{\prime}}}^{(B)} \Omega_{2} \\
& \left.\otimes \nabla_{c i_{\alpha+\alpha^{\prime}+1} \ldots i_{\alpha+\alpha^{\prime}+\alpha^{\prime \prime}} j_{b+b^{\prime}+1} \ldots j_{b+b^{\prime}+b^{\prime \prime}}}^{(2)} \Omega_{3} \otimes \nabla^{j_{x_{1}}} \phi_{1} \cdots \otimes \nabla^{x_{j+j^{\prime}+j^{\prime \prime}}} \phi_{u}\right),
\end{aligned}
$$

or

$$
\begin{aligned}
\operatorname{pcontr} & \nabla_{i_{1} \ldots i_{\alpha} j_{1} \ldots j_{b}}^{(A)} \Omega_{1} \otimes \nabla^{c} \nabla_{i_{\alpha+1} \ldots i_{\alpha+\alpha^{\prime}} j_{b+1} \ldots j_{b+b^{\prime}}}^{(B)} \Omega_{2} \\
& \left.\otimes \nabla_{c i_{\alpha+\alpha^{\prime}+1} \ldots i_{\alpha+\alpha^{\prime}+\alpha^{\prime \prime}} j_{b+b^{\prime}+1} \ldots j_{b+b^{\prime}+b^{\prime \prime}}}^{(2)} \Omega_{3} \otimes \nabla^{j_{x_{1}}} \phi_{1} \cdots \otimes \nabla^{x_{j+j^{\prime}+j^{\prime \prime}}} \phi_{u}\right),
\end{aligned}
$$

where $A, B \geq 3$.

Now, by "manually subtracting" $X$ divs from these $\mu$-tensor fields, we can assume without loss of generality that the tensor fields indexed in our chosen $L^{z}$ are in the form (3-14).

With that extra assumption, we can show that the coefficient of the tensor field (3-14) is zero. We see this by considering the (global) equation $\int_{M} Z_{g} d V_{g}=0$ and using the silly divergence formula silly $\left[Z_{g}\right]=0$ (which arises by integrations by parts with respect to the factor $\nabla^{(A)} \Omega_{1}$ ). Picking out the sublinear combination

${ }^{85}$ Recall that by our hypothesis $\alpha^{\prime} \geq 2$. 
silly ${ }_{+}\left[Z_{g}\right]$ which consists of the complete contraction

$$
\begin{aligned}
& \operatorname{contr}\left(\Omega_{1} \otimes \nabla_{j_{b+1} \ldots j_{b+b^{\prime}}}^{c f_{1} \ldots f_{\alpha}} \Delta^{\alpha^{\prime}} \Omega_{2} \otimes \nabla_{c f_{1} \ldots f_{\alpha} j_{b+b^{\prime}+1} \ldots j_{b+b^{\prime}+b^{\prime \prime}}} \Delta^{\alpha^{\prime \prime}} \Omega_{3}\right.
\end{aligned}
$$

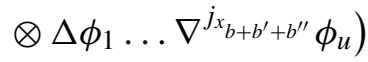

(notice that silly ${ }_{+}\left[Z_{g}\right]=0$ ), we derive that the coefficient of (3-14) must vanish. Thus, we have shown our claim in this second subcase also.

The case $p=2, \sigma_{2}=1$. Recall that in this case we fall under the special case when $n=2 u+2 \mu$. In this setting, we will have that in each index set $L^{z}, z \in Z_{\text {Max }}^{\prime}$, (see the statement of Lemma 3.5 in [A 2010]) there is a unique $\mu$-tensor field of maximal refined double character in (1-7), where the two indices ${ }_{k}, l$ in the factor $S_{*} \nabla^{(v)} R_{i j k l}$ will be contracting against one of the factors $\nabla^{(A)} \Omega_{1}, \nabla^{(B)} \Omega_{2}$ (without loss of generality we may assume that they are contracting against different factors). But now, recall that since we are considering Case $A$ of Lemma 3.5 in [A 2010], one of the factors $\nabla^{(A)} \Omega_{1}, \nabla^{(B)} \Omega_{2}$ will have at least two free indices. Hence, in at least one of the factors $\nabla^{(A)} \Omega_{1}, \nabla^{(B)} \Omega_{2}$, the index ${ }^{k},{ }^{l}$ is removable (meaning that it can be erased, and we will be left with an acceptable tensor field). We denote by $C_{g}^{l, i_{1} \ldots i_{\mu} i_{\mu+1}}$ the tensor field that arises from $C_{g}^{l, i_{1} \ldots i_{\mu}}$ by erasing the aforementioned ${ }^{k},{ }^{l}$ and making ${ }_{k}$ or ${ }_{l}$ into a free index, we then observe that

$$
C_{g}^{l, i_{1} \ldots i_{\mu}}-X \operatorname{div}_{i_{\mu+1}} C_{g}^{l, i_{1} \ldots i_{\mu} i_{\mu+1}}=0
$$

(modulo complete contractions of length $\geq \sigma+u+1$ ). This completes the proof of our claim.

\section{Acknowledgement}

This work has absorbed the best part of the author's energy over many years. This research was partially conducted during the period the author served as a Clay Research Fellow, an MSRI postdoctoral fellow, a Clay Liftoff fellow and a Procter Fellow.

The author is immensely indebted to Charles Fefferman for devoting twelve long months to the meticulous proof-reading of the present paper. He also wishes to express his gratitude to the Mathematics Department of Princeton University for its support during his work on this project.

\section{References}

[A 2009] S. Alexakis, "On the decomposition of global conformal invariants, I", Ann. of Math. (2) 170:3 (2009), 1241-1306. MR 2011g:53063 Zbl 1190.53028

[A 2010] S. Alexakis, "The decomposition of global conformal invariants, IV: A proposition on local Riemannian invariants”, Adv. Math. 225:2 (2010), 515-597. MR 2012b:53049 Zbl 1198.53034 
[A 2011] S. Alexakis, "The decomposition of global conformal invariants: some technical proofs, I", Symmetry Integrability Geom. Methods Appl. 7 (2011), Paper 019. MR 2804577 Zbl 1218.53038

[A 2012] S. Alexakis, The decomposition of global conformal invariants, Annals of Mathematics Studies 182, Princeton University Press, 2012. MR 2918125 Zbl 06035836

[Bailey et al. 1994a] T. N. Bailey, M. G. Eastwood, and A. R. Gover, "Thomas's structure bundle for conformal, projective and related structures", Rocky Mountain J. Math. 24:4 (1994), 1191-1217. MR 96e:53016 Zbl 0828.53012

[Bailey et al. 1994b] T. N. Bailey, M. G. Eastwood, and C. R. Graham, "Invariant theory for conformal and CR geometry", Ann. of Math. (2) 139:3 (1994), 491-552. MR 95h:53016 Zbl 0814.53017

[Čap and Gover 2002] A. Čap and A. R. Gover, "Tractor calculi for parabolic geometries", Trans. Amer. Math. Soc. 354:4 (2002), 1511-1548. MR 2003j:53033 Zbl 0997.53016

[Čap and Gover 2003] A. Čap and A. R. Gover, "Standard tractors and the conformal ambient metric construction”, Ann. Global Anal. Geom. 24:3 (2003), 231-259. MR 2004g:53016 Zbl 1039.53021

[Cartan 1896] E. Cartan, "Sur la reduction a sa forme canonique de la structure d'un groupe de transformations fini et continu", Amer. J. Math. 18:1 (1896), 1-61. Reprinted as pp. 293-355 in his Oeuvres Complètes, Partie 1, Gauthier-Villars, Paris, 1952. MR 1505696 JFM 27.0288.01

[Deser and Schwimmer 1993] S. Deser and A. Schwimmer, "Geometric classification of conformal anomalies in arbitrary dimensions", Phys. Lett. B 309:3-4 (1993), 279-284. MR 94g:81195

[Fefferman 1976] C. L. Fefferman, "Monge-Ampère equations, the Bergman kernel, and geometry of pseudoconvex domains", Ann. of Math. (2) 103:2 (1976), 395-416. Correction in 104:2 (1976), 393-394. MR 0407320 (53 \#11097a) Zbl 0322.32012

[Fefferman and Graham 1985] C. Fefferman and C. R. Graham, "Conformal invariants", pp. 95-116 in Élie Cartan et les mathématiques d'aujourd'hui (Lyon, 1984), Astérisque, 1985. Numéro hors série. MR 87g:53060 Zbl 0602.53007

[Graham and Hirachi 2008] C. R. Graham and K. Hirachi, "Inhomogeneous ambient metrics", pp. 403-420 in Symmetries and overdetermined systems of partial differential equations (Minneapolis, MN, 2006), edited by M. Eastwood and W. Miller, Jr., IMA Vol. Math. Appl. 144, Springer, New York, 2008. MR 2009f:53012 Zbl 1148.53023

[Hirachi 2000] K. Hirachi, "Construction of boundary invariants and the logarithmic singularity of the Bergman kernel”, Ann. of Math. (2) 151:1 (2000), 151-191. MR 2001f:32003 Zbl 0954.32002

[Thomas 1934] T. Y. Thomas, The differential invariants of generalized spaces, Cambridge University Press, 1934. JFM 60.0363.02

[Weyl 1939] H. Weyl, The classical groups: Their invariants and representations, Princeton University Press, 1939. MR 0000255 (1,42c) Zbl 0020.20601

Received December 21, 2009.

\section{SPyros AlEXAKIS}

DEPARTMENT OF MATHEMATICS

UNIVERSITY OF TORONTO

$40 \mathrm{ST}$. GEORGE ST

TORONTO M5S 2E4

CANADA

alexakis@math.toronto.edu 


\title{
PACIFIC JOURNAL OF MATHEMATICS
}

\author{
http://pacificmath.org
}

Founded in 1951 by E. F. Beckenbach (1906-1982) and F. Wolf (1904-1989)

\section{EDITORS}

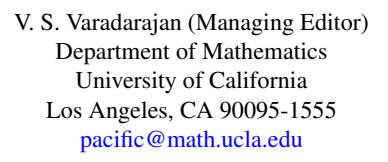

Don Blasius

Department of Mathematics University of California

Los Angeles, CA 90095-1555

blasius@math.ucla.edu

Robert Finn

Department of Mathematics

Stanford University

Stanford, CA 94305-2125

finn@math.stanford.edu

Alexander Merkurjev

Department of Mathematics

University of California

Los Angeles, CA 90095-1555

merkurev@math.ucla.edu

\author{
Vyjayanthi Chari \\ Department of Mathematics \\ University of California \\ Riverside, CA 92521-0135 \\ chari@math.ucr.edu \\ Kefeng Liu \\ Department of Mathematics \\ University of California \\ Los Angeles, CA 90095-1555 \\ liu@math.ucla.edu \\ Sorin Popa \\ Department of Mathematics \\ University of California \\ Los Angeles, CA 90095-1555 \\ popa@math.ucla.edu \\ Paul Yang \\ Department of Mathematics \\ Princeton University \\ Princeton NJ 08544-1000 \\ yang@math.princeton.edu
}

\section{PRODUCTION}

Silvio Levy, Scientific Editor, pacific@math.berkeley.edu

\section{SUPPORTING INSTITUTIONS}

ACADEMIA SINICA, TAIPEI

CALIFORNIA INST. OF TECHNOLOGY

INST. DE MATEMÁTICA PURA E APLICADA

KEIO UNIVERSITY

MATH. SCIENCES RESEARCH INSTITUTE

NEW MEXICO STATE UNIV.

OREGON STATE UNIV.

\author{
STANFORD UNIVERSITY \\ UNIV. OF BRITISH COLUMBIA \\ UNIV. OF CALIFORNIA, BERKELEY \\ UNIV. OF CALIFORNIA, DAVIS \\ UNIV. OF CALIFORNIA, LOS ANGELES \\ UNIV. OF CALIFORNIA, RIVERSIDE \\ UNIV. OF CALIFORNIA, SAN DIEGO \\ UNIV. OF CALIF., SANTA BARBARA
}

\author{
Daryl Cooper \\ Department of Mathematics \\ University of California \\ Santa Barbara, CA 93106-3080 \\ cooper@math.ucsb.edu \\ Jiang-Hua Lu \\ Department of Mathematics \\ The University of Hong Kong \\ Pokfulam Rd., Hong Kong \\ jhlu@maths.hku.hk
}

\section{Jie Qing}

Department of Mathematics

University of California

Santa Cruz, CA 95064

qing@cats.ucsc.edu

These supporting institutions contribute to the cost of publication of this Journal, but they are not owners or publishers and have no responsibility for its contents or policies.

See inside back cover or pacificmath.org for submission instructions.

The subscription price for 2012 is US \$420/year for the electronic version, and \$485/year for print and electronic.

Subscriptions, requests for back issues from the last three years and changes of subscribers address should be sent to Pacific Journal of Mathematics, P.O. Box 4163, Berkeley, CA 94704-0163, U.S.A. Prior back issues are obtainable from Periodicals Service Company, 11 Main Street, Germantown, NY 12526-5635. The Pacific Journal of Mathematics is indexed by Mathematical Reviews, Zentralblatt MATH, PASCAL CNRS Index, Referativnyi Zhurnal, Current Mathematical Publications and the Science Citation Index.

The Pacific Journal of Mathematics (ISSN 0030-8730) at the University of California, c/o Department of Mathematics, 969 Evans Hall, Berkeley, CA 94720-3840, is published monthly except July and August. Periodical rate postage paid at Berkeley, CA 94704, and additional mailing offices. POSTMASTER: send address changes to Pacific Journal of Mathematics, P.O. Box 4163, Berkeley, CA 94704-0163.

PJM peer review and production are managed by EditFlow ${ }^{\circledR}$ from Mathematical Sciences Publishers.

\section{PUBLISHED BY}

\section{mathematical sciences publishers}

http://msp.org/

A NON-PROFIT CORPORATION

Typeset in LATEX 


\section{PACIFIC JOURNAL OF MATHEMATICS}

Volume $260 \quad$ No. $1 \quad$ November 2012

The decomposition of global conformal invariants: Some technical proofs II

SPYROS ALEXAKIS

On deformation quantizations of hypertoric varieties

GWYN BELLAMY and TOSHIRO KUWABARA

Almost factoriality of integral domains and Krull-like domains

GYU Whan CHANG, HWANKOO KIM and JUNG WOOK LIM

Singularities of free group character varieties

CARLOS FLORENTINO and SEAN LAWTON

Energy identity for the maps from a surface with tension field bounded 181 in $L^{p}$

LI JIAYU and ZHU XIANGRONG

Remarks on some isoperimetric properties of the $k-1$ flow

YU-CHU LIN and DONG-Ho TSAI

Demystifying a divisibility property of the Kostant partition function 215

KAROLA MÉSZÁros

Exceptional Lie algebras, SU(3), and Jordan pairs

PIERO TRUINI

Lower estimate of Milnor number and characterization of isolated homogeneous hypersurface singularities

Stephen S.-T. Yau and HuAiqing Zuo 